\title{
Cultural Property und Cultural Heritage. \\ Eine ethnologische Analyse internationaler \\ Konzeptionen im Vergleich.
}

\begin{abstract}
Hausarbeit
zur Erlangung des Magistergrades (M.A.)

an der Sozialwissenschaftlichen Fakultät der Georg-August Universität Göttingen
\end{abstract}

\author{
vorgelegt von \\ Frank-André Weigelt \\ zu Göttingen
}

Göttingen, der 03.07.2006 


\section{Vorwort}

Während der Ausarbeitung und Erstellung meiner Magisterarbeit kam mir der Gedanke, ein kleines Vorwort zu verfassen, auch wenn dies nicht unbedingt üblich für eine derartige Arbeit ist.

Als ich meine anfänglichen Ideen im Institutskollogium vorstellte, wurde ich darauf hingewiesen, dass in einer ethnologisch ausgerichteten Arbeit ja auch Beispiele sein sollten, die aus der "Froschperspektive“ bzw. methodologisch auf der Grundlage einer „bottom up“-Analyse erarbeitet wurden.

Im Verlauf meiner Arbeit wurde mir klar, dass ich diese Prämisse nicht wirklich erfüllen kann, obwohl ich drei verschieden gelagerte Beispiele eingearbeitet habe. Mein thematischer Schwerpunkt liegt größten Teils auf der Darstellung theoretischer Erörterungen, Paradigmen und wissenschaftlicher Abstraktionen zu kulturellem Eigentum (Cultural Property) und kulturellem Erbe (Cultural Heritage). Ich fragte mich also, wie ich mich rechtfertigen könnte, obwohl ich mir auch klar war und bin, dass dies nicht zwingend notwendig ist.

Ich denke, dass es bei Anwendung einer „Froschperspektive“ auf den Standpunkt des Betrachters ankommt, sozusagen auf den Primat der „Froschperspektive“. Indem ich einen theoretisch orientierten Schwerpunkt auf Basis von Literatur erarbeite bin ich sozusagen - Platons Höhlengleichnis folgend - bereits bei einem „Blick von Unten“, anders gesagt bei einer "Schau der Ideen“, auch wenn dieses „Schau“ meistens auf und in die Bücher war.

Abschließend bedanke ich mich für die sehr gute Betreuung durch meine Prüferlnnen, die immer ein offenes Ohr für meine Probleme und Anliegen hatten. Vor allem auch bei meinen KorrektorInnen, die in unermüdlichem Einsatz für eine verbesserte Grammatik und Rechtschreibung sorgten und mir bei formalen Angelegenheiten im Bereich der EDV gute Verwahrenshinweise gaben. Des weiteren bedanke ich mich bei meinen ins Fach enkulturierten Kommilitonen, die trotz Fußball WM die Zeit fanden, mich auf so manche fachspezifischen Schwierigkeiten und Probleme in meiner Arbeit zu verweisen.

Göttingen der 03.07.2006 


\section{Inhaltsverzeichnis}

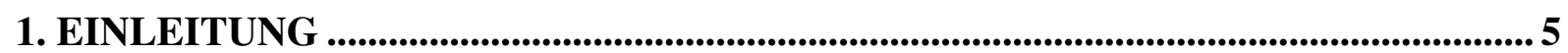

2. VON DEN SIEBEN WELTWUNDERN BIS ZUR UNESCO. EIN HISTORISCHER

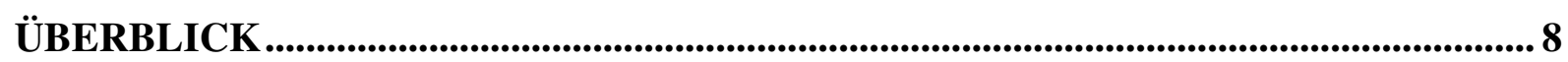

3. DIE UNESCO: ENTSTEHUNG, ZIELE, ORGANISATORISCHER AUFBAU........ 11

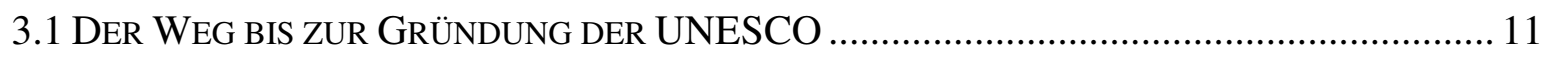

3.1.1 Die Zeit bis zu ersten Völkerbundversammlung 1920........................................ 12

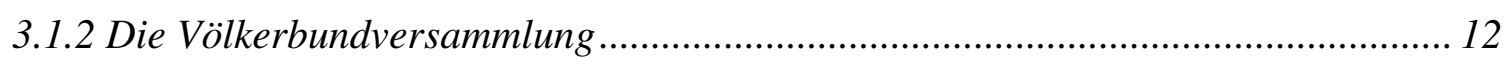

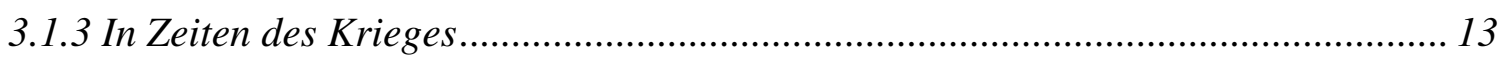

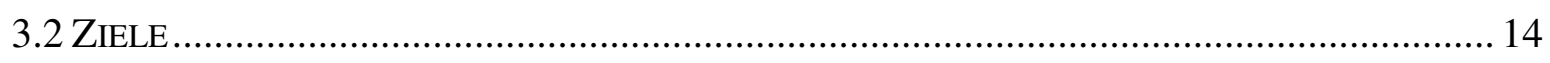

3.3 MitGLIEDSCHAFT UND ORGANISATORISCHER AUfBAU …............................................. 16

4. DIE BEDEUTUNG UND TRANSFORMATION DES KULTURBEGRIFFS IN DER

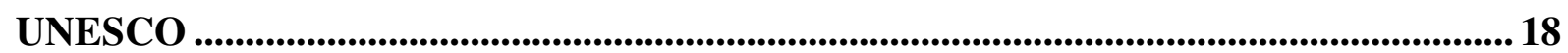

4.1 STATISCHER KULTURBEGRIFF VERSUS DYNAMISCHER KULTURBEGRIFF........................ 19

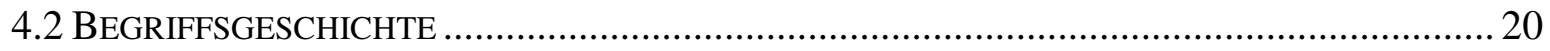

4.3 KULTUR IM ETHNOLOGISCH-KULTURWISSENSCHAFTLICHEN SINNE................................ 22

4.4 DAs KULTURKONZEPT DER UNESCO: VON DEN ANFÄNGEN BIS HEUTE .......................... 25

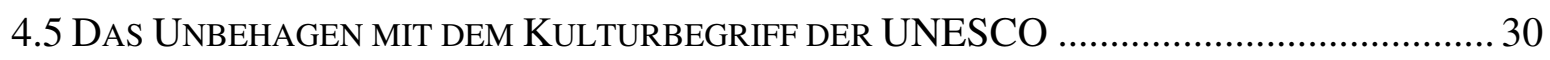

\section{DIE ANFÄNGE DER UNESCO-KONZEPTIONEN DES KULTURELLEN}

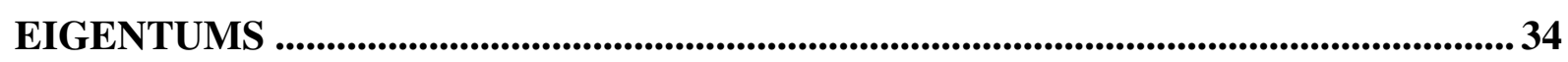

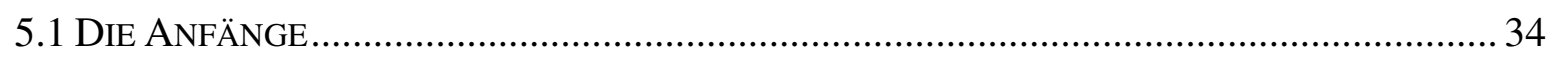

5.2 KONZEPTIONELLE ERWEITERUNGEN UND KULTURPOLITISCHER WANDEL....................... 35

5.2.1 Das Beispiel Abu Simble als materielles unbewegliches Eigentum ......................... 36

5.2.2 Maßnahmen gegen den internationalen Kunstraub ............................................. 37

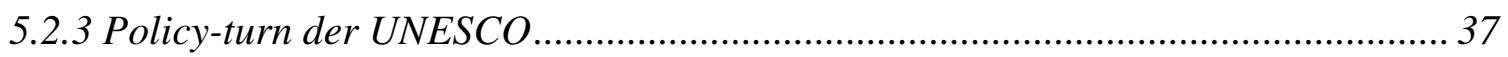

5.2.4 Die Entwicklungen seit der Welterbekonvention von 1972 ................................... 39

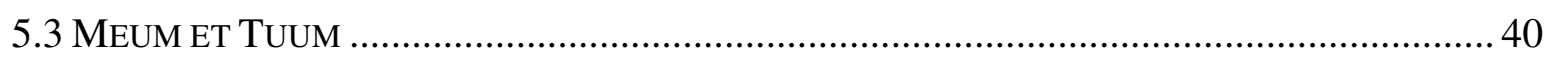

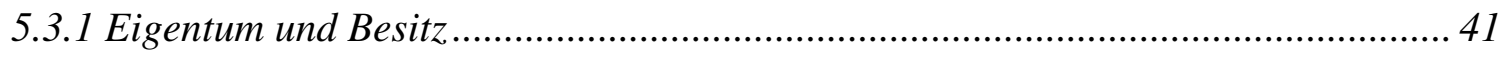

5.3.2 Ethnologische Perspektiven auf Eigentum und Besitz ......................................... 44

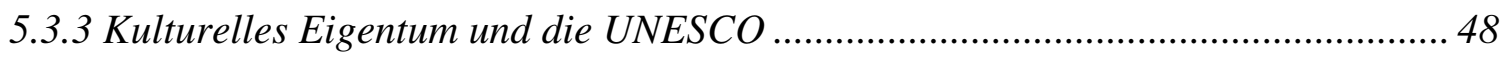


6.1 EIGENTUM ALS EURO-AMERIKANISCHES KONZEPT ..................................................... 59

6.2 MuLLICK VERSUS MULLICK: EIN FALL ÜBER MATERIELLES BEWEGLICHES EIGENTUM.. 60

6.3 EIGENTUM ALS ÖKONOMISCHER BEGRIFF. 61

6.4 KULTURELLES EIGENTUM UND DIE KATEGORIE DES IMMOBILEN/ GEISTIGEN .................. 62

6.5 DiE FUNDAMENTALE POLITIK HINTER KULTURELLEM EIGENTUM UND ERBE ...................63

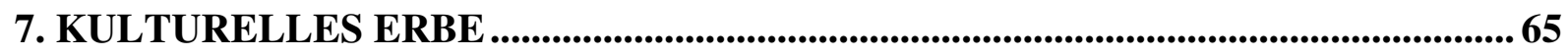

7.1 DiE ANFÄNGE DER IDEE EINES INTERNATIONALEN KULTUR- UND NATURERBES .............65

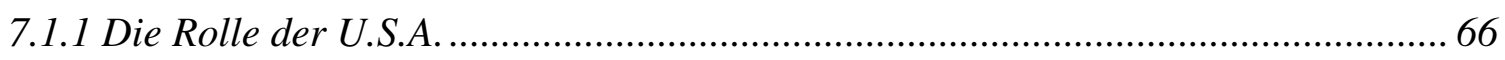

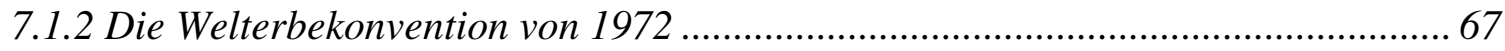

7.1.3 Die Generierung der Kategorie eines immateriellen Erbes.................................. 68

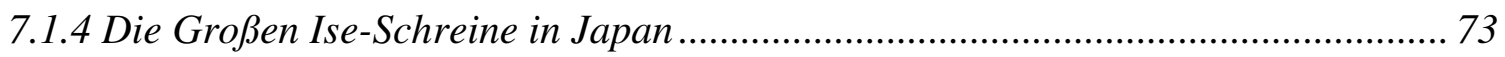

7.1.5 Weitere Projekte zur Erweiterung des Schutzhorizonts der UNESCO ................... 75

7.2 ERbE, ERBEN UND DIE TRAdITION. PhäNOMENOLOGISCHE SKIZZEN AUS

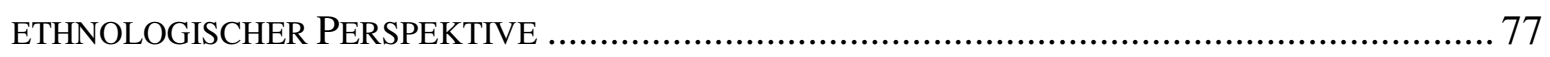

7.2.1 Eine kurze Etymologie der Begriffe Erbe und Tradition....................................... 78

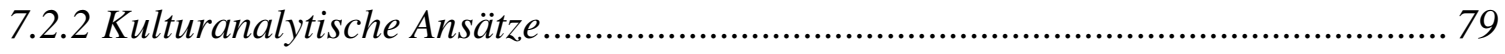

7.2.3 Kulturelles Erbe als soziale Konstruktion der Vergangenheit............................... 83

7.2.4 Kulturelles Erbe und die UNESCO-Konzeptionen.............................................. 87

8. KULTURELLES EIGENTUM UND KULTURELLES ERBE. EINE

KOMMENTIERTE ZUSAMMENFASSUNG. ..................................................................96

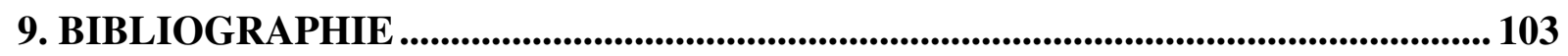

ABKÜRZUNGSVERZEICHNIS............................................................................... 132

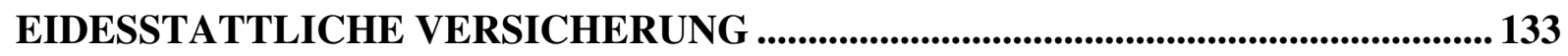




\section{Einleitung}

Das Interesse an Vergangenem, an überlieferten Werten und Traditionen, an Cultural Property und Cultural Heritage und eine damit verbundene Vielfalt an Maßnahmen zum Schutz, Restaurierung, Konservierung und einer ökonomischen Verwertbarkeit kultureller Elemente haben trotz eines oft proklamierten Bedeutungsverlustes bzw. Niedergangs der Tradition in einer posttraditionellen Gesellschaft (vgl. Giddens 1996) nach Ansicht vieler Autoren gegenwärtig nicht nur auf lokaler und nationaler Ebene Konjunktur, sondern auch auf internationaler Ebene.

Im Rahmen dieser Arbeit werde ich die internationalen Cultural Property und Cultural Heritage Konzeptionen der UNESCO näher betrachten. Zum einen wird es darum gehen, zu klären, warum und wieso es bei der UNESCO diese zwei unterschiedlichen Konzeptionen gibt und in welchem Zusammenhang bzw. historischen Kontext sie zu sehen sind. Ich stelle dabei die Frage, was Cultural Property im Gegensatz zu Cultural Heritage auf internationaler Ebene im Rahmen der UNESCO darstellt und warum es zu einem konzeptionellen Wandel bzw. einer Verschiebung konzeptioneller Prioritäten kam.

Des weiteren werde ich die einzelnen Begrifflichkeiten bzw. konzeptionellen Komponenten aus ethnologischer Perspektive näher betrachten und den UNESCOKonzeptionen diskutierend gegenüberstellen. Ausgehend von basissemantischen Inhalten der einzelnen Begriffe Culture, Property und Heritage und der adjektivischen Erweiterung des Property und Heritage-Begriffs um die qualitative Eigenschaft des cultural, erörtere ich theoretische Interpretationen und Definitionen der einzelnen Phänomene. Die Frage, die sich mir dabei stellt ist, wie sich die einzelnen Komponenten theoretisch, ausgehend von basissemantischen Inhalten, aus einer ethnologisch-kulturwissenschaftlichen Perspektive fassen lassen, in welchem weiteren $\mathrm{Zu}$ sammenhang sie zu sehen sind, und wie sich diese theoretischen Konstruktionen denen der UNESCO gegenüber abbilden bzw. welche Gemeinsamkeiten und Brüche erkennbar werden.

Ich werde, abgesehen vom Titel der Arbeit und der Einleitung, deutsche Übersetzungen statt englischer Termini verwenden, um vor allem einen besseren Lesefluss der Arbeit zu gewährleisten. Ich übersetze hierbei Property als Eigentum, Heritage als Erbe und Culture als Kultur. Die Begriffskonstellationen Cultural Property und Cultu- 
ral Heritage übersetze ich ins deutsche als kulturelles Eigentum und kulturelles Erbe. Mir ist bewusst, dass diese Form der Übersetzung nicht unbedingt auch äquivalente Sinngehalte der unterschiedlichen Termini mitträgt. Auf diese Problematik und weitere Übersetzungen englischer Termini (wie z.B. tangible, immovable oder Intellectual Property u.a.) gehe ich ausführlicher erst im späteren Verlauf der Arbeit ein.

Ein weiteres Anliegen dieser Arbeit ist es einen Beitrag zu leisten, um einer oft uneinheitlichen und nicht deutlich von einander abgegrenzten Verwendung der beiden Begriffskonstellationen Cultural Property und Cultural Heritage im Rahmen der aktuellen Diskussionen, kulturpolitischen Bestrebungen und Forschungsinteressen, theoretische Grundlagen und Abstraktionen für eine mögliche Differenzierung zur Verfügung zu stellen.

Um Antworten und Aussagen zu den aufgeworfenen Fragen zu erörtern und darzustellen, gliedere ich den Ablauf dieser Arbeit in acht Kapitel. Von der Einleitung ausgehend stelle ich einführend einen kurzen Überblick des bereits früh bezeugten Interesses der "Menschheit" an einem Cultural Property und Cultural Heritage dar und wie sich die Phänomene in groben Zügen bis zur Begründung der auf diesem Gebiet international bedeutsamsten Institution UNESCO wie ein Roter Faden durch die westliche Historie ziehen.

Im daran folgenden Teil erörtere ich in einer kurzen Zusammenfassung die Entstehung, Ziele und den organisatorischen Aufbau der UNESCO, um vorab ein grundlegendes Verständnis über und einen Einblick in diese Institution zu gewinnen.

Anschließend skizziere ich den Wandel und die Bedeutung des Kulturbegriffs der UNESCO für die Konzeptionen. Der Kulturbegriff kann in seiner Adjektivform als fundamentaler Bestandteil interpretiert werden, der die Begriffe Property und Heritage durch die qualitative Erweiterung des Kulturellen auf eine andere Ebene transformiert und damit ein vertiefendes Verständnis der Konzeptionen und des historischen Wandels vom konzeptionellen Fokus auf Property hin zur Hervorhebung des Heritage-Konzepts bei der UNESCO ermöglicht und auch die auf internationaler Ebene kulturpolitischen Veränderungen und Ambitionen der Staatengemeinschaft verdeutlicht. Ich behandle dabei ausschließlich das für den Rahmen dieser Arbeit als notwendig erachtete Wissen über das Phänomen Kultur.

Nach der Betrachtung des Kulturbegriffs der UNESCO und ethnologischer Perspektiven auf das Phänomen Kultur erarbeite ich dann die anfänglichen UNESCOKonzeptionen eines Cultural Property. Einleitend wird die historische Entwicklung 
und Genese der Konzeptionen dargestellt, um anschließend in einer analytischen Erörterung die begrifflichen Bestandteile aus ethnologischer Perspektive erklärend und im Kontrast der UNESCO-Konzeption zu diskutieren.

Im Anschluss hebe ich einige prägnante Punkte hervor, die für einen konzeptionellen Wandel von Cultural Property hin zu Cultural Heritage bei der UNESCO ausschlaggebend waren. Es soll dabei eine bessere Einsicht in die Beweggründe der Organisation für einen Wandel ermöglicht werden.

Im weiteren Ablauf gehe ich auf die UNESCO-Konzeptionen eines Cultural Heritage ein. Wie im Kapitel zu Cultural Property erörtere ich anfänglich die historische Entwicklung und Genese der Konzeptionen, um in einem weiteren Schritt aus ethnologischer Perspektive die jeweiligen Bestandteile der Begriffskonstellation darzustellen. Die erarbeiteten Fakten zur Analyse der Begrifflichkeiten stelle ich zum Abschluss der UNESCO-Konzeption gegenüber und diskutiere sie.

Im letzten und abschließenden Kapitel der Arbeit erfolgt die Zusammenfassung aller Vorangegangenen mit Kommentierung im Zusammenhang der Thematik als wichtig erachtete Punkte.

Bei der Erarbeitung, Darstellung und Analyse der Konzeptionen begrenze ich mich ausschließlich auf das für diese Arbeit als wichtig beurteilte Wissen. Ich kann im Rahmen dieser Arbeit keine umfassende Darstellung jedes einzelnen Aspektes erarbeiten, begründet durch die, meiner Meinung nach, uneinheitliche Quellenlage und Begriffsverwendung und -definition von Cultural Property und Cultural Heritage. Insgesamt standen ausreichend Primär- und Sekundärquellen zur Thematik zu Verfügung. Allein die Publikationen der UNESCO mussten auf Umwegen besorgt werden, d.h. alle Konventionen, Erklärungen und Empfehlungen waren nur über das Internet zu besorgen oder im UNESCO-Archiv der Staatsbibliothek zu Berlin. Die Bibliographie enthält nur tatsächlich eingesehene und verwendete Literatur bis auf eine Publikation von Benda-Beckmann und Wiber, die bis dato noch nicht veröffentlicht ist.

Vereinzelte Quellenkritik erörtere ich in der Arbeit selbst, damit der Leser einen direkten Bezug zu den Aussagen und den Zusammenhang, in dem die Quelle rezitiert wird, hat. 
„Die Vernunft dringt bis zum voraussetzungslosen Urbeginn von allem, um es anzurühren und dann wieder [...] herabzusteigen. Dabei nimmt sie überhaupt nichts sinnlich Wahrnehmbares zu Hilfe, sondern nur die Ideen selbst, schreitet so von Idee zu Idee und endet auch bei Ideen"

(Platon 1991:41)

\section{Von den Sieben Weltwundern bis zur UNESCO. Ein histo- rischer Überblick}

Das Interesse für Vergangenes, die Berufung auf überlieferte Werte und das Sinnen nach Schutz, Konservierung, Erhaltung und Erneuerung von bedeutsam kulturellen Errungenschaften ist seit der europäischen Antike ein mehr und minder vorhandenes Phänomen.

Bereits seit dieser Zeit bemühten sich Autoren um Reflexionen über Verfall, Zerstörung, Untergang und den Schutz von „kulturellem Eigentum“. Polybius von Athen schrieb im Jahre 146 v. Chr.:

"One may perhaps have some reason for amassing gold and silver; in fact, it would be impossible to attain universal dominion without appropriating these resources from other people, in order to weaken them. In the case of every other form of wealth, however, it is more glorious to leave it where it was, together with the envy it inspired, and to base our nation's glory, not on the abundance and beauty of its paintings and statues, but on its sober customs and noble sentiments. Moreover, I hope that future conquerors will learn from these thoughts not to plunder the cities subjugated by them, and not to make the misfortunes of other peoples the adornments of their own country" (Polybius zitiert in Merryman 2005:13; Übersetzung vgl. Merryman 2005:13).

Einige Jahre darauf schrieb Marcus Terentius Varro (116-72 v.Chr.) von den „septem opera in orbe terrae miranda“ (frei übersetzt: „von den sieben bewundernswerten Werken auf dem Erdkreis“; vgl. Lipp 2005: 19). Der Autor begründete damit als erster den Begriff der Sieben Weltwunder, jene Objekte also, die heute zweifellos auf der Welterbeliste der UNESCO ständen. Dies waren die Mauern von Babylon, die Statue des Zeus von Olympia, die hängenden Gärten von Babylon, der Koloss des Helios von Rhodos, die Pyramiden von Ägypten, das Mausoleum von Halikarnas und der Tempel der Artemis von Ephesos. Die erste vollständige Liste der Sieben Weltwun- 
der ist die „Anthologia Palatina“", ebenfalls aus dem zweiten Jahrhundert vor Christus. Philon von Byzanz, ein vermutlich spätantiker Autor, verfasste im weiteren Verlauf der Geschichte den so genannten „Reiseführer zu den Sieben Weltwundern“ (Lipp 2005: 19f; vgl. Brodersen 1997).

Einige Jahrhunderte später war das Interesse an vom Menschen geschaffenen Wunderwerken ebenso präsent wie in der Antike. Im Hochmittelalter (13. Jahrhundert) listet ein im Vatikan aufbewahrter Kodex alle in den bis dahin erschienenen Beschreibungen vorkommende Monumente auf und kommt zu einer Gesamtzahl von 30 Weltwundern (Lipp 2005:19f).

In der Renaissance ${ }^{2}$ besann man sich nicht nur alter antiker Werte, sondern auch wieder der Sieben Weltwunder. Meist wurde ein achtes Weltwunder durch einen Kommentator hinzugefügt. In unserer Zeit ist dies ein häufig auftretendes Phänomen in Bezug auf den Tourismus: z.B. der Mont-Saint-Michel in der Normandie/ Frankreich oder auch Angkor Wat in Siam Reap/ Kambodscha werden gerne als achtes Weltwunder gepriesen. Die erneute Beliebtheit der opera miranda zur Zeit der Renaissance bezeugt das Statut des Corpus Christi Colleges in Oxford aus dem Jahre 1517 n. Chr. Darin wurde festgehalten, dass die Dozenten nach dem Essen und Trinken noch zusammensitzen, miteinander singen oder über Dichtung, Geschichte und Weltwunder sprechen sollten (ebd.).

Als der Begriff der „culture“ ${ }^{\text {im }}$ im 18. Jahrhundert n. Chr. seine Wiederbelebung erfuhr, entstand auch die Vorstellung, „daß es so etwas wie eine kulturelle Erbschaft [bzw. „kulturellen Besitz"] jenseits der vererbbaren materiellen Werte des Einzelnen und ihrer Familien" gibt (Mayer 1982:40ff). Die Überlegung, kulturelle Artefakte als ein „nationales“ Erbe (Patrimoine) zu erklären, lässt sich für Frankreich zunächst 1790 durch Édouard Pommier festmachen. Die Überlegung entstand als Folge der radikalen Veränderungen, die die Französische Revolution ausgelöst hatte. Durch sie wur-

\footnotetext{
1 Der byzantinische Theologe Konstantinos Kephalas schuf um 900 n. Chr. in Byzanz eine nach Kategorien geordnete Anthologie, die vielfach erweitert wurde und heute unter dem Namen Anthologia Palatina bekannt ist (Köster 2005).

${ }^{2}$ Die Epoche der Renaissance (14.- 16. Jahrhundert n. Chr.) hat ihren Namen dem Umstand zu verdanken, das ihr wesentliches Charakteristikum die „Wiedergeburt“ des antiken Geistes war. Es ist ebenso das Zeitalter des Humanismus. Diese „Wiedergeburt" manifestierte sich darin, dass zahlreiche Elemente des Gedankenguts der Antike neu entdeckt und belebt wurden (Schriften, Baudenkmäler, Skulpturen, Philosophen, etc.; Kinder 1998:213).

${ }^{3}$ In Kapitel 4 wird näher auf die Entstehung, Bedeutung und Wandlung des Begriffs im Zusammenhang mit den Internationalen Konzeptionen des Cultural Property und Cultural Heritage eingegangen.
} 
den zahlreiche Denkmäler zerstört oder schwer beschädigt (Csáky\&Sommer 2005:1).

Hans Mayer (1982:18f) bemerkt für Deutschland, dass diese Tendenz „ziemlich genau zwischen 1773 und 1876“ entstanden sei. „Viele Zeitalter und Generationen [hätten] das Überlieferte und Hergebrachte durchaus nicht für erhaltenswert [erachtet]“. Erst auf der materiellen Basis des Bürgertums des 18. und 19. Jahrhunderts bildete sich ein ideologisches Konzept heraus, dem jene Überlieferungen als nationales Erbe galt, die zum schuldenfreien und wertbeständigen Besitz erklärt und kulturell vereinnahmt werden konnten, z.B. religiöse Gebäude, Wohlfahrts- und Bildungseinrichtungen, Werke der Kunst und der Wissenschaft (ebd.) ${ }^{4}$. Nietzsche (1937:266f) kommentierte die Begebenheiten jener Zeit wie folgt: „Vergangenheit als Erbe [sei] was man jetzt mit Vorliebe als den eigentlichen historischen Sinn bezeichnet“" (vgl. Bodner\&Sohm 2005: 12f).

Seit der ersten Erwähnung der Sieben Weltwunder, zwischenzeitlich 30 bewundernswerten Werken und den heutigen 754 (UNESCO Welterbeliste 2005), die durch die Welterbekonvention von 1972 in die Liste des Welterbes eingegangnen sind, liegt eine Zeitspanne von der Antike bis zur Gegenwart, liegt der Weg von der staunenden Bewunderung vergangener Größe zur Institutionalisierung der Erhaltungsbemühungen um das überlieferte Kultur- und Naturerbe der Welt.

Das Verständnis von Kultur, von kulturellen Entwicklungen und von der Bedeutung des kulturellen Erbes und des kulturellen Eigentums für die Menschheit hat sich im Laufe der Geschichte verändert. Es hat sich verändert, wie sich die Gesellschaften selbst verändert haben. Ebenso hat sich der Kulturbegriff in den Kulturwissenschaften und von einer statischen zu einer dynamischen Abstraktion gewandelt und wird heute auch von der UNESCO als eine dynamische Kategorie begriffen (s. Kapitel 4). Folglich sind auch die Kategorien des kulturellen Erbes, kulturellen Besitzes und die Konzeptionen ihres Schutzes Wandlungen unterworfen (vgl. Albert 2002:29).

In den folgenden Kapiteln stelle ich die anfänglichen Bemühungen der internationalen Staatengemeinschaft dar, hauptsächlich durch Schaffung der UNESCO ein internationales Instrument zum Schutze von kulturellem Eigentum und schließlich kulturellem Erbe zu etablieren.

\footnotetext{
${ }^{4}$ Bis ins 20. Jahrhundert waren dies auch die schützenswerten Kategorien durch die UNESCO-Konzeptionen, die vor allem kulturelles Eigentum als Schutzkategorie ausformulierten (s.u.).
} 


\section{Die UNESCO: Entstehung, Ziele, organisatorischer Auf- bau}

Die UNESCO ist in heutiger Zeit die wichtigste internationale Institution, wenn es um Fragen nach Schutz, Konservierung, Erhaltung und Erneuerung von bedeutsamen kulturellen Elementen geht. Die Einrichtung übernimmt in der Ausarbeitung von internationalen Rechtinstrumenten, wie völkerrechtlich bindenden Übereinkommen (Conventions), Empfehlungen (Recommendations) und Erklärungen (Declarations), die führende Rolle in der Welt, um einen Schutz von kulturellem Eigentum und mittlerweile vor allem von kulturellem Erbe zu bewirken.

\subsection{Der Weg bis zur Gründung der UNESCO}

Die United Nations Educational Scientific and Cultural Organization (Organisation der Vereinten Nationen für Erziehung, Wissenschaft, und Kultur), kurz UNESCO, wurde im gröberen Rahmen der 1945 in San Franzisko errichteten Organisation der United Nations (Vereinten Nationen ;kurz VN bzw. engl. UN) begründet (Menzel 1957:1f; vgl. Hüfner 1996).

Die Gründung der UNESCO war das Ergebnis einer längeren Entwicklung. Diese ging vor allem von privaten Vorschlägen aus und führte über die Bildung von zunächst ebenfalls privaten, später dann zwischenstaatlichen Vereinigungen für bestimmte Teilbezirke des kulturellen Lebens und den Bestrebungen des Völkerbundes zu der Notwendigkeit einer internationalen Zusammenarbeit auf den „weiten Gefilden“ der Erziehung und Kultur (Menzel 1957:1f). 


\subsubsection{Die Zeit bis zu ersten Völkerbundversammlung 1920}

Bereits vor dem Ersten Weltkrieg sind zahlreiche Vorschläge für bestimmte organisatorische Formen einer internationalen Zusammenarbeit auf dem Gebiet des Erziehungswesens und der Kultur gemacht worden. Die in den einzelnen Staaten gesammelten Erfahrungen sollten der Allgemeinheit zugänglich gemacht werden und gegenseitige Hilfsleistungen ermöglichen (Menzel 1957:1f).

Auch nach Beendigung des Ersten Weltkriegs hat es an Vorschlägen von fachmännischer Seite nicht gemangelt: z.B. die Anregungen des Schweizers Zollinger zur Bildung eines „internationalen Büros für Erziehungsfragen“ oder des Amerikaners Luckeny zur Errichtung eines International Education Research Council and World Bureau of Education. Doch diesen Versuchen war ebenso wenig Erfolg beschieden wie dem Vorschlag der Union des Associations Internationals, die den Plan einer internationalen Universität in sich schloss (ebd.).

Kennzeichnend für eine Behutsamkeit und Zurückhaltung, mit der die Staaten derartige Projekte zu behandeln pflegten, ist die Tatsache, dass die bei den Friedensverhandlungen 1919/20 eingereichten Denkschriften über die Bildung einer Internationalen Organisation für eine Zusammenarbeit der Nationen auf dem Gebiet der Kultur und Erziehung keine Berücksichtigung fanden (ebd.).

\subsubsection{Die Völkerbundversammlung}

Die erste Völkerbundversammlung (18.12.1920) fasste den Beschluss, dem Völkerbundrat zu empfehlen, „an den Bemühungen um die Verwirklichung einer internationalen Organisation der geistigen Arbeiter im Rahmen des Möglichen teilzunehmen“ (Menzel 1957:1). Im September 1921 legte dann der französische Völkerbunddelegierte Léon Burgeois den Entwurf einer solchen Organisation vor. Dieser unter dem Vorsitz des französischen Philosophen Henri Bergson arbeitende Ausschuss wies die zu jener Zeit hervorstechende Eigentümlichkeit auf, dass seine Mitglieder (u.a. Marie Curie, Albert Einstein) unabhängig von ihrer Staatsangehörigkeit berufen wurden und somit nicht als Staatsvertreter fungierten (ebd. 2f).

Die Völkerbundsversammlung etablierte auf einen französischen Vorschlag (23.09.1924) hin das Institut für geistige Zusammenarbeit in Paris. Die Gründung 
erwies sich für eine systematische Arbeitsplanung als glücklich. Es wurden u.a. Maßnahmen zur Förderung des Gedankenaustausches zwischen Universitäten, die Zusammenarbeit auf dem Gebiet des Bibliothekswesens und des Schutzes von Kunstwerken, des Urheberrechts, des Rundfunkwesens und Schulfilms erarbeitet (Menzel 1957:2f).

Die erzielten Fortschritte gaben im Jahre 1938 Anlass, die Ziele und Aufgabenbereiche des Instituts auf internationaler Ebene zu erweitern. Das entsprechende Abkommen wurde im selben Jahr von 45 Staaten unterzeichnet. Der Zweite Weltkrieg setzte jedoch der auf dieser erweiterten Grundlage begonnenen Arbeit ein Ende (ebd.)

\subsubsection{In Zeiten des Krieges}

Während sich Europa im Krieg befand, kam dem amerikanischen Kontinent als Wegbereiter des UNESCO-Gedankens eine besondere Bedeutung zu. Es wurden mehrere Ausschüsse gebildet, die zum Ziel hatten, die inneramerikanische Zusammenarbeit zu stärken. Die Konferenz von Havanna (1941) führte zur Bildung eines entsprechenden interamerikanischen Ausschusses und zur Planung einer Zentrale für geistige Zusammenarbeit (Menzel 1957:2f).

In den letzten Kriegsjahren des Zweiten Weltkriegs trafen sich in Europa die alliierten Bildungsminister in London, um die Lage des Erziehungswesens in den von den Achsenmächten besetzten Gebieten zu beraten. Es wurden in Übereinstimmung mit der amerikanischen Initiative Vorschläge zur Unterstützung der aus besetzten Gebieten stammenden Gastarbeiter, Kunst- und Denkmalschutz im Kriege, Gedanken- und Erfahrungsaustausch auf kulturellem und erzieherischem Gebiet der Nachkriegszeit und Maßnahmen zur Wiederherstellung von Kunstwerken und historischen Bauten erarbeitet. Dumbarton Oaks erhob hierbei die Forderung nach einer internationalen Kulturorganisation, die in den Rahmen der großen politischen Vereinigungen integriert werden sollte (ebd.; vgl. Hüfner 1996).

Sowohl die amerikanische Initiative als auch die Beratungen der alliierten Bildungsminister in London (1942-1944) ließen den Gedanken an eine universale Organisation für internationale Zusammenarbeit auf geistig-kulturellem Gebiet reifen (ebd.) Vom 01. - 11. 11. 1945 wurde eine vorbereitende Konferenz mit der Ausarbeitung von Richtlinien für eine Kulturorganisation der UN abgehalten. Ein wesentliches Er- 
gebnis dieser Konferenz war die Ausarbeitung und Annahme der Satzung, wofür ein Entwurf der alliierten Bildungsminister und der französischen Regierung vorlag. Des weiteren wurde der Sitz der Organisation bestimmt und ein Abkommen über die Bildung eines vorbereitenden Ausschusses unterzeichnet (Menzel 1957:2f).

Dieses Gremium setzte sich aus je einem Vertreter der Unterzeichnerstaaten der Schlussakte der vorbereitenden Konferenz zusammen, die einen Exekutivausschuss von 15 Mitgliedern wählten. Diesem vorläufigen Organ oblag die Vorbereitung der ersten Hauptversammlung, die Klärung der Verhältnisse der UNESCO zu den UN und anderen Organisationen, die Ausarbeitung von Vorschlägen über die Bereitstellung von Mitteln, sowie die Einsetzung eines Untersuchungsausschusses für Sofortmaßnahmen zugunsten kriegszerstörter Gebiete (ebd.).

Am 16. November 1945 unterzeichneten die Vertreter von 37 Staaten in London die Schlussakte und Verfassung der UNESCO, in deren Präambel die Vertragsstaaten erklären: „Da Kriege im Geist der Menschen entstehen, muss auch der Frieden im Geist der Menschen verankert werden“ (UNESCO 2001a:1; vgl. Hüfner 1996:11f; Menzel 1957:2-3).

Die Arbeit des Ausschusses endete mit dem Beginn der ersten Vollversammlung der UNESCO in Paris (19.11. - 12.12.1946), die weitere organisatorische Fragen erledigte und damit die praktische Arbeit beginnen ließ (Menzel 1957:3).

\subsection{Ziele}

Die UNESCO als Sonderorganisation im System der UN definiert ihr Ziel in Artikel I Absatz 1 ihrer Verfassung ${ }^{5}$ wie folgt:

„Ziel der UNESCO ist es, durch Förderung der Zusammenarbeit zwischen den Völkern in Bildung, Wissenschaft und Kultur zur Wahrung des Friedens und der Sicherheit beizutragen, um in der ganzen Welt die Achtung vor Recht und Gerechtigkeit, vor den Menschen-

\footnotetext{
${ }^{5}$ Nach Artikel XV Abs. 4 sind nur die englische und französische Textfassung verbindlich. Die überarbeitete deutsche Fassung orientiert sich weitgehend an dem im Bundesgesetzblatt veröffentlichten Text (BGBL 1971 II, S. 471-487;1978 II, S. 987-989; 1979 II, S. 419; 1983 II, S. 475) sowie an österreichischen und schweizerischen Textvarianten (Österreich: BGBL, 1949, Nr. 49, vom 9. Juli 1948, S. 252-270; Schweiz: Systematische Sammlung des Bundesrechts der Schweizerischen Eidgenossenschaft; http://www.admin.ch/ch/d/sr/c0_401.html). Insgesamt bemüht sich die deutsche Fassung um Annäherung an einen zeitgemäßen deutschen Sprachgebrauch (UNESCO 2001a:1).
} 
rechten und Grundfreiheiten zu stärken, die den Völkern der Welt ohne Unterschied der Rasse, des Geschlechts, der Sprache oder Religion durch die Charta der Vereinten Nationen bestätigt worden sind“ (UNESCO 2001a:1).

Die Verfassung trat am 4. November 1946 in Kraft, nachdem sie von 20 Staaten ratifiziert wurde. Am 14. Dezember 1946 wurde gemäß Artikel 57 und 63 der UNCharta (UN 1945) die UNESCO mit dem Status einer Sonderorganisation der UN von der Generalversammlung der UN bestätigt. Implizit vertritt die UNESCO eine Politik der Entwicklung im humanistischen Sinne, z.B. durch Förderung der Überwindung des Analphabetentums und der Grundbildung für alle beim Schutz und der Erhaltung des kulturellen und natürlichen Welterbes und beim Beschreiten neuer Wege in der internationalen wissenschaftlichen Zusammenarbeit (Hüfner\&Reuther 1996:5; vgl. UNESCO 2001a).

Um diese Ziele zu verwirklichen, wird die UNESCO laut Artikel I Absatz 2 ihrer Verfassung:

„(a) in allen Massenmedien bei der Förderung der Verständigung und der gegenseitigen Kenntnis der Völker mitwirken und internationale Vereinbarungen empfehlen, die den freien Austausch von Ideen durch Wort und Bild erleichtern;

(b) der Volksbildung und der Verbreitung von Kultur neuen Auftrieb geben

- durch Mitarbeit am Aufbau des Bildungswesens derjenigen Mitgliedstaaten, die dies wünschen;

- durch Institutionalisierung internationaler Zusammenarbeit bei der Förderung des Ideals gleicher Bildungsmöglichkeiten für alle ohne Ansehen der Rasse, des Geschlechts oder wirtschaftlicher oder sozialer Unterschiede;

- durch Empfehlung von geeigneten Bildungsmethoden für die Vorbereitung der Jugend der ganzen Welt auf die Verantwortlichkeiten freier Menschen.

(c) Wissen bewahren, erweitern und verbreiten

- durch Erhaltung und Schutz des Welterbes an Büchern, Kunstwerken und Denkmälern der Geschichte und Wissenschaft sowie durch Empfehlung der dazu erforderlichen internationalen Vereinbarungen an die jeweils betroffenen Staaten;

- durch Förderung der internationalen Zusammenarbeit in allen Bereichen des geistigen Lebens. Dazu gehört der internationale Austausch von Bildungsexperten / Bildungsexpertinnen, Wissenschaftlern / Wissenschaftlerinnen und Kulturschaffenden sowie von Veröf- 
fentlichungen, künstlerischen und wissenschaftlichen Objekten und sonstigem Informationsmaterial;

- durch Einführung geeigneter Formen internationaler Zusammenarbeit mit dem Ziel, alle Veröffentlichungen weltweit frei zugänglich zu machen“ (UNESCO 2001a).

Wie aus dem obigen Zitat in Abschnitt $\mathrm{c}$ bereits deutlich wird, wurde die UNESCO bereits zu ihrer Gründung mit einem Mandat für den Schutz von „Kulturgütern“6 im Sinne eines Welterbes ${ }^{7}$ ausgestattet.

\subsection{Mitgliedschaft und organisatorischer Aufbau}

Alle Mitgliedstaaten der UN haben auch ein Recht auf die Mitgliedschaft in der UNESCO (Art. II der UNESCO-Satzung). Die meisten Angehörigen der UN haben hiervon Gebrauch gemacht. Beide Organisationen haben 191 Mitgliedsstaaten, die UNESCO hat zudem noch sechs weitere assoziierte Mitglieder, z.B. Aruba, Tokelau und die Niederländischen Antillen (vgl. UNESCO 2006; vgl. UN 2006).

Auch Nichtmitgliedstaaten der UN können der UNESCO beitreten. Dies setzt aber nach dem Abkommen von 1946 die Zustimmung des Wirtschafts- und Sozialrates der UN sowie die Aufnahmeempfehlung durch den Verwaltungsrat der UNESCO und die Billigung durch eine Zweidrittelmehrheit der Generalkonferenz selbiger Organisation voraus (Menzel 1957:4).

Als Sonderorganisation der UN ist die UNESCO eine selbständige, mit eigenem Entscheidungsrecht und eigener Finanzhoheit ausgestattete Organisation, die jedoch auf Grund der besonderen Vereinbarungen (Art. 63 der UN-Charta und Art. X der UNESCO-Satzung) in engeren Beziehungen zu den Vereinten Nationen steht und auch an den allgemeinen Einrichtungen der UN teilnimmt (Hüfner 1996:15f).

\footnotetext{
${ }^{6}$ Auf internationaler Ebene, wie auch auf nationaler Ebene wird der englische Rechtbegriff des „Cultural Property" im deutschen Rechtskanon übersetzt mit „Kulturgut“ (vgl. Frigo 2004, Blake 2000, Prott\&O’Keefe 1992).

${ }^{7}$ Der Welterbebegriff wurde in der Verfassung jedoch nicht weiter erörtert, sondern eher als gegebener Begriff verwendet (vgl. UNESCO 2001a).
} 
Die essentiellen Abteilungen der UNESCO sind die Generalkonferenz, der Exekutivrat und das Sekretariat, an dessen Spitze ein Generaldirektor steht. Alle Organe sind in der Satzung festgeschrieben (Art. III). In Artikel IV, V und VI werden die Aufgaben, Zusammensetzung und Verwaltungsverfahren der einzelnen Organe aufgeführt (UNESCO 2001a:1ff).

Die Generalkonferenz ist das oberste Entscheidungs- und Kontrollorgan der UNECO. Sie ist sozusagen der "Souverän“ dieser multilateralen Organisation und tritt alle zwei Jahre zu einer ordentlichen Tagung zusammen. Sie legt die politischen Zielsetzungen und Arbeitsrichtlinien der UNESCO fest, wobei ein vom Sekretariat in Abstimmung mit den Mitgliedsstaaten vorgelegtes zweijähriges Arbeitsprogramm als Diskussionsgrundlage dient. Ferner beruft die Generalkonferenz internationale Staatenkonferenzen ein, nimmt internationale Empfehlungen oder Übereinkommen an und erörtert die Berichte der Mitgliedsstaaten an die Organisation über die Maßnahmen zur Umsetzung von Empfehlungen und Übereinkommen (Hüfner 1996:15f). Nach Beendigung einer Sitzung der Generalkonferenz hat jeder Mitgliedstaat die während dieser Sitzung angenommenen Empfehlungen und Konventionen seinen zuständigen Stellen binnen einem Jahr vorzulegen (UNESCO 2001a:3; Hüfner 1996:15f). Alle Vertragsstaaten sind in regelmäßigen Abständen verpflichtet, über die Verwirklichung von Konventionen und zu Empfehlungen schriftlich Stellung zu nehmen (Hüfner 1996:15f).

Die Generalkonferenz wählt auch die Mitglieder des Exekutivrates, der aus 58 Mitgliedern zusammengesetzt ist. Der Exekutivrat fungiert als Bindeglied zwischen Generalkonferenz und Sekretariat einerseits für die Vorbereitung der Tagesordnung der Generalkonferenz, die Prüfung des Arbeitsprogramms, des entsprechenden Haushaltsplans und andererseits für die Überwachung des Sekretariats bei der Durchführung des von der Generalkonferenz verabschiedeten Arbeitsprogramms (ebd.17).

Das Sekretariat ist die Geschäftstelle der UNESCO und wird von einem Generaldirektor geleitet, der auf Vorschlag des Exekutivrates von der Generalkonferenz für eine Amtszeit von sechs Jahren gewählt wird. Der Generaldirektor ist mit beträchtlichen Kompetenzen ausgestattet; er nimmt ohne Stimmrecht an allen Sitzungen der Generalkonferenz, des Exekutivrates und aller Ausschüsse teil, erstellt regelmäßig Berichte über die Tätigkeit der Organisation, die er den Mitgliedsstaaten und dem Exekutivrat übermittelt, und entwirft das Arbeitsprogramm mit entsprechenden Haushaltvorschlägen für den Exekutivrat (ebd.). 


\section{Die Bedeutung und Transformation des Kulturbegriffs in der UNESCO}

Die Kampagne zur Rettung der Tempel von Abu Simbel am Nil 1959, die militärischen Aktionen gegen die „Perle von Adria“, Dubrovnik/ Kroatien, während des Krieges am Balkan 1991, die absichtliche Zerstörung der Buddha-Statuen von Bamiyan/ Afghanistan 2001, die Anerkennung Auschwitz als kulturelles Erbe der Menschheit, sowie die Auszeichnung der Zápara People als Masterpiece of the Oral and Intangible Heritage of Humanity haben einen Aspekt gemeinsam: sie sind Gegenstand des Diskurses innerhalb der internationalen Staatengemeinschaft, in dessen Mittelpunkt jene Bemühungen stehen, die auf den internationalen Schutz von „kulturellem Erbe“ und „kulturellem Eigentum“ abzielen (vgl. Strasser 2005:52ff).

Auf dem Gebiet des Schutzes von kulturellem Erbe und kulturellem Eigentum sind international viele Akteure tätig (z.B. die WIPO, ICOM, ICOMOS, der Europarat, die OSZE, die Arabische Liga, diverse NGO's) ${ }^{8}$. Die mitunter international wichtigste Organisation ist die UNESCO (ebd.; s.o.).

Während die meisten der oben genannten Organisationen oftmals nur einzelne Aspekte eines kulturellen Erbes zu schützen versuchen oder regionale Interessen verfolgen (z.B. die WIPO, als Teilorganisation der UN, bemüht sich um den Schutz von intellectual property - sprich geistigem Eigentum), bemüht sich die UNESCO konzeptionell um die Möglichkeit eines weltweit umfassenden Schutzes aller Bereiche des kulturellen Erbes der Menschheit und einzelner Gruppen.

Begreift man kulturelles Eigentum und kulturelles Erbe als Konstrukte, so wird deutlich, dass sich ihr essentieller Bestand aus Kultur, Eigentum und Erbe zusammensetzt. Jede wissenschaftliche Disziplin hat ihre eigenen theoretischen Hintergründe und Erklärungsmuster, um diese Phänomene zu deuten und Theorien zu konstruie-

\footnotetext{
${ }^{8}$ Ich gehe hier nicht weiter auf die einzelnen Organisationen ein, sondern bemühe mich ausschließlich um die Darstellung der UNESCO.
} 
ren. Auch die Ethnologie bemüht sich seit ihrer Begründung ${ }^{9}$ darum einen Beitrag zu leisten, um die Wesenheit jener Phänomene zu durchdringen, zu abstrahieren und zu erklären.

In den folgenden Unterkapiteln behandele ich einleitend generelle Aussagen zu einem ethnologisch-kulturwissenschaftlichen Kulturbegriff im Rahmen der UNESCOKonzeptionen, um in den darauf folgenden Kapiteln die UNESCO-Konzeptionen eines kulturellen Eigentums und Erbes darzustellen und zu analysieren.

Zunächst erörtere ich, wie sich der Kulturbegriff der UNESCO veränderte. Dabei greife ich auf theoretische Grundlagen der Ethnologie zurück, um das Wesen des Sachverhaltes aus ethnologischer Perspektive darzulegen bzw. zu skizzieren. Mein Anspruch kann dabei nicht sein, dass ich eine umfassende Darstellung jedes einzelnen Aspektes verwirkliche, sondern vielmehr den jeweiligen Kern des Phänomens im Kontext dieser Arbeit fasse und inn in seiner Bedeutung für die UNESCO Konzeptionen reflektiere. ${ }^{10}$ Bei der Ausarbeitung jedes einzelnen Aspektes erachte ich es als unabdingbare Voraussetzung, zu Anfang jeweils die basissemantischen Bedeutungen bzw. die Begriffsgeschichte der einzelnen Begriffe darzustellen, um darauf aufbauend wissenschaftliche Abstraktionen zu formulieren.

\subsection{Statischer Kulturbegriff versus Dynamischer Kulturbegriff}

Bei der grundlegenden Betrachtung der UNESCO-Konzeptionen von Eigentum und Erbe wird deutlich, dass sich beide Konzeptionsformen durch eine besondere Qualität auszeichnen. Diese Qualität ist die des „kulturellen“. Sie hebt die eigentlichen Begrifflichkeiten des Erbes und des Eigentums auf eine andere Abstraktions-, Deutungs- und Verstehensstufe und kann als grundlegender Bestandteil bzw. als fundamental interpretiert werden.

Ich werde daher einleitend einige Aspekte des Kulturbegriffs und seiner Abstraktionen darstellen, die, wie ich meine, für ein weiteres und vertiefendes Verständnis der Thematik dieser Arbeit unerlässlich sind. Der Wandel des Kulturbegriffs der

\footnotetext{
${ }^{9}$ Auf die fachspezifische Diskussion über die Anfänge ethnologischer Forschung werde ich nicht weiter eingehen. Es sei aber erwähnt, dass die Ethnologie als Universitätsfach in der zweiten Hälfte des 19. Jhr. etabliert wurde (Haller 2005:23).

${ }^{10}$ Diese Voraussetzung gilt für alle darzustellenden Facetten im Zusammenhang mit den Eigentums- und Erbekonzeptionen der UNESCO.
} 
UNESCO ist, wie ich noch aufzeige, in einem historischen Zusammenhang mit dem konzeptionellen Wandel von kulturellem Eigentum zu kulturellem Erbe zu sehen. Da durch die Emanzipation der ehemaligen Kolonien neue kulturpolitische Forderungen und Ziele entstanden, denen ein statisches Verständnis von Kultur nicht mehr gerecht werden konnte (s. Kapitel 5\&6). Des weiteren möchte ich gleich zu Beginn meiner Erläuterungen festhalten, dass ich bei der Betrachtung von Kultur prinzipiell den Plural voraussetze. Vorteilhafter wäre es, wenn ich eher von kulturellen Kontexten schriebe, um dem des Nomens immanenten Verständnis von Kohärenz, Gleichförmigkeit und Abgeschlossenheit zu entgehen. Der besseren Opernationalisierbarkeit wegen verwende ich aber vorzugsweise den Singular. Des weiteren erörtere auch nur jene Aspekte eines Kulturbegriffs, die im direkten Zusammenhang mit den UNESCO-Konzeptionen von Kultur stehen.

Das Phänomen Kultur besitzt für die Ethnologie eine analoge Valenz (explanatory importance) wie der Krankheitsbegriff für die Medizin oder die Gravitation für die Physik (Kroeber\&Kluckhorn 1952:3). Ob es sich dabei um einen Gegenstand der Forschung, um einen Erklärungsansatz oder beides handelt, wird von vielen Autoren unterschiedlich beantwortet (Beer 2003:60). Im Falle der UNESCO handelt es sich, wie wir noch lesen werden, um eine Abstraktion, die sich mit den Zeiten wandelt und den neuen Herausforderungen aus Sicht der UNESCO anpasst (vgl. Schöfthaler\&Dyroff 1996:7ff).

\subsection{Begriffsgeschichte}

Die theoretische Konstruktion von "Kultur" setzte im 18. Jahrhundert ein (Nünning 2003:19; Müller 2003:14), während der Begriff „Kultur“ im Lateinischen bereits ab dem 2. Jahrhundert v. Chr. Verwendung findet. Er tritt zunächst im Zusammenhang mit dem Bodenbau auf. Das lateinische Verbum colere wird von dem Hauptwort cultura abgeleitet und bedeutet „bebauen“, „,bearbeiten“, „veredeln“ und „pflegen“. Grundlegend war die Idee des bewussten Hegens und Pflegens des Ackers bzw. Gartens, z.B. in Marcus Terentius Varro (116-27 v. Chr.) Schrift über die Landwirtschaft (Res rusticae; Müller 2003:15; vgl. Nünning 2003:19f). Man verstand diese Idee - laut Müller - als die Fortsetzung der Schöpfung (2003:14). 
Die römische Antike bezeichnete mit cultura und cultus nicht nur die naturbezogenen Tätigkeiten des Menschen und deren landwirtschaftliche Ergebnisse (cultura agri), sondern auch die religiöse „Pflege“ des Übernatürlichen (cultus deorum) und entsprechend der griechischen paideia (gr. „Erziehung, „Unterricht“, „Züchtigung“, Wissenschaft“, „Bildung“) als die wissenschaftliche und künstlerische „Pflege“ der individuellen und sozialen Voraussetzungen des menschlichen Lebens (Nünning 2003:19; vgl. Müller 2003:13f).

Marcus Tullius Cicero (106-43 v. Chr.) verwandte in seinen Tuskanischen Gesprächen (Tuscanae Disputationes II) den Begriff cultura erstmals in der übertragenen Bedeutung „cultura autem animi philosophia est“ (Cicero 1957 [45v.Chr.]: 141; vgl. Nünning 2003:19; vgl. Müller 2003:15f). Müller übersetzt dies als „Veredelung und Pflege der Natur im Menschen, als Bändigung der Leidenschaft und Bildung des Geistes, wie sie die Beschäftigung mit der Philosophie vermittle“ (Müller 2003:15f; vgl. Nünning 2003:19; vgl. Hansen 1995:13). Beer betont, dass Cicero damit einen der Grundsteine für unser heutiges Verständnis von Kultur legte (2003:61).

In diesem Sinne behauptete und entwickelte sich der Begriff im gelehrten Schrifttum von der Spätantike über das Mittelalter und die Renaissance bis hin in die Neuzeit (Steinbacher 1976:14; vgl. Müller 2003:16) als abstrakter und selbständiger Begriff (Nünning 2003:19). Das erweiterte Bedeutungsfeld wird durch den Gegenbegriff einer „Natur"11 begrenzt, die es zu bearbeiten und zu domestizieren gilt (ebd.).

Kant versteht den Begriff in seiner Schrift „Anthropologie in pragmatischer Hinsicht“ als vernunftgeleitete Gegenmaxime zur „Rohigkeit“ der menschlichen „Natur“ bzw. als „pragmatische Anlage der Zivilisation durch Kultur“ (Kant 1968:676). Der Mensch sei „durch seine Vernunft bestimmt [...] sich durch Kunst und Wissenschaft zu kultivieren, zu zivilisieren und zu moralisieren“12 (ebd.; vgl. Müller 2003:16; vgl. Kroeber\&Kluckhorn 1952:16,42f). Meist benutzte man zu dieser Zeit und im 19. Jahrhundert den Begriff der Kultur synonym mit Zivilisation, dem damals in England und Frankreich üblicheren Wort (Beer 2003:61).

Der Begriff der „Kultur“ im ethnologisch-kulturwissenschaftlichen Sinne bildet sich in der zweiten Hälfte des 18. Jahrhunderts als erstes in Deutschland heraus. Eine klar

\footnotetext{
${ }^{11}$ Zum Verhältnis und eingehenderen Verständnis der Begriffe Natur und Kultur s. Hauser-Schäublin 2001.

12 Müller (2003:16) unterscheidet „Zivilisation“ und „Kultur“ damit, das ersteres eher als die allgemeine, gesellschaftliche Veredelung der Natur zu verstehen ist und letzteres als die Persönliche. Ich gehe hier nicht weiter auf die einzelnen sprachlichen Bedeutungen, Unterschiede und Besonderheiten von Zivilisation und Kultur ein. Näheres zur Begriffsgeschichte findet sich bei Pflaum 1967, Fisch 1992, Müller 2003 und Nünning 2003.
} 
konzeptualisierte Gestalt gewinnt der Begriff erstmals bei Johann Gottfried von Herder (1744-1803), namentlich in Buch VIII und IX seiner „Ideen zur Philosophie der Geschichte der Menschheit“ (Müller 2003:17; vgl. Kroeber\&Kluckhorn 1952:37f). Nachfolgend entstehen im 19. Jahrhundert, wohl nicht zuletzt in Reaktion auf die Entdeckungsreisen und kolonialen Erfahrungen, eine zunehmende Zahl von „Kulturgeschichten“, unter denen die zehnbändige „Allgemeine Culturgeschichte der Menschheit" von Gustav Klemm (1802-1867) das größte Interesse fand (vgl. Müller 2003:17; Kroeber\&Kluckhorn 1952:13f, 30ff, 285f; vgl. Steinbacher 1976:16f; vgl. Berger 1995:15). Das Werk wurde auch im Ausland zur Kenntnis genommen, u.a. von Edward Burnett Tylor, dem Begründer der britischen Ethnologie. Tylor übernahm von Klemm den Herder'schen Kulturbegriff im Sinne des Ausdrucksganzen der Lebensart eines Volkes ${ }^{13}$ und führte inn in sein Werk „Primitive Culture" von 1871 ins Englische ein (Müller 2003:17; Kroeber\&Kluckhorn 1952: 11, 14; Berger 1995:15). Taylors Definition (s.u.) umfasst die Totalität von Kultur und kann als Ausgangspunkt für alle weiteren Kulturbegriffe und Konzepte angesehen werden (Haller 2005:31).

\subsection{Kultur im ethnologisch-kulturwissenschaftlichen Sinne}

Auf der einen Seite bemerkt der amerikanische Ethnologe Edward Adamson Hoebel in seinem Werk "Man in the primitive world“ (1958:7), dass das Kulturkonzept den bedeutendsten Einzelbeitrag der Ethnologie darstelle, während sich auf der anderen Seite ebenso konstatieren lässt, dass diese Einschätzung - laut Müller - kaum einer in der Ethnologie teilt (2003:23) bzw. man bei Betrachtung der einschlägigen Literatur auf die Erkenntnis stößt, dass es kein überzeugendes Konzept gibt (Müller 2003:23; vgl. Kroeber\&Kluckhorn 1952:70, 357; vgl. Goodenough 1981: Vl; vgl. Kessing 1994:310; vgl. Hansen 1995:5; vgl. Richter 1999:163f). Mit Alan Dundes lässt sich hinzufügen, dass selbst über die Schlüsseltermini keine Einigkeit besteht (1968:157). Einige Autoren, wie z.B. Chaterine Lutz und Lila Abu-Lughod fordern

\footnotetext{
${ }^{13}$ Der Begriff „Volk“ ist laut Elwert (1999:400) ein emotional hoch aufgeladener Ausdruck mit stark schwankendem Inhalt. Mal sei eher „Ethnie“, mal eher „Nation“, dann gar die „breite Masse“, die „einfachen“ Mitglieder einer Gesellschaft, oder die „Träger bäuerlicher Kultur" gemeint. Ein weiterführendes Verständnis von „Volk“ findet sich bei E.W. Müller 1980.
} 
gleich, den Begriff der Kultur aus antiessentialistischen ${ }^{14}$ Gründen überhaupt nicht mehr zu verwenden, da er Kohärenz, Gleichförmigkeit und Zeitlosigkeit unterstelle (Lutz\&Abu-Lughod 1990:79, vgl. Müller 2003:23; vgl. Brumann 1999; s.u.).

Appadurai (1996:12) kritisiert sogar, dass manche Autoren in der Ethnologie von einer „physischen Substanz“ ausgingen. Kultur werde in „biologistischer“ Weise ähnlich dem Rassekonzept ${ }^{15}$ verwendet. Demgegenüber stellt Beer fest: „[d]ass grade das Kulturkonzept in der Abgrenzung zu Vorstellungen von Rasseunterschieden entwickelt wurde, dadurch seine Bedeutung erhielt und zur wissenschaftlichen Überwindung dieser Vorstellungen beigetragen hat“ (2003:69). Sie fordert daher, das ethnologische Kulturverständnis einer breiten Öffentlichkeit zugänglich zu machen, um einem Missbrauch durch nationalistisch ausgerichtete Gruppierungen zu erschweren (Beer 2003:69) und Brumann resümiert: „Any scientific concept is a simplifying construct and has its costs, but once the advantages have been found to outweight these costs it should be employed with a clear conscience“ (1999:13).

Was ist denn nun Kultur im ethnologisch-kulturwissenschaftlichen Sinne? Welche Konzeptionen gibt es und welcher bedient sich die UNESCO?

Ein Problem bei der Beschäftigung mit dem Kulturbegriff besteht darin, dass es einen Alltagsbegriff gibt. Kultur kann verstanden werden im Sinne von Kunst, Musik, Theater, Literatur, Architektur, bildender Kunst, Eigentum, Erbe, Tradition Kulturorganisation, Kulturministerium, Kulturbehörde, Kulturbeutel, als unveränderliches Merkmal von Menschen ${ }^{16}$, im Sinne von Gemeinschaft und auch als wissenschaftliches Konzept. Die ersten wissenschaftlichen Definitionen und Erläuterungen zum Kulturbegriff kamen nach Ansicht Beers (2003:61) aus der Ethnologie und wurden von anderen Wissenschaften übernommen. Folgt man jedoch Müller (2003:16f) und Nünning

\footnotetext{
${ }^{14}$ Für ein einführendes Verständnis postmoderner Theorien und antiessentialistischer „Konstruktionen“ s. Derrida 1972.

${ }^{15}$ Eingehendere Betrachtungen zum Begriff und zur Konstruktion von Rasse und die damit verbundenen Diskussionen können hier leider nicht weiter verfolgt werden.

${ }^{16}$ Grade in den Medien wird diese Auffassung von Kultur oft vermittelt. Kultur wird eher verstanden als eine Anzahl klar unterschiedener, beständiger und relativ statischer Merkmale von Menschen mit gemeinsamer Abstammung. In Diskussionen über multikulturelle Gesellschaften (Stichwort: Leitkultur) oder auch in Stellungnahmen fremdenfeindlicher Gruppierungen, aber auch in den populären Politdiskussionen (Stichwort: Kampf der Kulturen) wird ein solcher Kulturbegriff missbraucht (Beer 2003:60f; zum heute in der Ethnologie und den Kulturwissenschaften gängigen Verständnis von Kultur komme ich später).
} 
(2003:21f) gilt es dem Verdienste Herders ${ }^{17}$, das Phänomen der Kultur als erster ausführlicher zu fassen.

Wie oben bereits beschrieben ist die wissenschaftliche Diskussion über das Phänomen Kultur groß. Ein Kulturkonzept ist ein Entwurf bzw. eine Definition von Kultur und dem daraus resultierenden Verständnis, wie Kultur in einem speziellen Zusammenhang (z.B. psychologisch, evolutionistisch, soziologisch) oder in einer bestimmten Epoche (z.B. Aufklärung, Humanismus, Moderne, Post-Moderne) zu verstehen ist oder eben verstanden wurde (vgl. Kroeber\&Kluckhorn 1952). Kroeber und Kluckhorn haben in inrem Werk "Culture: a Critical Review of Concepts and Definitions" von 1952 über 150 verschiedene Definitionen zusammengetragen und unter diversen Gesichtpunkten kategorisiert. Sie wiesen nach, dass Tylor 1871 zwar als erster den Begriff Kultur umfassender definierte, sich dabei aber wie oben dargestellt auf Klemm bezog, der wiederum in der Tradition Herders stand (vgl. Nünning 2003; vgl. Müller 2003). Die Autoren typisierten die verschiedenen Definitionen und teilten sie in beschreibende, historische, normative, psychologische, strukturale, genetische und unvollständige Definitionen (Kroeber\&Kluckhorn 1952:77ff, vgl. Beer 2003:62f).

Kessing wiederum teilte die Schwerpunkte in der Auseinandersetzung um den Kulturbegriff und die unterschiedlichen Kulturkonzepte der 50'er bis 70'er Jahren in adaptive und ideationale (ideational) oder, wie Beer (2003:63) es nennt, mentalistische Kulturtheorien ein (vgl. Keesing 1974:74ff).

Seit der Kulturdefinition Tylors veränderte sich die Betonung der verschiedenen definierenden Merkmale. Das Kulturkonzept der UNESCO vollzog in eben solcher Weise einen Wandel im Laufe der Jahrzehnte seit dem Bestehen der Organisation (s.u.).

Beers Ausführungen zufolge steht hinter den meisten Definitionen jedoch ein Kern an gemeinsamen Grundannahmen (Beer 2003:66). Der kleinste gemeinsame Nenner des Großteils ethnologisch-kulturwissenschaftlicher Definitionen bestehe darin, dass kulturelles Verhalten erlernt, Kultur überindividuell und historisch gewachsen sei, aus einer Anzahl von empirisch fassbaren Merkmalen, Kenntnissen, emotionalen Verhaltensroutinen oder Gewohnheiten besteht; eine Gesamtheit von Merkmalen darstelle, deren Ränder sich unscharf und dynamisch überschneiden und das Kultur nie als völlig homogen zu betrachten wäre. Kultur ist eine Abstraktion, die zudem keine

\footnotetext{
${ }^{17}$ Johann Gottfried Herder (1744-1803) war deutscher Philosoph, Theologe und Dichter, dessen Schriften wesentlich die deutsche Klassik und Romantik beeinflusst und die deutsche Sprach- und Geschichtswissenschaft mit begründet haben (Keßler 2005:1ff).
} 
ewige und wahre Bedeutung hat (vgl. Kroeber\&Kluckhorn 1952). Stattdessen ist Kultur das, was die Wissenschaft zu ihrer Zeit und innerhalb ihrer Disziplin oder auch theoretischen Ausrichtung darunter versteht (Beer 2003:66f).

Aus der mutmaßlich paradoxen Feststellung, dass Kultur eine uneinheitliche Einheit ist, die meist nur zu einem bestimmten Zeitpunkt empirisch erfassbar wird, sich aber ständig in Veränderung befindet, resultiert die von Schiffauer gefolgerte Forschungsstrategie: Kultur „[...] muss einmal betrachtet werden, als ob sie ein vergleichsweise geschlossenes System von Standards und Regeln darstellte, und zum anderen als ob sie ständig im Fluss wäre“ (Schiffauer 1997:149, Hervorhebungen im Original; Beer 2003:68).

\subsection{Das Kulturkonzept der UNESCO: von den Anfängen bis heute}

Der inhaltliche bzw. thematische Rahmen des kulturellen Erbes und des kulturellen Eigentums hängt zweifelsohne vom zugrunde gelegten Kulturkonzept ab (Schneider 2005:4). Im vorangegangenen Teil wurde deutlich, dass es keineswegs eine einheitliche und einfache Lösung gibt, um sich dem Phänomen Kultur zu nähern. Ich habe bisweilen auch noch keine Definition von Kultur vorgestellt, da ich mich ausschließlich auf diejenigen beschränken werde, die bei der UNESCO Verwendung finden. Eriksen bemerkt hierzu: „it cannot be maintained that all, or even nearly all, the writings published under the aegis of UNESCO share a common perspective on culture [...]' (2001:128).

Da der thematische Rahmen der einzelnen UNESCO Konzepte, wie oben bereits angedeutet, vom jeweiligen Kulturkonzept abhängig ist, wird sich zeigen, dass die UNESCO zweigleisig fährt. Auf der einen Seite haben wir es in sehr verschiedenen Zusammenhängen nach wie vor mit engen, ästhetisch determinierten, adaptiven Kulturkonzepten zu tun; auf der anderen Seite werden wir die Anwendung eines weiten, mittlerweile von der UNESCO favorisierten, ethnologisch-anthropologischen Kulturbegriffs erkennen (vgl. Schneider 2005a:4f, ebd. 2005b:40). Meiner Ansicht nach lässt sich mutmaßlich feststellen, dass diese Zweigleisigkeit in direktem Zusammenhang mit der Genese der UNESCO Konzeptionen zu sehen ist (s.u.).

Als die UNESCO im November gegründet wurde, lag die Niederschlagung des Faschismus noch nicht weit zurück. Die Gründer der UNESCO erwarteten, dass die 
kulturelle Zusammenarbeit der Völker diesen die Mittel in die Hand gäbe, sich Angriffen auf die Würde des Menschen zu widersetzen. In die Gründungsidee der UNESCO ging daher die Idee eines humanistischen Verständnisses von Kultur als eine den Geist und die Freiheit der Menschen prägende Kraft ein (Albert 2002:32; Schöfthaler\&Dyroff 1996:7ff).

Herders teleologisches Verständnis von Kultur als historisch gewordene, individuelle Lebensweise von Völkern und Nationen spiegelt eben diese Bedeutung von Kultur als einen der zentralen Begriffe der Fortschrittskonzeption der europäischen Aufklärung und des Humanismus wider (Nünning 2003:20). Tylors Definition, als rein summarische bzw. adaptive und bis heute vielzitierte Definition, stand exakt in dieser Tradition der Aufklärung und den Anfängen der UNESCO (Müller 2003:17; vgl. Nünning 2003:20; vgl. Schöfthaler\&Dyroff 1996:7ff; s.o.). Bis heute ist diese Definition eine der bekanntesten (Beer 2003:61). Tylors Definition von Kultur lautet:

„Cultur oder Civilisation [sic.] im weitesten ethnographischen Sinne ist jener Inbegriff von Wissen, Glauben, Kunst, Moral, Gesetz, Sitte und allen übrigen Fähigkeiten und Gewohnheiten, welche der Mensch als Glied der Gesellschaft sich angeeignet hat. Der Zustand der Cultur in den mannichfaltigen [sic.] Gesellschaftsformen der Menschheit ist, soweit er sich auf Grundlage allgemeiner Principien [sic.] erforschen läßt, ein Gegenstand, welcher für das Studium der Gesetze menschlichen Denkens und Handelns wohl geeignet ist " (Tylor 1873b:1).

Zu kritisieren ist an dieser Definition, nach Ansicht Beers, dass sie nicht das Wesentliche abstrahiert. Eine solche Aufzählung bzw. additive Definition als Summe sämtlicher Objektivationen der Kultur eines Ethnos (Müller 2003:18; Kroeber\&Kluckhorn 1952:5f) kann nie vollständig sein und hat deshalb auch nur beschränkten Nutzen (Beer 2003:62). Der letzte Teil von Tylors Definition führt jedoch über eine Aufzählung hinausgehende Merkmale an: „Kultur umfasst eben auch Fähigkeiten und Gewohnheiten, die der Mensch als Mitglied einer Gesellschaft erworben habe" (ebd.).

Selbst Lévi-Strauss, der die weitere Entwicklung der UNESCO Konzeption von Kultur wesentlich seit den 50'er Jahren beeinflusste, bekannte sich vor wenigen Jahren noch expressis verbi zu dieser Definition und rezitierte die von inm hochgepriesene Tylor'sche Formel zur Bestätigung einem Interview-Partner aus dem Kopf (LéviStrauss\&Eribon 1989:240; vgl. Müller 2003:23), obwohl er selbst eher der Symbolischen Ethnologie und vor allem dem Strukturalismus zuzuordnen ist (Müller 2003:26) und Kulturen als ethnisch integrierte Symbol- oder Sinnsysteme mit je eigener Textur 
versteht, deren bedeutungsträchtige "Codes" Orientierungs- und Regelvorgaben für das Verhalten und Handeln bereitstellen (Müller 2003:25; vgl. Reckwitz 2000:31, 85). Eine heute in der Ethnologie gängige Darstellung des Kulturkonzepts der Symbolischen Ethnologie stammt von Clifford Geertz. Kultur sei zu verstehen als: „webs of significance man himself has spun“ (Geertz 1975: 5, 10; vgl. Müller 2003:26; vgl. Nünning 2003: 33ff). „Kulturen“ können in diesem Sinne als hochkomplexe Kommunikationssysteme interpretiert werden. Lévi-Strauss, wie auch Geertz, begriff Symbole als "Zeichen“, ein Ensemble von „Zeichen“ als "Codes“ einer innergesellschaftlichen Verständigung (vgl. Lévi-Strauss 1949; 1981; Geertz 1969:3). Alle menschlichen Beziehungen beruhen auf dem Austausch von Nachrichten (Levi-Strauss 1969:178; vgl. Müller 2003; vgl. Nünning 2003).

Im Laufe der 50'er und 60'er Jahre änderte sich das Kulturkonzept der UNESCO auf dem Hindergrund der einsetzenden Entkolonialisierungsprozesse in Afrika und Asien (Albert 2002:32). Mit dem Aufbrechen der kolonialen Systeme und dem erwachenden Selbstbewusstsein der jungen Nationen in den VN kamen erste Initiativen innerhalb der Völkergemeinschaft auf, sich mit der kulturellen Identität der Völker näher zu befassen (van Hasselt 1996:61). Wegweisend für das weitere Kulturverständnis war der Text „Race and History" von Lévi-Strauss, den er 1951 im Auftrag der UNESCO verfasste. In diesem Text lassen sich zwei Prämissen festmachen. Die erste Prämisse ist die eindeutige Ablehnung des Rassismus als Festlegung und Übertragung genetischer Merkmale von Menschen auf deren Lebensausdrücke und Lebensstile (Albert 2002:33).

Die zweite Prämisse ist die Bewertung aller Kulturen als gleichwertig in ihrer Würde und in ihrem Stellenwert innerhalb des weltweiten kulturellen Gefüges. Für das Verständnis kultureller Entwicklungsprozesse sind nicht Stadien oder Etappen innerhalb einer linearen Entwicklung anzulegen, die vom gleichen Ausgangspunkt herkommen und zum gleichen Ziel führen müssen (Albert 2002:32; vgl. Levi-Strauss 1975), sondern die Vielschichtigkeit der Traditionen und Lebensweisen. Der linearen Hierarchisierung von Kulturen nach einem euroamerikanischen „Vorbild“ der Entwicklung war somit eine eindeutige Absage erteilt (Albert 2002:32).

Durch Lévi-Strauss diffundiert der in der Kulturanthropologie diskutierte Kulturrelativismus in das Kulturverständnis der UNESCO. Gleichzeitig wurde damit die evolutionistische Vorstellung vom linearen Fortschreiten der kulturellen Entwicklung einer heftigen Kritik unterzogen. Die Basis war geschaffen, die Kulturen der Welt nicht nur 
in ihrer Vielfalt zu akzeptieren, sondern auch die notwendige Toleranz einzufordern, jede einzelne unabhängig vom Modus ihrer Entwicklung als gleichwertig zu betrachten (Albert 2002:33).

Mit diesen wesentlichen Positionen zum Kulturverständnis beschloss die Generalversammlung der UNESCO am 16. November 1972 die Welterbekonvention und vollzog gleichsam den Wandel vom konzeptionellen Schwerpunkt eines kulturellen Eigentums hin zur Hervorhebung eines kulturellen Erbes (Albert 2002:33) ${ }^{18}$.

1982 rief die UNESCO in Mexiko-Stadt zur Weltkulturkonferenz auf. Inhaltlich behandelte die Konferenz die neue Kulturpolitik der Organisation. Es ging um kulturelle Identität, Kultur und Gesellschaft, das Erbe, die Bedingungen für die Freiheit der Kunst und die internationale kulturelle Zusammenarbeit. Kultur wurde dabei als Hauptelement des Entwicklungsprozesses definiert, das dazu beiträgt, die Unabhängigkeit, Souveränität und Identität der Nationen zu stärken. Ziel einer wahrhaftigen Entwicklung sei das dauerhafte Wohlergehen und die Entfaltung eines jeden Menschen. Endziel jeder Entwicklung müsse der Mensch selbst in seiner menschlichen Würde sein (van Hasselt 1996:63).

Die Weltkulturkonferenz in Mexiko schuf einen seither in der UNESCO und damit in der internationalen Gemeinschaft akzeptierten weiten ethnologischkulturwissenschaftlichen Kulturbegriff, der sich endgültig von der "Ornamentkultur“ und der alten Tylor'schen Formel distanzierte (van Hasselt 1996:63; vgl. UNESCO 1982:1).

"Therefore, expressing trust in the ultimate convergence of the cultural and spiritual goals of mankind, the Conference agrees: that in its widest sense, culture may now be said to be the whole complex of distinctive spiritual, material, intellectual and emotional features that characterize a society or social group. It includes not only the arts and letters, but also modes of life, the fundamental rights of the human being, value systems, traditions and beliefs" (UNESCO 1982: Präambel).

Der Text macht jedoch nicht deutlich, dass innerhalb der Kulturen selbst dynamische Prozesse stattfinden, dass es zu Brüchen und Konflikten von Moderne und Tradition, von Eliten und Benachteiligten innerhalb ihres kulturellen Kontextes kommt, so dass

\footnotetext{
${ }^{18}$ Diese Entwicklung erforderte in ihrer Logik eben auch die Weiterentwicklung eines geeigneten Kulturkonzepts. In den folgenden Kapitel zu kulturellem Eigentum und kulturellem Erbe werde ich eingehender auf die Gründe und Begebenheiten des konzeptionellen Wandels eingehen.
} 
Kultur nicht immer das einigende und prägende Element ist, wie es nach der obigen Definition scheint (van Hasselt 1996:63).

Einen der prägnantesten Höhepunkt der UNESCO-Initiativen zum kontextuellen Verständnis von Kultur stellt die 1991 beschlossene Einsetzung einer unabhängigen Weltkommission für Kultur und Entwicklung unter dem Vorsitz des ehemaligen Generalsekretärs der VN, Javier Pérez de Cuéllar dar. Der Kommission gehörten unter anderem zwölf bedeutende Persönlichkeiten aus allen Weltregionen und unterschiedlichen Disziplinen an. Ehrenmitglieder waren z.B. der Literaturnobelpreisträger Derek Walcott, die Friedensnobelpreisträgerin Aung San Sun Kyi und der Ethnologe Claude Lévi-Strauss (van Hasselt 1996:65).

1994 verfassten Pérez de Cuéllar und weitere Autoren die Abschlussdokumentation „Our Creative Diversity“. Im Mittelpunkt steht die Aussage, dass jegliche Entwicklung, die von ihrem menschlichen oder kulturellen Kontext abgetrennt wird, „Wachstum ohne Seele" (Schöfthaler\&Dyroff 1997:21) sei. Es reiche nicht mehr aus, kulturelle Faktoren in der Entwicklungsplanung lediglich nebenbei zu berücksichtigen und es sei ganz und gar inakzeptabel, sie zu einem „helfenden“ oder „hindernden“ Parameter für Wirtschaft und Gesellschaft herabzustufen (van Hasselt 1996:65; vgl. Pérez de Cuéllar 1998:7ff; vgl. Schöfthaler\&Dyroff 1996:12ff). Die Autoren versuchten der Instrumentalisierung von Kultur als einem Mechanismus zur Erreichung ökonomischen Wachstums nach euro-amerikanischem Vorbild einen Ansatz entgegenzustellen, der Kultur als ein aus sich selbst heraus legitimiertes Orientierungssystem fasst. Kultur wird einerseits als funktional für die wirtschaftliche Entwicklung gesehen, und andererseits als die soziale Grundlage eben dieser Entwicklung. Entwicklung und Wirtschaft sind gleichermaßen Teil der Kultur eines Volkes (Schöfthaler \&Dyroff1997:21ff; vgl. Albert 2002:33f; vgl. Pérez de Cuéllar 1998:7ff).

Die derzeitig von der UNESCO favorisierte Definition von Kultur stimmt mit den Beschlüssen der Weltkonferenz über Kulturpolitik (Mexiko City, 1982), der Weltkommission über Kultur und Entwicklung (Unsere kreative Vielfalt, 1995) und der Zwischenstaatlichen Konferenz über Kulturpolitik zur Entwicklung (Stockholm 1998) überein (UNESCO 2002c:4). Explizit zum Ausdruck kommt die Definition in der Allgemeinen Erklärung zur kulturellen Vielfalt (Universal Declaration on Cultural Diversity; UNESCO 2001b:Präambel). In Folge entstand die Konvention zum Schutz der Vielfalt kultureller Inhalte und künstlerischer Ausdrucksformen (Convention on the Protection and Promotion of the Diversity of Cultural Expressions; UNESCO 2005f) auf Grund- 
lage eben dieser ethnologisch-kulturwissenschaftlichen Konzeption von Kultur (Reuther 2005:135ff).

Die UNESCO Definitionen von Kultur entwickelten sich von einem anfänglich ästhetischen und adaptiven Konzept im Sinne Tylors auf Grund eines policy-turns der UNESCO (s.u.) und der Emanzipation der ehemalig kolonialisierten Staaten hin zu einem offenen ethnologisch kulturwissenschaftlichen Konzept, um neueren kulturpolitischen Bestrebungen der UNESCO z.B. in der so genannten „Magna Charta für Kulturpolitik“19 (Metze-Mangold 2005:1) gerecht zuwerden.

Beide Konzeptionen bestehen gegenwärtig implizit in den verschiedenen Konzeptionen fort. Während in den älteren Konzeptionen zu kulturellem Eigentum ein adaptives bzw. additives Kulturkonzept als qualitatives Fundament der Eigentumskonzeptionen besteht, wird in den neueren Erbekonzeptionen seit der Welterbekonvention ein offen-dynamisches Konzept bevorzugt, das sich seit der Mexiko Konferenz von 1982 entwickelte.

\subsection{Das Unbehagen mit dem Kulturbegriff der UNESCO}

Die Verwendung des Kulturbegriffs bei der UNESCO lässt, wie oben bereits angemerkt, zwar keine eindeutigen Schlüsse auf ein einheitliches Konzept zu, aber auf ein rezent favorisiertes.

Demgegenüber bemerkt Eriksen, dass der hochgelobte und vielgepriesene UNESCO Report "Our Creative Diversity" von 1996 trotz der Bemühungen der Autoren kein schlüssiges Kulturkonzept aufweist (Eriksen 2001: 128f; 130). Der Autor führt an, dass der Bericht einerseits die Beziehung zwischen Kultur als künstlerische Betätigung und Kultur als "way of life" darstellt und in diesem Sinne zu Beginn des Reports Marshall Sahlins mit der sinngemäße Aussage zitiert wird, das „every human activity, including those relating to development and the economy, has a cultural component or dimension" (Eriksen 2001:131; vgl. Pérez de Cuéllar 1996:21) und andererseits

\footnotetext{
${ }^{19}$ Im Kern geht es um die Fragen, ob kulturelle Produkte angesichts ihres Doppelcharakters, handelbare Waren und identitätstiftende Werte zu sein, den Gesetzen des Weltmarkts allein unterliegen sollen oder künftig auch Gegenstand staatlicher Kulturpolitik sein dürfen und wie tragfähige Kulturmärkte aufzubauen sein (MetzeMangold 2005:1ff). Weiterführende Informationen finden sich bei Metze-Mangold 2005 und in diesem Zusammenhang in der UNESCO Convention on the Protection and Promotion of the Diversity of Cultural Expressions ebenfalls von $2005(f)$.
} 
Kultur als rein künstlerische Produktion bzw. als Kunstschaffen konzeptionalisiert. Eriksen kommentiert diese Feststellung mit den Worten: "little effort is made to distinguish between the two perspectives"(2001:131).

Eriksen konstatiert demgegenüber noch ein weiteres, seiner Ansicht nach schwerwiegenderes Problem bei der Kulturkonzeption im Pérez de Cuéllar Report. In großen Teilen der Schrift wird Kultur definiert als ,,something that can easily be pluralized, which belongs to a particular group of people, associated with their heritage or ,roots"' (Eriksen 2001:131). Andererseits betonen die Autoren des Reports, dass Globalisierung, Kreolisierung ${ }^{20}$, äußere Einflüsse und Impulse ebenfalls kulturelle Phänomene darstellen. Bei diesen beiden Annahmen muss nach Ansicht Eriksens analog zwischen zwei unterschiedlichen Kulturtheorien der aktuellen Ethnologie/ Anthropologie unterschieden werden. Laut Eriksen bezieht sich die erste Annahme auf den Kulturrelativismus, Struktur-Funktionalismus und den Strukturalismus, während sich die zweite Behauptung eher auf dekonstruktivistische Trends und poststrukturalistische Arbeiten bezieht (ebd. 131f).

In einer nachvollziehbaren Analyse des Reports gelangt Eriksen in seinem Artikel zu der Aussage, dass:

",Throughout the report, cultures are implicitly and explicitly seen as rooted and old, shared within a group, to be treated 'with respect' as one handles aging china or old aunts with due attention to their fragility. [...] Although it is said explicitly that any culture's relationship with the outside world is 'dynamic', UNESCO cultures remain island or at least peninsulas"' (Eriksen 2001:132; vgl. ebd. 1993:1).

Von einer einheitlichen Auffassung dessen, was Kultur sei und was nicht, scheint die UNESCO, wie ich meine, weit entfernt zu sein. Trotz ihrer Bemühungen, kulturelle Vielfalt und ein prozesshaftes und offenes Verständnis von „Kultur“ zu proklamieren. Erich Kasten (2004:21) hebt demgegenüber die Bemühungen der UNESCO hervor, sich dennoch für eine Welt zu engagieren "in which diverse population exchange ideas, learn cultural practices from one another and produce hybrid forms of culture [...]", während gerade indigene Gruppen auf der nach Eriksen (2001:132) zitierten "archipelago idea of culture" beharren, mit anderen Worten, auf der Idee "the world is made up of cultural islands, each discrete and bounded“ (Kasten 2004:21), um ihre Interessen gegenüber Nationalstaaten und internationalen Institutionen zu vertreten.

\footnotetext{
${ }^{20}$ Weiterführende Literatur zum Phänomen der Globalisierung s. Beck 1998 und zur Kreolisierung s. Hannerz 1987
} 
Beispielhaft für ein, den Leser konfusionierendes Verständnis des Begriffs wäre z.B. die Allgemeine Erklärung zur kulturellen Vielfalt von 2001. In der englischen Fassung der Erklärung heißt es zu Beginn:

"The UNESCO Universal Declaration on Cultural Diversity was adopted unanimously in a most unusual context. It came in the wake of the events of 11 September 2001, and the UNESCO General Conference, which was meeting for its 31st session, was the first ministerial-level meeting to be held after those terrible events. It was an opportunity for States to reaffirm their conviction that intercultural dialogue is the best guarantee of peace and to reject outright the theory of the inevitable clash of cultures and civilizations" (UNESCO 2001:11).

Hier wird Bezug genommen auf das Werk Samuel P. Huntingtons "The clash of civilizations" (Kampf der Kulturen) von 1993. Nach Huntingtons Verständnis stellen Zivilisationen ,"cultural entities" dar, das bedeutet, dass sie sowohl Ganzheiten als auch Einheiten sind, die - laut Huntington - in engem Zusammenhang mit dem Begriff Kultur stehen (vgl. Huntington 1996). Nicht nur, dass die Begriffe von Kultur und Zivilisation in Huntingtons Schrift eine eigentümliche Verwendung finden, auch der Begriff von Kultur wird als eine abgrenzbare objektivierbare und somit statische Einheit betrachtet (vgl. ebd.).

Indem die UNESCO in ihrer Erklärung diese Metapher honoriert, gesteht sie auch deren mögliche lebensweltliche Existenz ein, die es nach Ansicht der UNESCO eben nicht geben sollte und weshalb die Organisation eine Neuformulierung des Kulturkonzepts erwog. Gemäß der Konsensustheorie gelten bestimmte Aussagen dann als wahr, wenn eine potentiell unendlich große Menge von Menschen unter idealen Kommunikationsbedingungen dieser Aussage allgemein zustimmen würde oder mit anderen Worten ausgedrückt: eine semantische Akzeptanz wird zur sozialen Tatsache (vgl. Habermas 1981; vgl. Hetzel 2001). Dies ist meiner Ansicht nach explizit in der Erwähnung des Huntigton'schen Kulturkonzepts zu sehen. Wie ich später noch aufzeige, verwenden rezent viele indigene Gemeinschaften das Bild einer "geschlossenen kulturellen Einheit", um ihren Bedürfnissen und Ansprüchen Geltung verschaffen zu können. Dies ist meiner Ansicht nach zwar nicht zuletzt, aber mutmaßlich auch auf die oben erwähnte internationale Honorierung der Idee einer Kultur als Entität zurückzuführen - eingestehend der Tatsache, dass diese Sicht auch lange Zeit in den Kulturwissenschaften gängig war. 
Die UNESCO bezieht sich also auf eine Perspektive von Kultur, die sie mit ihrer ethnologisch-kulturwissenschaftlichen Definition ad acta legen wollte (vgl. Schöfthaler \&Dyroff 1997:17f). Letztere Definition, die bereits 1982 in Mexiko-Stadt entworfen wurde, wird dann auch gleich in der selben Erklärung rezitiert (2001:12). Man gewinnt den Eindruck, dass die im Bericht „Our creative diversity“von Pèrez de Cuéllar zitierte Aussage Marshall Sahlins:

„,A greal deal of confusion arises in both academic and political discourse when culture in the humanistic sense is not distinguished from „culture“ in its anthropological sense, [...]” (Pérez de Cuéllar 1996: 21; vgl. Sahlins 1994)

keine Beachtung bei der UNESCO fand.

Gerade in politischen Auseinandersetzungen und öffentlichen Diskursen hat der Kulturbegriff im Sinne einer abgrenzbaren Einheit in den letzten Jahren als Erklärung von Unterschieden immer mehr an Bedeutung gewonnen. In diesem Zusammenhang wird der Kulturbegriff gerne von nationalistisch ausgerichteten Interessengruppen missbraucht. Vor allem in Argumentationen, in denen es darum geht, andere Menschen aus der eigenen Gesellschaft auszuschließen. Der Verweis auf kulturelle Unterschiede und unvereinbare Lebensweisen hat den immer stärker diskreditierten Rassebegriff ersetzt (Beer 2003:68; vgl. Kahn 1990:5ff). In solchen, kulturelle Unterschiede betonenden Stellungnahmen wird Kultur, anders als in der Ethnologie, als unveränderbar, homogen und objektivierbar dargestellt (Beer 2003:68). Das heute übliche ethnologische Konzept von Kultur als prinzipiell offen, dynamisches System mit Brüchen und Widersprüchen unterscheidet sich hingegen von diesen gängigen und politisch genutzten Alltagskonzepten (ebd.).

Als eine weitere Kritik zum Pérez de Cuéllar Bericht bemerkt die Autorin MarieTherese Albert, dass sich in der Schrift deutlich euro-amerikanische Einflüsse bemerkbar machen. Sie kritisiert, dass Kultur und Entwicklung in diesem Programm als zwei Seiten eines einzigen Modernisierungsprozesses gefasst und dem Diktat des „westlichen“ Verständnisses von Modernisierung untergeordnet wurden (Albert 2002:31). Für die gegenwärtige Anthropologie/ Ethnologie zumindest fordert Appadurai (1998:13), dass die entscheidende Herausforderung darin bestehe, die kosmopolitisch-kulturellen Formen der modernen Welt zu untersuchen, ohne dabei dem Westen oder einem davon abgeleiteten Modell logische oder chronologische Priorität einzuräumen, was schwerfallen wird, wenn, wie Friedman bemerkt, ,[f]rom the global point of view, culture is a typical product of Western modernity [...]“(1995:80). 
„Das Eigentum bricht einer Furie gleich in das menschliche Herz ein, wälzt die tiefstgewurzelten Gefühle, Ideen und Instinkte um und schafft neue Triebe, Begriffe und Empfindungen“"

\section{Die Anfänge der UNESCO-Konzeptionen des kulturellen Eigentums}

Schon früh begann das Interesse am Schutz von kulturellen Errungenschaften auf der Basis materieller Objekte. Die Vorgeschichte und Entstehung erster Ansätze für eine Konzeption zum Schutze von kulturellem Eigentum reichen weit bis in die Antike zurück (s. Kapitel 2). Ausschlaggebend war dabei meist die Angst einzelner Gesellschaften und Gruppen vor Zerstörung ihrer für sie kulturell bedeutsamen Objekte durch Kriege und Gewalt (vgl. Merryman 2005:11ff).

\subsection{Die Anfänge}

Auf internationaler Ebene wurde kulturelles Eigentum (Cultural Property) als schützenswerte Kategorie erstmalig seit der ersten und zweiten internationalen Friedenskonferenz in Den Haag/ Niederlande von 1899 und 1907 und in der 1907 verabschiedeten „Hague Convention concerning Bombardment by Naval Forces in Time of War" in Teilen behandelt (Frigo 2004:1) und seit den 50'er Jahren durch die UNESCO und anderen „intragovernmental organisations“ weiterentwickelt (Blake 2000:61).

Das weltweit erste Abkommen, das rein und ausschließlich auf den Schutz von kulturellem Eigentum abzielte, war die Haager Konvention zum Schutz von Kulturgut bei bewaffneten Konflikten aus dem Jahre 1954. Die anfängliche Konzeption der UNESCO barg demnach ein eng gefasstes Ziel. Sie entstand als Antwort auf die Zerstörung und den Verlust von bedeutsamen Monumenten und Kunstobjekten während des zweiten Weltkriegs. Blake (2000:61) bemerkt dazu: „It grew out of a feeling that action to prevent their deterioration or destruction was one responsibility of the 
emerging international world order and an element in reconciliation and the prevention of future conflicts".

Bis zum 31.03.2005 sind der Konvention 114 Staaten beigetreten ( UNESCO 2005b; weniger als zur Welterbekonvention, s.u.). Großbritannien und die USA weigern sich bislang, die Konvention zu ratifizieren, obwohl diese Staaten an der Entstehung des Abkommens maßgeblich beteiligt waren und die Prinzipien der Konvention in die nationalen Militärinstruktionen aufnahmen (Prott 1998:222ff; vgl. Strasser 2005:54).

\subsection{Konzeptionelle Erweiterungen und kulturpolitischer Wandel}

Im Jahre 1956 erweiterte die UNESCO ihren Katalog zum Schutz von kulturellem Eigentum, nachdem sie eine Empfehlung über die Handhabung von archäologischen Ausgrabungen annahm (Recommendation on the conduct of archaeological excavations; UNESCO 1956). Der Schutz von archäologischen Funden oblag anfänglich den Staaten, in denen sich die betreffenden Objekte, Monumente oder Kunstwerke befinden und nicht den Staaten, aus denen sie stammten. Die Staaten fungierten als "Treuhänder" eines kulturellen Eigentums, welches für die Menschheit von bedeutsamer Wichtigkeit ist. Neben dem Schutzaspekt galt es auch, die internationale Zusammenarbeit zu fördern und Konflikten präventiv zu begegnen (vgl. Blake 2000). Es folgten weitere UNESCO Empfehlungen auf spezifische Fragen. 1960 wurde die "Recommendation concerning the most Effective Means of Rendering Museums Accessible to Everyone" (UNESCO 1960) angenommen, 1962 die "Recommendation concerning the Safeguarding of the Beauty and Character of Landscape and Sites" (UNESCO 1962) und 1968 die "Recommendation concerning the Preservation of Cultural Property Endangered by Public or Private Works" (UNESCO 1968). Letztere Empfehlung von 1968 hat ihre Entstehung der Rettungskampagne von Abu Simbel ${ }^{21}$ 1959 zu verdanken (Strasser 2005:55).

\footnotetext{
${ }^{21}$ Abu Simbel ist der Name eines Ortes mit zwei berühmten Tempeln in Südägypten, südlich von Assuan. Die Tempel wurden um 1250v.Chr. unter der Regierung von Ramses II. aus einem Sandsteinfelsen geschlagen. Diese Tempel sind die bedeutendsten Denkmäler des antiken Nubiens. Sie waren der westlichen Welt unbekannt, bis sie 1813 von dem Schweizer Forscher Johann Ludwig Burckhardt entdeckt wurden (Scholz 1994:3ff).
} 


\subsubsection{Das Beispiel Abu Simble als materielles unbewegliches Eigentum ${ }^{22}$}

Die Vereinigte Arabische Republik ${ }^{23}$ rief 1959 die UNESCO um Unterstützung bei der Rettung der durch den Bau des Assuan-Staudammes bedrohten Denkmäler an. Es wurde die Internationale Kampagne zur Rettung der Denkmäler von Nubien ${ }^{24}$ ins Leben gerufen. Die Kampagne stand unter der Führung des schwedischen Königs Gustav Adolf VI. Über 40 Staaten kooperierten bei der Kampagne, die die Versetzung der Tempel von Abu Simbel und Philae umfasste. Vor dem Bau des AssuanStaudammes befanden sich in dem heute überfluteten Gebiet u.a. die Tempel von Abu Simbel, die pharaonische Kultstätte von Philae und die Stadt Wadi Halfa (Strasser 2005:55; vgl. Scholz 1994).

Die im 13.Jahrhundert v.Chr. von dem ägyptischen Pharao Ramses II. erbauten Tempel von Abu Simbel wurden in den sechziger Jahren des 20. Jahrhunderts vollständig vor der Zerstörung gerettet. Die Anlagen der Insel Philae, ehemals südlich von Assuan, konnten nur teilweise auf der benachbarten Insel Agilkia erhalten werden. Viele andere historische Bauwerke sind, wie Teile der historischen Landschaft Nubiens, überflutet worden. In einem Großprojekt wurden die Bewohner der nubischen Stadt Wadi Halfa in ein 1200 Kilometer entferntes Gebiet umgesiedelt. Das Unternehmen konnte nach 20 Jahren im Jahre 1980 erfolgreich abgeschlossen werden (ebd. 54). André Malraux, damaliger Kulturminister Frankreichs, äußerte sich wie folgt zu dieser Kampagne: "the first world civilisation publicly proclaims the world's art as its indivisible heritage" (Malraux zitiert in Strasser 2005:54f; vgl. Scholz 1994).

\footnotetext{
${ }^{22}$ Ich übersetze tangible immovable property als materielles unbewegliches Eigentum

${ }^{23}$ Im Februar 1958 stimmten Syrien und Ägypten in Volksabstimmungen mit großer Mehrheit für eine Union der beiden Länder, die unmittelbar darauf unter dem Namen Vereinigte Arabische Republik (VAR) in Kraft trat. Die ägyptische Dominanz innerhalb der VAR sowie das Ausbleiben des erhofften wirtschaftlichen Fortschritts provozierten in Syrien zunehmenden Widerstand gegen die Union mit Ägypten; am 28.September 1961 erhoben sich syrische Offiziere in einem Putsch gegen die Vereinigung mit Ägypten und stellten die Unabhängigkeit Syriens wieder her (Wald 1969: 2ff).

${ }^{24}$ Nubien ist eine Region im Nordosten Afrikas; sie umfasst beide Talseiten des Nils zwischen Assuan in Ägypten und Khartum im Sudan (Scholz 2006:1ff).
} 


\subsubsection{Maßnahmen gegen den internationalen Kunstraub}

Die UNESCO "Convention on the Means of Prohibiting and Preventing the Illicit Import, Export and Transfer of Ownership of Cultural Property" (UNESCO 1970) von 1970 bildet ein weiteres Glied konzeptioneller Ausgestaltung dessen, was kulturelles Eigentum im Sinne der UNESCO ist und was nicht. Die Konvention ist aber auch eine Abkehr von der Empfehlung von 1956 (s.o.). Sie sollte ein wirksames Instrument gegen den illegalen internationalen Kunsthandel sein. Es sollte ein Ausgleich der Interessen zwischen der Nord- und Südhalbkugel bezüglich jenes Kulturgutes geschaffen werden, das aus den ehemaligen Kolonien stammte. Gegenstand des Disputes war und ist jenes Inventar, das in den Museen der ehemaligen Kolonialmächte aufbewahrt wird oder im Zuge archäologischer Ausgrabungen in andere Staaten gelangte. Rückgabeforderungen aus der südlichen Staatenwelt wurden bisweilen mit dem Einwand zurückgewiesen, dass ein optimaler Schutz des betreffenden Kulturgutes eher in den Museen des „Westen“ gewährleistet sei und daher dort verbleibe (Strasser 2005:56).

\subsubsection{Policy-turn der UNESCO}

Während die UNESCO-Konvention von 1954 noch im Trauma des Zweiten Weltkriegs verhaftet war (s.o.) und die Reduzierung bzw. Verhinderung internationaler Konflikte beabsichtigte, so galt es der 1970'er Konvention im Gegensatz zu früheren Empfehlungen und Konventionen dem Selbstverständnis sogenannter „dominierender, entwickelter Staaten“ entgegenzuwirken und die Stellung von „Entwicklungsländern" zu stärken. Die Auffassung der Konvention von dem, was kulturelles Eigentum sei, könnte mit "nationalistisch“ charakterisiert werden, da die Interessen der Herkunftsländer gestärkt wurden (Blake 2000:62) - wohingegen die Empfehlung von 1956, meiner Ansicht nach, mit "kolonialistisch“ umschrieben werden kann. M'Bow, Generaldirektor der UNESCO zu jener Zeit, schreibt in "A plea for the Return of an Irreplaceable Cultural heritage to those who Created It" (1979): 
"The men and women of these [despoiled] countries have the right to recover these cultural assets which are part of their being [...]. The return of a work of art to the country which created it enables a people to recover part of its memory and identity [...]" (UNESCO 1979).

Die Initiative zur Ausarbeitung des Abkommens von 1970 ging von den ehemaligen Kolonialstaaten aus, die große Verluste ihres Kulturgutes beklagten und unter den Folgen von organisierter Plünderung, Diebstahl und Ausverkauf ihrer „materiellen Kultur“ litten (Shyllon 1998:232; vgl. Strasser 2005:56). Die im Kunsthandel führenden Staaten verweigerten die Mitarbeit am Entwurf des Abkommens. Dem Abkommen blieb demnach sein Erfolg versagt, bis im Jahre 1997 Frankreich beitrat und weitere westliche Länder diesem Beispiel folgten (O'Keefe 2000:8f, vgl. Strasser 2005:56f). Um den Interessenaustausch und die Kulturgutrestitution zwischen den Staaten zu forcieren, setzte die Generalkonferenz der UNESCO 1978 ein zwischenstaatliches Komitee ${ }^{25}$ ein, das sich seither alle zwei Jahre bei seinen Treffen um den Nord-Süddialog bemüht (vgl. Strasser 2005:56; Prott\&O’Keefe1989:1560f). Zusätzlich rief die UNESCO 1999 mit ihrem „Dealers Code،26 Kunsthändler, Sammler und Museen zur Mithilfe und Umsicht auf. Dieses Abkommen regelt aber nur das Rückgabeverfahren zwischen Staaten. Privatpersonen können aus dem Abkommen keine unmittelbaren Ansprüche erheben. Um diesem Defizit entgegenzuwirken, übertrug die UNESCO der UNIDROIT ${ }^{27}$ in Rom den Auftrag zur Ausarbeitung einer Konvention über gestohlene oder rechtswidrig ausgeführte Kulturgüter ${ }^{28}$, die 1995 in Kraft trat.

\footnotetext{
${ }^{25}$ Intergovernmental Committee for Promoting the Return of Cultural Property to its Countries of Origin or its Restitution in Case of Illicit Appropriation. Das Komitee wurde durch die UNESCO Resolution No. 4/7.6/5 (1978) in Leben gerufen (UNESCO 1978b).

${ }^{26}$ Die vollständige Bezeichnung lautet: International Code of Ethics for Dealers in Cultural Property (UNESCO 1999).

${ }^{27}$ Die UNIDROIT (International Institute for the Unification of Private Law/ Internationales Institut für Rechtsvergleichung) ist eine spezielles Institut der UN, das sich um die Belange kümmert, die in der UNIDROIT Konvention von 1995 festgehalten sind.

${ }^{28}$ UNIDROIT Convention on Stolen or Illegally Exported Cultural Objects von 1995.
} 


\subsubsection{Die Entwicklungen seit der Welterbekonvention von 1972}

1972 verabschiedete die UNESCO die "Convention concerning the Protection of the World Cultural and Natural Heritage" (Welterbekonvention). Mit der Welterbekonvention vollzog die UNESCO einen konzeptionellen Wandel. War es bis dahin kulturelles Eigentum, das als Leitkategorie und Konzept galt, so wurde nun das Konzept des Kultur- und Naturerbes betont (s.Kapitel 6\&7). Die Welterbekonvention kann für sich in Anspruch nehmen, weltweit das Bewusstsein über kulturelles Eigentum und vor allem kulturelles Erbe gefördert zu haben. Andererseits hat das Prestige, das von den Welterbestätten ausgeht, aber auch dazu beigetragen, dass kulturelles Eigentum bzw. kulturelles Erbe vermehrt zur Zielscheibe politisch motivierter Angriffe oder gar Zerstörungen wurde (Strasser 2005:63, vgl. O’Keefe 1992; vgl. Blake 2000).

Die zunehmende Popularität von kulturellem Eigentum und kulturellem Erbe führte in den letzten Jahrzehnten dazu, dass die internationale Staatengemeinschaft eine zunehmende Instrumentalisierung von kulturellem Eigentum bei bewaffneten Auseinandersetzungen als politisches Faustpfand und als militärisches Ziel - z.B. die Zerstörung der Buddha-Statuen von Bamiyan/ Afghanistan 2001 durch die Taliban oder die vorsätzliche Beschießung der so genannten „Perle der Adria“, Dubrovnik/ Kroatien während des jugoslawischen Bürgerkriegs von 1991 - feststellte (Strasser 2005:62).

Da die erste Haager Konvention von 1954 noch auf den konventionellen Kriegsfall abzielte, d.h. der Schutz war bei "klassischen" kriegerischen Handlungen zwischen Staaten anzuwenden, galt aber nicht bei internen Konflikten, Bürgerkrieg und Terrorakten, und keinen hinreichenden Schutz auf Grund der neueren Entwicklungen gewährleistete, so trugen die neueren Ereignisse zu einer Revision der Konvention von 1954 bei. Im Jahre 1999 wurde auf Grund dieser konzeptionellen Mängel das „Zweite Zusatzprotokoll zur Haager Konvention von 1954" beschlossen und 2004 in Kraft gesetzt. Bedeutend daran ist nicht nur, dass das Protokoll erneut kulturelles Eigentum hervorhebt (im Gegensatz zur Welterbekonvention), sondern erstmalig die Instrumentalisierung von kulturellem Eigentum in Kriegszeiten als politisches Pfand in den Status von Kriegsverbrechen erhebt. Taten dieser Art werden von nun an als international geächtet und als Kriegsverbrechen betrachtet und durch den Internationalen Strafgerichtshof in Den Haag strafrechtlich verfolgt. Das zweite Zusatzprotokoll von Den Haag umfasste einige Neuerungen; z.B. können Einzelpersonen zur straf- 
rechtlichen Verantwortung gezogen werden. Jeder Vertragsstaat hat, laut Artikel 28 der Konvention, die Möglichkeit, eine Person jeglicher Nationalität wegen eines Vergehens gegen die Konvention mittels des eigenen Rechtssystems zur Verantwortung zu ziehen (Hladig 2003:47). Des weiteren gibt es die Einführung eines Fonds, eine Anleitung zu Vorbereitungsmaßnahmen in Zeiten des Friedens und eine verbesserte Begriffsbestimmung (vgl. Strasser:62f, vgl. Hladig 2003:44-49).

\subsection{Meum et Tuum}

Das Konzept eines kulturellen Eigentums erfasst zweifellos eine große Anzahl an Manifestationen des kulturellen Schaffens und der Kreativität der Menschheit (s.u.). In diesem Kapitel behandle ich ausschließlich die Frage, was kulturelles Eigentum im Gegensatz zu kulturellem Erbe auf der konzeptionellen Ebene der UNESCO darstellt und wie sich kulturelles Eigentum aus ethnologisch-kulturwissenschaftlicher Perspektive konstruieren lässt.

Unser heutiges Alltagsverständnis von Eigentum beruht oft auf einer unbewussten Dynamik - einer tiefen Resonanz von Mein und Dein. Diese Dichotomie durchdringt und bestimmt auf eine triviale Weise unser Denken und Handeln in Bezug auf Eigentums- und Besitzverhältnisse. Um es mit Gray's Worten zu sagen: „Irrespective of the precise language chosen to serve as the doctrinal vehicle, the major inarticulate premise in each of these areas is generated by some underlying half-conscious perception of property" (1991:306). Eine besondere Begebenheit dabei ist, folgt man Gray, die Tatsache, dass Eigentumsvorstellungen (property notions) abhängig sind von den jeweiligen Gesellschaften, in denen sie Geltung besitzen (ebd.).

In den folgenden Abschnitten werde ich ausgehend von einer kurzen Übersicht über die Begriffsgeschichte und dem in Mitteleuropa gängigen Verständnis von Eigentum in Abgrenzung zu Besitz, anschließend ethnologisch-kulturwissenschaftliche Perspektiven von Eigentum skizzieren, um sodann die qualitative Erweiterung hin zu kulturellem Eigentum der UNESCO Perspektive gegenüberzustellen und abschlieBend zu diskutieren. 


\subsubsection{Eigentum und Besitz}

In unserem allgemeinen Sprachgebrauch und alltäglichem Verständnis verwenden wir die Begriffe Eigentum und Besitz meist gleichbedeutend und im willkürlichen Wechsel. Bei genauer Betrachtung verhalten sich die Bedeutungen hinter den Begriffen je nach Kontext, in dem sie Verwendung finden aber weitaus differenzierter.

Ausgehend von einer sprachgeschichtlichen Genese der Begriffe lässt sich aufzeigen, dass sich das Substantiv „Eigentum“ vom althochdeutschen egendom als Glosse für lat. hereditas, possessio (Ritter 1971:339) und vom mittelhochdeutschen Wort eigentuom ableitet, das im weitesten Sinne "freies Besitzrecht" bedeutete (Wermke 2001:172). Das substantivierte oder adjektivisch verwendete Partizip „eigen“ (ahd. eigan), aus dem die Substantive „Eigenschaft“ und „Eigentum“ abgeleitet sind, steht für die archetypische Zuordnungsbeziehung Subjekt-Objekt. Der Eigentumsbegriff ist in der Lage, immer neue Sinngehalte in sich aufzunehmen, sofern das Muster des Subjekt-Objektverhältnisses ${ }^{29}$ vorhanden ist (Schwab 1975:65). Dieser Sachverhalt erklärt, nach Ansicht Schwabs, die Mehrheit der Eigentumsbegriffe bis heute (ebd.). Dem außerwissenschaftlichen Eigentumsbegriff stehen wissenschaftliche Termini gegenüber, etwa der juristische, der ökonomische und auch der ethnologische, die notwendig wiederum einen unterschiedlichen Sinngehalt aufweisen. Der Eigentumsbegriff wird umso veränderlicher, je stärker er in den Bann von Staats- und Gesellschaftsdoktrinen gerät und folglich in seiner Bedeutung systemabhängig wird (Schwab 1975:65). Für den Eigentumsbegriff bedeutet dies, dass die verschiedensten Variationen liberaler, sozialer, christlicher oder sozialistischer Eigentumslehren darauf angewiesen sind, inwieweit sie sich in den diversen Rechtsordnungen ihrer jeweiligen Gesellschaft verwirklichen und eine Veränderung der Eigentumsgestalt bewirken (ebd. 103) ${ }^{30}$. Hann äußert sich zu der komplizierten und komplexen Ge-

\footnotetext{
${ }^{29}$ Es kann bedauerlicherweise nicht nachvollzogen werden, was genau der Begriff des Objektes bei Schwab umfassen soll und kann. Man bedenke aber die Schwierigkeiten, die sich ergeben, wenn geistiges Eigentum oder das Phänomen der Sklaverei in eine Subjekt-Objektbeziehung gebracht würden.

${ }^{30}$ Im Umfang dieser Arbeit kann ich leider nicht auf die geschichtliche Genese des rezent in Deutschland verwendeten gemeinrechtlichen Eigentumsbegriffs eingehen. Eine ausführliche Darstellung seiner Transformation vom Mittelalter ausgehend bis heute findet sich bei Brunner 1975. Es sei aber angemerkt, dass beim Eigentumsbegriff überwiegend das Subjekt-Objektverhältnis bestimmend für die unterschiedlichen Sinngehalte war und ist, im Gegensatz zum Kulturbegriff, dessen Bedeutungsgehalt sich mit den Epochen grundlegend wandelte (s. Kapitel 4).
} 
schichte der Eigentumskonzeptionen in dem Sinn, dass kein Forscher oder Forschergruppen, die auch nur das Wort Eigentum in ihrem Titel haben, eben diese Komplexität außer Acht lassen können (Hann 2005:2). Ich verweise in diesem Zusammenhang gerne auf die Möglichkeit - um einer wahrhaft ausführlichen Analyse der Genese des Phänomens Eigentum gerecht zu werden - sprichwörtlich bei Adam und Eva anzufangen, die ja bekanntermaßen wegen „Eigentumsvergehen“ aus dem Paradiese vertrieben wurden.

Seriöserweise sind in der Geschichte Europas grundsätzlich zwei Perspektiven hervorzuheben, die unsere alltägliche Auffassung von Eigentum und Besitz geprägt haben. Auf der einen Seite steht das heute global dominante Paradigma und ideologische Prinzip eines liberalen Eigentumsbegriffs, das auf die Arbeiten und das Wirken Adam Smith zurückzuführen ist (Hann 1998a:1; vgl. Hann 2004:124). Das Paradigma wird bestimmt durch diverse Schlüsselideen wie freie Märkte, Pluralismus, der Hervorhebung der Stellung des Individuums und vor allem des Privateigentums (Hann 1998a:1). Auf der anderen Seite der Dichotomie steht das Verständnis eines kollektiven Eigentums, gestützt durch die ideologischen Lehren des Kommunismus und Sozialismus, in denen privates Eigentum eingeschränkt und stigmatisiert wird, während kollektives Eigentum entsprechend privilegiert ist (Hann 2004:126).

Der Begriff „Besitz“ als Substantiv tritt erstmals seit dem 15. Jahrhundert im Mittelhochdeutschen in Form von besez auf und stand in der Bedeutung „dessen was man besitzt, worüber man verfügt" (Wermke 2001:772). Abgeleitet hat sich das Wort aus der Präfixbildung „besitzen“, das wiederum aus dem Verb „sitzen“ gebildet wurde. „Besitzen“ (got. bisitan, ahd. bisizzan, mhd. besitzen) bedeutete ursprünglich „um etwas sitzen“, dann „sich auf etwas setzen; etwas in Besitz nehmen, haben“ (Wermke 2001:172; Mackensen 1985:70).

Die für uns als Gesellschaftsmitglieder gemeinrechtlich relevantesten Definitionen von Eigentum und Besitz finden sich in den Gesetzbüchern. Eigentum, im Sinne eines verfassungsmäßigen Grundrechts wird grundlegend durch Artikel 14 des Grundgesetzes der Bundesrepublik Deutschland garantiert (Artikel 14 GG) ${ }^{31}$. „Das Eigentum“ wird durch das Bundesverfassungsgericht bestätigt als ,[...] ein elementa-

\footnotetext{
${ }^{31}$ Ich gehe hier nicht weiter auf die verfassungsrechtlichen Einzelheiten, wie die grundgesetzlich bestimmte Sozialbindung des Eigentums ( $\$ 14$ Abs. 2 GG) und die ebenfalls grundgesetzlich garantierte Möglichkeit der Enteignung ( $\$ 14$ Abs.3 GG) zum Wohle der Allgemeinheit ein. Im Rahmen dieser Arbeit wird nur das gemeinrechtliche Grundverständnis unserer Gesellschaft von Nöten erachtet, um darauf aufbauend ethnologische Abstraktionen und das Eigentumsverständnis der UNESCO zu kontrastieren.
} 
res Grundrecht, das in einem inneren Zusammenhang mit der Garantie persönlicher Freiheit steht“" (BVerfGE 24.389; vgl. Wesel 1985:95). In der Sprache und dem Verständnis der Juristen ist Eigentum „die Beziehung einer Person zu einer Sache im Sinne einer absoluten Beherrschung“ (Baur 1983:222; vgl. Wesel 1985:96). Der Begriff des „Absoluten“ impliziert, dass es im Prinzip nicht um die Beherrschung der Sache geht, sondern vielmehr um den Ausschluss, die Exklusion des Anderen (Wesel 1983:96; vgl. Gray 1991). Die Grenzen dessen, was Eigentum bedeutet, liegen nach Ansicht Grays ,"not by the „thinglikeness” [sic.] of particular resources but by the physical, legal and moral criteria of excludability"' (Gray 1991:299).

Im dritten Bürgerlichen Gesetzbuch (BGB) wird in Abschnitt 3 des Sachenrechts der Inhalt des materiellen Eigentums unter $\S 903$ wie folgt definiert: „Der Eigentümer einer Sache kann, soweit nicht das Gesetz oder Rechte Dritter entgegenstehen, mit der Sache nach Belieben verfahren und andere von jeder Einwirkung ausschließen“ $(\$ 903 \mathrm{BGB})^{32}$. Der Begriff des Besitzes hingegen wird im dritten Buch des BGB unter §854 des Sachenrechts definiert: „Der Besitz einer Sache wird durch die Erlangung der tatsächlichen Gewalt über die Sache erworben“ (§854 BGB).

Eigentum wird im BGB als rechtlich abgesicherte Verfügungsgewalt über materielle Güter durch Individuen oder Gruppen deklariert, wohingegen Besitz als die „tatsächliche Herrschaft“ einer Person über eine Sache ausgelegt ist (vgl. Haller 2005:163). Als Bespiel wäre zu erwähnen, dass sich der deutsche Personalausweis im Besitz seiner Inhaber befindet, deren Verfügungsgewalt aber durch Auflagen eingeschränkt wird, da der Staat Eigentümer der Ausweise ist (ebd.).

Bedeutend ist die Erweiterung der Begriffe Eigentum und Besitz auf immaterielle Elemente. Geistiges Eigentum bezieht sich auf durch den Staat gewährte, Exklusivrechte auf immaterielle Sachverhalte. Dabei werden jedem, außer dem Rechteinhaber oder Lizenznehmer zeitlich begrenzte Verbote bezüglich Verwendung Nachahmung oder Kopie auferlegt (Bauer 2006:1f; vgl. Bell-Krannhals 1990:308f; vgl. Ramsauer 2004 ).

Folgende sehr unterschiedliche und miteinander konkurrierende Rechte werden unter geistigem Eigentum vereinigt: das Urheberrecht, Patente; Handelsmarken, Handelsnamen oder Phrasen, geschütztes Design und Geschäftsgeheimnisse. Der

\footnotetext{
${ }^{32}$ Der Begriff der Sache wird im BGB Abschnitt 2 §90 definiert und umfasst nicht immaterielles Eigentum. Die juristischen Definitionen von Eigentum und Besitz im BGB umfassen somit nur materielles Eigentum.
} 
Begriff des geistigen "Eigentums" hat, wie oben dargelegt, sehr wenig mit dem klassischen Eigentumsbegriff zu tun (Bauer 2006:1f; vgl. Ramsauer 2004).

Eigentum im klassischen oben dargestellten Sinn bedeutet absolute Verfügungsgewalt über einen konkreten Gegenstand. Nicht mehr und nicht weniger. Ein Haus oder ein Auto sein eigen nennen heißt nicht, dass man jedem anderen Menschen den Besitz (hier im Sinne von Eigentum) eines identischen Hauses oder Autos verwehren kann (selbst wenn der Plan gestohlen wurde oder das Haus nachgebaut wurde, kann man höchstens Schadenersatz verlangen, aber nicht, dass der andere sein Haus einreißt). Dagegen ist z.B. ein Patent ein staatlich gewährtes Monopol z.B. auf eine Idee oder ein Verfahren. Wenn jemand z.B. eine Idee oder ein Verfahren besitzt, darf jemand, der unabhängig auf die gleiche Idee kommt oder dasselbe Verfahren ausarbeitet, dieses nicht kommerziell verwenden (Bauer 2006:1f).

Von einem einheitlichen Eigentumsbegriff kann also nicht die Rede sein. Übereinstimmung besteht nach Ansicht Schwabs allenfalls in der Mobilität des Begriffs (1975:114).

\subsubsection{Ethnologische Perspektiven auf Eigentum und Besitz}

Theoretische Reflexionen und empirische Forschungen zum Themenkomplex Besitz und Eigentum gab es in der Ethnologie bereits seit ihrer Begründung als wissenschaftliche Disziplin im 19 Jahrhundert $^{33}$ (Hann 2004:125). Es war und ist die Aufgabe der Ethnologie, den Wert von Eigentum und Besitz in Bezug auf die jeweilige untersuchte Gesellschaft zu definieren und möglichst getrennt von eigenen Vorstellungen zu präsentieren (Bell-Krannhals 1990:308). In allen Gesellschaften gibt es Eigentumsrelationen (Hann 1998a:6; vgl. Bell-Krannhals 1990; vgl. Nippold 1954). Eigentum und Besitz sind universale Phänomene. Überall wird etwas als eigen besessen, selbst in den materiell am bescheidensten ausgerüsteten Gemeinschaften (Bell-Krannhals 1990:309; vgl. Hann 1998a; vgl. Wesel 1985; vgl. Nippold 1954), deren Eigentumskonzeptionen sich durchaus als komplex erweisen können (Hann 2004:127). Zudem kommt, dass zu jeder Zeit und in jedem kulturellen Kontext Eigen-

\footnotetext{
${ }^{33}$ Eine ausführliche Übersicht über die Geschichte der Eigentumskonzeptionen in der Ethnologie gibt Hann 1998a.
} 
tums- und Besitzkonzeptionen abhängig sind von diversen Objektkategorien, z.B. ob ihre Beschaffenheit materieller oder geistiger Art und Weise ist (Hann 1998a:3).

Wie oben bereits dargestellt, wird der Eigentums und Besitzbegriff in unserer heutigen Zeit überwiegend auf "Dinge“ bezogen oder auf ökonomisch interessante Facetten des menschlichen Daseins (s.u.). Folgt man den Aussagen Hanns so ist ,,[...] in established Western theoretical and academic usage property [...] not an activity or an thing at all, but the rights that people hold over things which guarantee them a future ,income stream"“(1998a:4).

Eigentum und Besitz sollten laut Hann konsequenterweise als Resultat sozialer Beziehungen verstanden werden (1998a:4). Als soziale Phänomene können Eigentum und Besitz dadurch auf dem Hintergrund ihrer jeweiligen sozialen Organisationsprinzipien verstanden werden. Schott äußert sich dazu wie folgt:

„Die Formen des Eigentums hängen also weitgehend von seinen Funktionen in der jeweiligen Kultur und Sozialordnung ab, ohne dass freilich der Grundgedanke einer wie immer gearteten Sachherrschaft von Individuen und von Gruppen bei irgendeinem Volke ganz fehle. Ohne die Zuordnung des Besitzes unter die rechtliche Herrschaft von Personen oder Gruppen bestünde ein Zustand der Anarchie und Desintegration bei den sogenannten Naturvölkern, wie wir ihn nirgendwo beobachten können. Tatsächlich nimmt also das Eigentum (im weitesten Sinne) in jeder Kultur einen funktionell unentbehrlichen Platz ein und ist aus dem Kultur- und Sozialgefüge gar nicht wegzudenken“ (1970:116; zitiert in Bell-Krannhals 1990:309)

Eine in diesem Kontext vorgelegte Definition von Hoebel lautet:

"The essential nature of property is to be found in social relations rather than in any inherent attributes of the thing or object that we call property. Property, in other words, is not a thing, but a network of social relations that governs the conduct of people with respect to the use and disposition of things" (1966:424; zitiert in Hann 1998a:4).

Als Beispiel hierzu wäre Davis Aussage anzuführen: "you cannot sue an acre; a boundary dispute is not a dispute with a boundary. [...] The study of property rules in general [...] is the study of relationships between people" (1973:157; vgl. Hann 1998a:5) und um es mit Maurice Godelier zu betonen: "a form of property only exists when it serves as a rule for the concrete appropriation of reality. Property only exists when it is rendered effective in and through a process of concrete appropriation" (1986:81). 
Wie oben angemerkt existiert im „westlichen“ Kontext hauptsächlich die Dichotomie zwischen Individual- und Kommunaleigentum mit einer Dominanz des liberalen $\mathrm{Pa}$ radigmas (Hann 1998a: 1f; 7; vgl. Hann 2004:124f). Alle Gesellschaften haben zwar Eigentumsbeziehungen und -vorstellungen, aber nicht notwendigerweise in dem oben angemerkten Sinne. Für eine bessere Analyse möglicher Begebenheiten wäre es nach Meinung Hann's notwendig, ein Kontinuum von „Individual“ (Privat) über „Familiär" hin zu Formen der „Kooperation“ und/ oder „Korporation“ bis schließlich zu „Kommunalen“ (Publik) Formen zu konstruieren (Hann 1998a:6f; vgl. Hann 2004; vgl. Bell-Krannhals 1990). Hann (2004:126) führt als Beispiel an, dass „, d] Gartenbau betreibenden Gesellschaften Eigentum des Königs oder Häuptlings sein [kann], die eigentliche Zuteilung und den Zugang zu Land [...] aber üblicherweise rangniedere Anführer wie der Dorfvorsteher festlegen und unkultiviertes Land wird meist als Gemeineigentum (Allmende) betrachtet, was nicht bedeutet, dass es offen zugänglich sein muss".

Um eine ethnozentrische Zweiteilung in Privat- und Kommunaleigentum zu überwinden und Eigentum als allgemeinen Vergleichsbegriff anzuwenden (Hann 2004:127), haben die Rechtethnologen Franz und Keebet von Benda-Beckmann und Melanie Wiber ${ }^{34}$ ein entsprechendes Modell entwickelt. Das Modell unterscheidet zwischen einer kulturell-ideologischen Ebene und einer Ebene der gesetzlichen Regulierung. Was auf ideologischer Ebene hervorgehoben wird, z.B. die absolute Verfügungsgewalt oder die enge Beziehung zur Religion, wird nicht unbedingt der rechtlichen Institutionalisierung von Eigentum entsprechen. Konkrete soziale Beziehungen bilden eine dritte Ebene, während eine vierte Ebene Eigentumspraktiken umfasst, die in wechselseitigen Beziehungen zu den gesellschaftlichen Ausdrucksformen von Eigentum auf den anderen drei Ebenen stehen (Hann 2004:127; vgl. Hann 2005:4). Dieses Modell trägt nach Ansicht Hann's (2004:127) sowohl dem Pluralismus unterschiedlicher Eigentumskonstruktionen wie auch deren Multifunktionalität Rechnung. BendaBeckmann und Wiber definieren Eigentum dementsprechend:

„Property in the most general sense concerns the way in which the relation between society's members with respect to valuables are given meaning, form, and significance. Property in this analytical sense is not one specific type of right or relation such as ownership but a cover term encompassing a wide variety of different arrangements, in

\footnotetext{
${ }^{34}$ Das in Hann 2005 zitierte Werk der Benda-Beckmanns und Wiber ist bis dato noch nicht erschienen. Ich kann daher kein Erscheinungsjahr im Literaturnachweis angeben.
} 
different societies, and across different historical periods (Benda-Beckmann\&Wiber in Hann 2005:4).

Eine weitere Facette von Eigentum ist, dass "[...] property can never be separated either from economic production or politico-ideological power: Its absolutely central position within any mode of production derives from its linkage of the two, which in precapitalist social formations becomes an outright and official fusion" (Anderson 1979:404f; vgl. Hann 1998a:47). Eigentum kann somit als Knotenpunkt zwischen Recht und Ökonomie verstanden werden, der, wie Hann es beschreibt: „[is] linking the two in all social formations" (Hann 1998a:46).

Da Eigentum nun mehr als Schnittstelle zwischen Recht und Wirtschaft hervortritt, muss betont werden, dass gerade das liberale Paradigma des Eigentumsbegriffs in den letzten Jahrzehnten eine globale Verbreitung gefunden hat (Hann 1998a:1; vgl. Appadurai 1986). Hierbei ist die Beziehung zwischen Menschen und ihren Objekten in ihrer sozialen Umwelt evident, da die Subjekt-Objekt-Beziehung auch eine Rolle bei der Konstruktion sozialer Identität spielt (Hann 1998a:2). Das Modell des Privateigentums sei, laut Hann, dabei global vorherrschend (ebd.). Hann äußert sich wie folgt: „A standardized model of private, exclusive ownership has now been disseminated to most societies, including in recent years to the former communist societies [...]“ (1998a:1). Eine Frage, die sich dabei für die Ethnologie stellt, ist, wie "Dinge“ konsumiert werden, auch in Bezug auf die Bildung von Identitäten, ob als Individuum oder im weiteren sozialen Kontext. Ein Schlüssel-Konzept ist die Betrachtung des Wertes der "Dinge" an sich. Bedeutend wäre die Dokumentation und Erkundung der Präferenzen, die ökonomische Aktionen erzeugen (Hann 1998a:31; vgl. Appadurai 1989; vgl. Benda-Beckmann\&Wiber in Hann 2005:4).

Es lässt sich also festhalten, dass Eigentum und Besitz in ihrem sozialen Kontext universale Phänomene sind (vgl. Bell-Krannhals 1990; vgl. Hann 1998a), resultierend aus unterschiedlichen Machtverhältnissen und Zuordnungsprinzipien (vgl. Schott 1970), bzw. rechtlicher Herrschaft und sozialen Beziehungen (vgl. Hoebel 1966), die soziale Identitäten schaffen (vgl. Hann 1998a; vgl. Appadurai 1989), in denen verschiedene Akteure den „Dingen“ Bedeutungen und symbolische Kodes mit unterschiedlichen Zielen und Absichten beimessen (vgl. Benda-Beckmann\&Wiber in Hann 2005), und die als Schnittstelle zwischen Recht und Wirtschaft interpretierbar sind (Anderson 1979:404f; vgl. Hann 1998a:47). 
Was sind das für Machtverhältnisse? Wer sind die Akteure? Wie sind die Machtverhaltverhältnisse verteilt und welche Akteure sind bei diesen Prozessen beteiligt? Was ist dann kulturelles Eigentum und kulturelles Erbe in analytischer Abgrenzung zu Eigentum oder Erbe per se? Wer erzeugt und wandelt die symbolischen Kodes und Bedeutungen, die den „Dingen“, die als Eigentum deklariert werden, inne wohnen und welche Bedeutung hat in diesem Zusammenhang die Kulturpolitik der UNESCO? Von Interesse dürfte auch die Frage sein, wer profitiert, wenn Eigentum zwischen Recht und Ökonomie angesiedelt ist und das liberale Paradigma global vorherrscht?

\subsubsection{Kulturelles Eigentum und die UNESCO}

Mit der adjektivischen Erweiterung „kulturell“ wird dem Substantiv „Eigentum“ eine besondere Eigenschaft bzw. Qualität zugeordnet. Was genau ist dieses kulturelle Eigentum und welche Qualität besitzt es in Abgrenzung zu „normalem“ Eigentum? Allgemein muss angemerkt werden, dass im Fall von kulturellem Eigentum wie auch bei kulturellem Erbe die verschiedenen linguistischen Übersetzungen der termini technici keine Gleichwertigkeit besitzen, was dazu führt, dass es auch keine einheitliche Konzeption gibt. Dies trifft besonders dann zu, wenn kulturelles Eigentum in der linguistischen Form von Cultural Property auf internationaler Ebene und in rechtsverbindlichen Texten übersetzt wird als: biens culturels (franz.), bienes culturales (span.) oder als Kulturgut. Dies ist offensichtlich keine äquivalente Übersetzung in eine andere Sprache, was ebenfalls zu der Annahme führt, dass es zwar geringfügige, aber signifikante Abweichungen zwischen nationalen, nationalen und internationalen, wie auch zwischen den einzelnen internationalen Konzeptionen der UNESCO von "Cultural Property“ gibt (Frigo 2004:2). Frigo bemerkt: „, Here it must be stressed that there is no universally shared definition of either "cultural heritage“ (,patrimonio culturale“, „patrimoine culturel") or of „cultural property“ (,,beni culturali“, „,biens culturels"), as each multilateral agreement gives its own definition of those concepts in order to determine the specific scope of application of the relevant rules"' (2004:5).

Was genau ist dann als Kern eines kulturellen Eigentums im Sinne der UNESCO zu betrachten? Welche Gemeinsamkeiten haben die unterschiedlichen internationalen Konzepte der UNESCO, dass sie als "einheitlich“ interpretierbar wären? Wie bereits Frigo (2004:2) bemerken auch O'Keefe und Prott (1984:8): „[...] for various reasons each Convention and Recommendation has a definition drafted for the purposes of 
that instrument alone; it may not, at this stage be possible to achieve a general definition suitable for use in a variety of contexts". 35

Viele Autoren, die sich aus kulturwissenschaftlich-ethnologischer Perspektive mit kulturellem Eigentum beschäftigen, sehen dieses als eine Subsumtion oder als ein Teil eines jeweilig kulturellen Erbes. Brown z.B. konzeptionalisiert „[...] cultural heritage as a set of things and practices subject to principles of group ownership - in effect, as a form of property [...]" (2004:50) und Kasten definiert kulturelles Eigentum in einem übergreifenden Zusammenhang als:

"The knowledge that gives meaning to practices, objects and land is often specific to, or associated with, particular groups. Transmitted across generations, it is regarded in many societies as cultural heritage to which members can assert privileged claims. Such knowledge is often displayed and transferred as cultural property through symbols, as a means of communicating social networks and for securing a ceremonial dialogue with the supernatural. As a shared cultural tradition, it is essential for the identity of a group" (2004:9; vgl. Ebd. 2002:2) ${ }^{36}$.

Die Definition von Kasten umfasst in ihrer Prozesshaftigkeit sowohl eine - meiner Meinung nach unerlässliche - Definition von Kultur, von kulturellem Erbe und kulturellem Eigentum. Der Autor fasst diesen Prozess im Zusammenhang mit einer geteilten Tradition innerhalb eines kulturellen Kontextes (vgl. ebd. 2004; vgl. ebd. 2002).

Thompson hingegen ist der Ansicht, dass kulturelles Eigentum sich wesentlich durch den inm beigemessenen Wert auszeichnet, „[...] and the value attributed to a given object is itself a cultural expression that often changes over time" (vgl. Thompson 1979 ; zitiert aus Merryman 2005:33). Es stellt sich dabei natürlich die Frage, ob die Symbolisierung von Objekten, wie in der Definition Kastens oder der in Thompsons Definition erwähnte beigemessene Wert implizit nicht das Gleiche darstellen? Welches ist das Resultat des anderen oder in welchem Zusammenhang wären beide zu betrachten? Und wenn Brown kulturelles Eigentum als eine Form des kulturellen

\footnotetext{
${ }^{35}$ Es ist nicht die Aufgabe dieser Arbeit eine generelle Definition zu erarbeiten, sondern die UNESCOKonzeptionen aus ethnologischer Perspektive zu hinterfragen.

${ }^{36}$ Auf die Abstraktion und Bedeutung von kulturellem Erbe und dem Begriff der Tradition wird noch in einem späteren Kapitel einzugehen sein. Ich betrachte hier ausschließlich das Phänomen eines kulturellen Eigentums in Kontrastierung zur Konzeption der UNESCO. Ein Problem bei der Betrachtung von kulturellem Eigentum als eigenständige Abstraktion in Abgrenzung zu kulturellem Erbe, Tradition und einem Kulturbegriff besteht meines Erachtens nach in der nicht verfügbaren Literatur bzw. darin dass die meisten Autoren, wie auch die UNESCO, die Begriffe uneinheitlich und nicht deutlich voneinander abgegrenzt definieren und verwenden.
} 
Erbes definiert und einer jeweiligen Gruppe die Eigentumsrechte daran zuspricht, muss man sich fragen, welchen Stellenwert z.B. Sprache hat und welche Rechte sich für diejenigen Gruppen ergeben, die maßgeblich an der Geschichte und der Identität der Anderen beteiligt waren?

Sowohl Kasten, wie auch Brown und Thompson verfassen kulturelles Eigentum implizit und explizit durch ein diachrones Element. Zudem verweisen Brown und Kasten auf die Besonderheit, dass kulturelles Eigentum primär das Resultat von Beziehungen innerhalb einer bestimmten Gruppe ist, die sich als eine ethnische oder kulturelle Einheit konstruiert bzw. konstruiert wird (vgl. Brown 2004:51; vgl. Kasten 2004:21). Ein weiteres Merkmal von kulturellem Eigentum besteht in der identitätsstiftenden Bedeutung und dem Wert, den es für eine Gruppe von Menschen hat (vgl. Brown 2004:49; vgl. Kasten 2004:9; vgl. Thompson 1979; vgl. Strathern 1996:30 zitiert in Brown 2004:49). Merryman hingegen kommentiert den Versuch einer Definition von kulturellem Eigentum mit der Feststellung, dass die Kategorie des kulturellen Eigentums heterogen, amorph und grenzenlos sei (2005:11f).

In Abgrenzung zu Eigentum per se bedingt sich kulturelles Eigentum somit durch seine identitätsstiftende Bedeutung für eine spezifische Gruppe von Menschen, die einer materiellen oder geistigen Tatsache durch die Zeit hindurch und durch soziale Beziehungen Bedeutung beimessen und dies im Sinne eines „Eigentums“ fassen.

In welchem Kontrast stehen demgegenüber die UNESCO-Konzeptionen von kulturellem Eigentum?

Obwohl Frigo (2004:5), wie auch Prott und O'Keefe (1984:8), bemerkt, dass es keine einheitliche Konzeption von kulturellem Eigentum bei der UNESCO gibt, so lassen sich meiner Ansicht nach zumindest einige Grundannahmen feststellen, die sinngemäß und implizit in den diversen Konventionen und Empfehlungen der UNESCO im Bezug auf kulturelles Eigentum vorhanden sind.

Kulturelles Eigentum ist im Sinne der oben genannten Definition Kastens also eine Manifestation von kulturellem Wissen in Form von Symbolen, die ihre Bedeutungen durch soziale Kommunikation innerhalb und zwischen Netzwerke und Generationen erhalten (vgl. Kasten 2004:9; vgl. Kasten 2002:2).

Die UNESCO definiert demgegenüber kulturelles Eigentum in ihren unterschiedlichen Konventionen und Empfehlungen (vgl. u.a. UNESCO 1954; UNESCO 1956; UNESCO 1960; UNESCO 1976; UNESCO 1968; UNESCO 1999) als eine Addition von ästhetischen und somit materiellen Elementen im Interesse und mit besonderer 
Bedeutung für die gesamte Menschheit mit der qualitativen Grundlage eines statischen Kulturverständnisses (vgl. UNESCO 1954) oder als Angelegenheit eines Nationalstaates, der innerhalb gewisser Grenzen selbst definieren kann, was für inn kulturelles Eigentum darstellt (vgl. UNESCO 1970). Letztere Annahme spiegelt sich in der Aussage der UNESCO, ,[...] that cultural property constitutes one of the basic elements of civilization and national culture, and that its true value can be appreciated only in relation to the fullest possible information regarding its origin, history and traditional setting" (UNESCO 1970a:1). Kulturelles Eigentum wird durch diese Definition konstituiert als ein elementarer wertbehafteter Bestand einer "Zivilisation" und "nationalen Kultur", im Sinne Eriksens als eine "archipelago idea of culture" (Eriksen 2001:132), in dessen Folge sich die Welt in „source nations and market nations" aufteilen lässt (Merryman 1986:832) ${ }^{37}$, da, wie wir später noch lesen werden, kulturelles Eigentum und kulturelles Erbe durchaus als ökonomische Ressourcen erschlossen bzw. konzeptionalisiert werden.

Der Jurist Merryman unterteilt die UNESCO-Konzeption von kulturellem Eigentum implizit in zwei unterschiedliche Perioden. Während in beiden Perioden kulturelles Eigentum als eine Ansammlung von Objekten mit künstlerischem, archäologischem, ethnologischem und historischem Interesse gedacht wird, unterscheiden sich beide Perioden in der Subjektzuordnung eines kulturellen Eigentums in den oben dargestellten Dimensionen, d.h. zum einen von Bedeutung für die ganze Menschheit und zum anderen von Bedeutung für einen Nationalstaat (Merryman 1986:833). Der Autor schlussfolgert daraus: "These different emphases - one cosmopolitan, the other nationalist; one protective, the other retentive - characterize two ways of thinking about cultural property" (Merryman 1986:846; ich möchte hierbei betonen, dass die UNESCO zwischen 1954 und 1970 einen Politikwechsel vollzog, deren Gründe unter anderem die Entkolonialisierungsprozesse waren, in deren Folge die ehemaligen Kolonialstaaten sich emanzipierten, dementsprechende kulturpolitische Revisionen einforderten, und die UNESCO ihr statisches Verständnis von Kultur in ein dynamisch-prozesshaftes Verständnis wandelte, s. auch Kapitel 4).

Das kulturelle Eigentumskonzept wurde im internationalen Recht und auf internationaler Ebene „traditionell“ gebraucht, um den Gegenstand des „Monuments“ bzw. des „Kulturguts“ zu umschreiben und zu schützen. Beispielhaft dafür sind die 1954

\footnotetext{
${ }^{37}$ Prott\&O'Keefe fügen dieser Zweiteilung als eine dritte Kategorie die „transit countries“ hinzu (1983; vgl. Merryman 1986:832).
} 
"Hague Convention on the Protection of Cultural Property in the Event of Armed Conflict" und die 1970 UNESCO "Convention on the Means of Prohibiting and Preventing the Illicit Import, Export and Transfer of Ownership of Cultural Property". Die betreffenden Gegenstände wurden demnach primär mit dem Schutz des Eigentums assoziiert, statt den Wert und die Symbolhaftigkeit und somit die Bedeutung, die diese Artefakte für eine Gruppe von Menschen oder Gesellschaften haben, hervorzuheben. „Kulturgut“" wurde primär als handelbares Gut angesehen, das auf den Kunstmärkten der Welt zirkuliert (Blake 2000:65).

In der 1968 "Recommendation concerning the Preservation of Cultural Property Endangered by Public or Private Works" wird kulturelles Eigentum definiert als:

"(a) Immovables, such as archaeological and historic or scientific sites, structures or other features of historic, scientific, artistic or architectural value, whether religious or secular, including groups of traditional structures, historic quarters in urban or rural builtup areas and the ethnological structures of previous cultures still extant in valid form. It applies to such immovables constituting ruins existing above the earth as well as to archaeological or historic remains found within the earth. The term cultural property also includes the setting of such property;

(b) Movable property of cultural importance including that existing in or recovered from immovable property and that concealed in the earth, which may be found in archaeological or historical sites or elsewhere.

2. The term 'cultural property' includes not only the established and scheduled architectural, archaeological and historic sites and structures, but also the unscheduled or unclassified vestiges of the past as well as artistically or historically important recent sites and structures, ${ }^{38}$.

Die Anfänge der Konzeption von kulturellem Eigentum galten vor allem den Immobilien; zu schützen und zu erhalten war, was als monumental und aus „Stein und Ziegel" (Strasser 2005:54) ist. In der obigen Empfehlung bezog man erstmals auch Mobilien mit ein (vgl. ebd. 2005:54). Im Gegensatz zu internationalen Konventionen sind Empfehlungen wie die oben ausgeführte aber völkerrechtlich nicht bindend für die Vertragsstaaten (ebd.).

Die erste völkerrechtlich bindende Konvention, die ein breiteres Konzept von kulturellem Eigentum bezüglich Mobilien vorlegte, war die UNESCO „Convention on the

\footnotetext{
${ }^{38}$ Ich habe diese Definition bevorzugt, da sie die Immobilien und Mobilien besonders hervorhebt.
} 
Means of Prohibiting and Preventing the Illicit Import, Export and Transfer of Ownership of Cultural Property"von 1970 (Strasser 2005:54).

Ingesamt bezieht sich das Konzept von kulturellem Eigentum im Hinblick auf internationales Recht in seinem Kern hauptsächlich auf „Kunst“ bzw. „Kulturgut“, fassbare (tangible) Objekte bzw. bewegliche (movable, Mobilien) und unbewegliche (immovable, Immobilien) „Sachen“ aber auch Marktaustausch im weitesten Sinne. Der Begriff des „Property" bezieht sich dabei auf Begriffe, wie „Objekt“, „Monument“ oder „Mobilie“, während das Konzept „Culture“ sich auf qualitative Kriterien wie „historisch“, „archäologisch“, „ethnologisch“, „ethnographisch“ oder auch „künstlerisch“ und „kulturell“ bezieht (Frigo 2004:5).

Im weiteren Zusammenhang muss gefragt werden, wozu die UNESCO-Konventionen explizit und implizit im Bezug auf ein kulturelles Eigentum hauptsächlich dienen können? Während die Haager-Konvention zum Ziel hat, das kulturelle Eigentum mit einer Bedeutung für die gesamte Menschheit vor Zerstörung durch Krieg zu schützen und zu bewahren, so beinhalten die nachfolgenden Konventionen meist die Bestrebungen, kulturelles Eigentum vor Diebstahl, illegaler Ausfuhr und unsachgemäßer Behandlung zu schützen und internationale Regeln zu schaffen, um einen internationalen Austausch zu ermöglichen und zu erleichtern (vgl. Merryman 2005:28).

Ein weiteres kulturpolitisches Ziel der UNESCO gilt dabei unter anderem dem Erhalt und Schutz von kulturellem Eigentum, das ,[...] constitute a major contribution to the social and economic development of countries and regions which possess such treasures of mankind by means of promoting national and international tourism" (UNESCO 1968:1). Das kulturelles Eigentum als ökonomische Ressource eines territorialen Staates konzeptionalisiert wird, bekundet auch implizit die Aussage: „[...] to promote social and economic development" (UNESCO 1968:1.).

Ein zusätzliches Anliegen der meisten Konzeptionen ist, dass kulturelles Eigentum "[is] the product and witness of the different traditions and of the spiritual achievements of the past and thus is an essential element in the personality of the peoples of the world" (UNESCO 1968:1).

Es wird deutlich, dass das kulturpolitische Anliegen der UNESCO nicht nur den Schutz kulturellen Eigentums vor Zerstörung oder unsachgemäßer und illegaler Verbreitung beabsichtigt, sondern auch eine ökonomische Verwertbarkeit von kulturellem Eigentum umfasst, mit dem Ziel der Stärkung, und Entwicklung der Persönlichkeit von Menschen, sogar der gesamten Menschheit zum Ziel hat. 
Ein Grund dafür, warum die UNESCO verschiedene Typen von kulturellem Eigentum als relevant für die gesamte Menschheit erachtet, liegt nach Ansicht Kastens in der impliziten Annahme einer gemeinsamen Verantwortung "to save it from extinction" (ebd.). Als Beispiel wäre die Bewahrung der Ansprüche indigener Gesellschaften auf heiliges und/ oder geweihtes Land anzumerken, das für die Identitätsbildung dieser betreffenden Gruppen ausschlaggebend ist (vgl. Kasten 2004:21; vgl. UNESCO 2005f).

Während in den oben genannten kulturwissenschaftlich-ethnologischen Definitionen explizit keine ökonomische Relevanz des kulturellen Eigentums hervorgehoben wird (abgesehen von der Feststellung Andersons und Hanns, dass Eigentum als Knotenpunkt zwischen Recht und Ökonomie angesiedelt ist; Hann 1998a:46; Anderson 1979:40), ist dies genau der Aspekt, den die UNESCO mehrfach betont mit dem humanistischen Anliegen, dass kulturelles Eigentum ,[...] become a part of the spirit of peoples who thereby may gain consciousness of their own dignity" (ebd.).

Wie bereits weiter oben dargestellt beruhen die Konzepte der UNESCO immer auf einem implizit vorausgesetzten Entwicklungsbegriff (Albert 2002:31), der sich mit den Jahrzehnten weiterentwickelte und zum gegenwärtigen Zeitpunkt auf der kulturpolitischen Aussage der UNESCO beruht, dass kulturelles Eigentum subsumiert unter das Konzept eines kulturellen Erbes sinngemäß als funktional für die wirtschaftliche Entwicklung und als die soziale Grundlage eben dieser Entwicklung gelte. Entwicklung und Wirtschaft sind gleichermaßen Teil der Kultur eines Volkes (Schöfthaler \&Dyroff1997:21ff; vgl. Albert 2002:33f; vgl. Pérez de Cuéllar 1998:7ff; s. Kapitel 4).

Die Eigentums-Konzepte der UNESCO decken aber nicht die ganze Bandbreite kultureller Elemente $\mathrm{ab}$, die geschützt und bewahrt werden sollten. Insbesondere werden nicht diejenigen kulturellen Elemente und „Traditionen“ von Gesellschaften geschützt, die rein geistige und/ oder spirituelle Errungenschaften eines kulturellen Kontextes sind; z.B. Sprache, Tänze, Mythen, religiöse Vorstellungen, Landschaften (Prott 1992:307). Eine Erweiterung der UNESCO-Konventionen zu kulturellem Eigentum um Intellectual Property bzw. Intangible Property (geistiges Eigentum) wäre möglich gewesen. Die UNESCO entschied sich jedoch aus diversen Gründen (s. Kapitel 6), das Konzept des kulturellen Erbes in den Mittelpunkt ihrer weiteren Arbeit zu stellen und darauf aufbauend Elemente eines geistigen Erbes (Intangible/ Intellectual Heritage) auszuformulieren (vgl. Prott\&O'Keefe 1992; vgl. Blake 2000; s. Kapitel 6\&7). 
Welsh schlägt daher vor, den Begriff eines kulturellen Eigentums ganz fallen zu lassen, da der Begriff "implies a concern with origins, titles, and lines of demarcation that not be appropriate when applied to the intangibles of heritage" (1997:16). Stattdessen sollte der erstmals durch Weiner (1992) definierte Begriff „inalienable possession" verwendet werden. Dieser impliziert nach Ansicht Welsh vordergründig die konstruierte Qualität der Bedeutung einer Tatsache und deren Verknüpfung zu einem „social well-being“. Welsh argumentiert, dass: „Understanding the reasons for attachment to possession, [...], has less to do with understanding the source of rights than with understanding the consequences of loss" (ebd.; vgl. Brown 2004:53). Brown stimmt dem Ansinnen Welsh zu, weil seiner Ansicht nach die Verwendung des Begriffs "inalienable possession [...] shifts the focus from economic questions to matters of community survival and human dignity" (2004:53). Die permanente Verwendung des Begriffs eines kulturellen Eigentums würde zudem "extend the influence of Euroamerican values in the guise of supporting a return to traditionalism" (Welsh 1997:13; vgl. Brown 2004:53).

Die neueren Konventionen der UNESCO zielen zwar im Kontext nicht mehr ausdrücklich auf kulturelles Eigentum, aber auf kulturelles Erbe ab. Die UNESCO versucht durch ihre rezente Kulturpolitik, die Entscheidungsgewalt über kulturelles Eigentum jenen lokalen Gruppen zuzusprechen, die auch bisher die Kontrolle über dieses vermeintliche Eigentum im Sinne der UNESCO hatten (Kasten 2004:21; vgl. UNESCO 2005f). Die Vorgaben der UNESCO gelten mittlerweile insbesondere dann, wenn nationale oder auch internationale Institutionen versuchen, auf Ressourcen lokaler Gruppen, bzw. indigenen Gesellschaften zuzugreifen (Kasten 2004:21). Dieser kulturpolitische Richtungswechsel ist meiner Ansicht nach in direktem Zusammenhang mit dem oben beschriebenen policy-turn zu sehen.

Kulturelles Eigentum kann in einer Vielzahl von Möglichkeiten instrumentalisiert werden. Heutzutage wird kulturelles Eigentum oftmals auch als Legitimation indigener Ansprüche auf Territorien oder anderer Ressourcen gefördert oder sogar erfunden (Kasten 2004:9). Auf internationaler Ebene bemüht sich gerade die UNESCO mit ihrer neuesten Konvention zur kulturellen Vielfalt, diesen Ansprüchen gerecht zu werden und unter anderem den multiplen Formen von Eigentumskonzeptionen, ob geistiger oder materieller Art, auf lokaler und regionaler Ebene internationale und nationale Rahmenbedingungen zu schaffen (vgl. Kasten 2004; vgl. Kasten 2002; vgl. UNESCO 2005f). Internationale Regelungen sind nach Ansicht Merrymans unerläss- 
lich, wenn kulturelles Eigentum erhalten und geschützt werden soll, damit sich die "gesamte Menschheit" auch weiterhin daran erfreuen kann und diese Art von Eigentum in sicheren Bahnen global zirkuliert. Dies hat unter anderem den Vorteil für indigene Gruppen, ,„[a]s to „poor nations“, many of which are source nations, the orderly marketing of surplus cultural object could pro tanto displace the black market, while providing a significant source of income to the source nation and its citizens"' (Merryman 2005:23).

Die "commodification of culture" und deren politische Instrumentalisierung für die Interessen und Ansprüche indigener Gruppen sind derzeit Schlüsselthemen einer rezent in vielen wissenschaftlichen Disziplinen geführten Debatte über kulturelles Eigentum (Kasten 2004:13). Die Fragen nach Authentizität und inwieweit kulturelles Eigentum als Teil eines kulturellen Erbes in Form von Individualeigentum, Kollektiveigentum oder als ein Eigentum der gesamten Menschheit unterscheiden und interpretiert werden kann und wird, ist für den Rahmen dieser Arbeit, um es mit Theodor Fontane zu sagen, „[...] ein zu weites Feld“ (Fontane 1963:296; Hervorhebung im Original). 
„Ich kann freilich nicht sagen ob es besser werden wird, wenn es anders wird; aber so viel kann ich sagen, es muss anders werden, wenn es gut werden soll“"

(Lichtenberg 1994:111)

\section{Von kulturellem Eigentum zu kulturellem Erbe}

Das Konzept eines kulturellen Erbes wurde international erstmals mit der Welterbekonvention 1972 ausformuliert (vgl. Frigo 2004; vgl. Prott 1992; vgl. Blake 2000). Der Begriff des kulturellen Erbes wird jedoch bereits seit der 1954 Haag-Konvention verwendet. In der Präambel des Textes steht geschrieben:

"Considering that the preservation of the cultural heritage is of great importance for all peoples of the world and that it is important that this heritage should receive international protection" (UNESCO 1954).

Der übrige Text der Konvention bezieht sich ausschließlich auf kulturelles Eigentum. In Artikel I der Konvention wird kulturelles Eigentum definiert ohne Bezug darauf zu nehmen, was dem gegenüber kulturelles Erbe sei und welchen Stellenwert kulturelles Eigentum gegenüber kulturellem Erbe hat bzw. in welchem Zusammenhang beide Konzepte stehen. Interessanterweise werden die beiden Konzepte nie simultan oder gleichwertig in einer Konvention, Empfehlung oder Erklärung genutzt und definiert (Frigo 2004:5). Der gleiche Fall, nur im umgekehrten Verhältnis, kommt in der „Draft Convention on the Protection of the Underwater Heritage and Explanatory Report" (1985) vor. Die Konvention wurde durch ein ad hoc Expertenkomitee erstellt. In Artikel I, Paragraph I wird festgestellt:

„For the purposes of this Convention all remains and objects and any other traces of human existence [...] shall be considered as being part of the underwater cultural heritage, and are hereinafter referred to as underwater cultural property" (vgl. Blake 1996: 819; vgl. Frigo 2004: 5; ICOMOS 1985).

In der Welterbekonvention wird ebenso, wie in verschiedenen anderen internationalen Rechtstexten, die den Schutz und das Bewahren von kulturellem Eigentum oder kulturellem Erbe als Kernkonzept verfolgen, keine eindeutige Abgrenzung zwischen diesen beiden Konzepten gemacht. Prott und O'Keefe bemerken zur Welterbekonvention: 
"The Preamble emphasizes the change by referring to 'the existing international conventions, recommendations and resolutions concerning cultural and natural property' whereas every else in the text one finds the phrase 'cultural heritage" (Prott\&O'Keefe 1992:318).

Um es mit Durkheim zu sagen:

„Diese Gedankenbildungen oder Begriffe oder wie man sie auch nennen will, sind nicht die legitimen Vertreter der Dinge. [...] Sie sind von der Praxis und für die Praxis geschaffen. Ein Gedanke kann nun praktisch ganz wohl eine nützliche Rolle spielen und theoretisch falsch sein“ (1908:39f).

Tatsache ist, dass die verwendeten Begriffe keineswegs ausschließlich wissenschaftlich konzipiert sind. Die Erstellung internationaler Rechtsinstrumente, wie völkerrechtlich bindende Abkommen, entspringt im Grunde dem politischen Milieu. Die jeweilig zuständigen Institutionen sollten - laut Frigo - bei der Verwendung der Begrifflichkeit aber darauf achten, dass jeder terminus technicus für sich ein definiertes Konzept darstellt und beide Konzepte innerhalb eines rechtsverbindlichen Textes aufeinander abgestimmt sind (ebd. 2004: 5f). Bereits 1908 beschreibt Emil Durkheim - zwar für das methodische Vorgehen der Soziologie -, dass man nicht mit Altagsbegriffen bzw. den "notiones vulgares“ operiere, sondern diese vorerst untersuchen und daraufhin inhaltlich ausformen soll, um die zu beschreibende Sache objektivierbar zu machen.

$\mathrm{Da}$,wie oben bereits dargestellt, bei den Begriffsbestimmungen und Übersetzungen Schwierigkeiten entstehen, kommt Janet Blake, die eine Vergleichsstudie über die bestehenden Vorschriften anfertigte, zu dem Schluss, diese Situation als „Minenfeld“ zu bezeichnen (Blake 2002:7; vgl. Strasser 2005:67). Die meisten Begriffe, die bei der Umschreibung dieser Konzepte immer wieder Verwendung finden, wie z.B. „traditional“, „popular“, „living“, „oral“, „folklore“, „,heritage“, „property“, „cultural" sowie „intellectual“ oder auch „naturaf”, (die Verhandlungen über Konventionen und Empfehlungen werden zumeist in Englisch oder Französisch geführt; vgl. Strasser 2005:70), besitzen in den verschiedensten Regionen der Welt unterschiedliche Bedeutung (vgl. Blake 2002:7f, s.o.). 
Gerade bei Themen wie kulturellem Eigentum und kulturellem Erbe wäre es - meiner Ansicht nach - unerlässlich, die verwendeten Begriffe, besonders in internationalen Konventionen und Empfehlungen vorab objektivierbar zu machen bzw. einer intersubjektiven Überprüfung zu unterziehen, um sie daran anschließend zu definieren (vgl. Durkheim 1908:38ff). Frigo (2004:6) bemerkt dazu:

"To provide legal protection for the cultural property concerned, it may be of great importance to ascertain the link with a specific community. This would require clarifying to which cultural heritage the property is assumed to belong" (vgl. Merryman 1986:831; ebd. 1994:61).

Auch die Feststellung G. P. Murdocks stellt treffend klar, dass:

"A really adequate study of property rights and inheritance in cross-cultural perspective still remain to be made" (Murdock 1949, S. 39).

Auf internationaler Ebene hat sich bei der Ausarbeitung der Konzeptionen in Bezug auf kulturelles Eigentum und kulturelles Erbe zwischen der Haag-Konvention von 1954 bis heute viel verändert. Wie oben erwähnt, kann das kulturelle EigentumsKonzept aufgrund einiger konzeptioneller Mängel nicht alle Facetten eines schützenswerten kulturellen Kontextes, im Sinne der UNESCO, erfassen. Mit der Welterbekonvention von 1972 bzw. der „Convention concerning the Protection of the World Cultural and Natural Heritage“"wurde erstmalig kulturelles Erbe hervorgehoben.

Wie kam es dazu? Welche Vorteile liegen im Konzept eines kulturellen Erbes oder welche Nachteile liegen im Konzept eines kulturellen Eigentums?

\subsection{Eigentum als euro-amerikanisches Konzept}

Der Gegenstandsbereich, der durch das „Cultural Property“ Konzept der UNESCO geschützt wird, ist primär als „Eigentum“ definiert, das, wenn möglich, als handelbares Gut auf Märkten gekauft und verkauft werden kann. Kulturelles Eigentum wird im internationalen Recht als ein eigenständiger Bereich im Eigentumsrecht verstanden, wie z.B. auch „personal property and intellectual property“ (Prott\&O’Keefe 1992:312). Normative Orientierung und Symbolhaftigkeit jener Objekte stehen demnach nicht im Vordergrund (vgl. Blake 2000:65). Prott \& O'Keefe bemerken: 
„It is an especially Western concept and has particular commercial connotations: it implies control by the owner expressed by his ability to alienate, to exploit and to exclude others from the object or site in question" (Prott\&O'Keefe 1992:310).

„Property“, übersetzt als Eigentum, ist eine der fundamentalsten Konzeptionen, um die herum sich eine Vielzahl an politischen, philosophischen und anderen Theorien entwickelt haben. Das Konzept birgt eine große Spannbreite an ideologischen Implikationen mit der Schwierigkeit, diese außer Acht zu lassen zu können, wenn man das Konzept in Bezug auf ein kulturelles Eigentum bzw. kulturelles Erbe verwendet. Besonders in Fällen, in denen man versucht, die herkömmlichen Assoziationen und Werte, die dem Begriff des Eigentums innewohnen, wegzulassen, um ein gewünschtes gesellschaftliches Ziel zu erreichen; z.B. Landnutzung, Umweltschutz oder eben den Schutz von kulturellem Erbe (vgl. Prott\&O'Keefe 1992:309; vgl. Blake 2000:65). Blake argumentiert: "It is problematic to apply a legal concept involving the rights of the possessor to the protection of cultural resources which may involve a severe curtailment of such rights and the separation of access and control from ownership" (ebd. 65).

\subsection{Mullick versus Mullick: Ein Fall über materielles bewegliches Eigentum $^{39}$}

Im Frühling des Jahres 1925 wurde in der Zeitschrift „The Times“ein für die damalige Zeit außergewöhnlicher Fall am High Court of Calcutta diskutiert. Mutty Lal Mullick, ein Angehöriger des Hinduismus aus Kalkutta, versah seinen Haushalt mit verschiedenen göttlichen Idolen ${ }^{40}$. Nach seinem Tod ging der Schutz der Idole auf seine Witwe über. Diese wiederum gab die Idole an ihren Adoptivsohn Jadulal und dessen Sohn weiter. Die Frage, die bei diesem Fall behandelt wurde war, ob Jadulal nach Weitergabe des Idols an inn nun auch der „Vormund“ bzw. Nutznießer des Idols wurde und den "Gegenstand" in sein eigenes Haus bringen dürfte. Nach hinduisti-

\footnotetext{
${ }^{39}$ Ich übersetze tangible movable property als materielles bewegliches Eigentum.

${ }^{40}$ Idol leitet sich vom griechischen eidolon ab, und bedeutet „Gestalt, Bild“. In der Ethnologie bezeichnet man mit diesem Begriff Objekte der Verehrung, meist plastisch und von anthropo- oder zoomorpher Gestalt. (Schoenfelder 1999:183; vgl. Duff 1929:42; ich gehe hier nicht weiter auf die genaue Bezeichnung und Bedeutung der Idole Thakurani Sri Sri Singabahini Debi und Salgram Sila ein).
} 
schem Glauben und Recht ist ein so genanntes „Idol" aber zugleich eine Rechtsperson, die klagen und angeklagt werden kann. Die Interessen des Idols werden durch die Person vertreten, die dieses Idol verehrt bzw. sein "Manager“ ist oder durch einen „nahen Freund“ (vgl. Prott\&O’Keefe 1992:310; Duff 1929:42). Das Gericht wurde mit einem Fall betraut, in dem ein - nach dem Verständnis im euro-amerikanischem Recht - "Gegenstand“ (tangible movable property) eine öffentliche Rechtsperson mit eigenen Rechten und Pflichten darstellt. Es musste geklärt werden, ob das Begehren Jadulal's mit dem Begehren des Idols vereinbar war. Das Idol war eben nicht eine bloße Habe, die man besitzen und handeln kann, wie es bei weltlichem Eigentum (secular property) möglich ist (vgl. Duff 1929: 46f, Prott\&O'Keefe 1992:310).

Diese Art des Beispiels zeigt, dass, wenn das Konzept des kulturellen Eigentums angewandt wird, es mit großer Sorgfalt genutzt und in manchen Fallen neu interpretiert werden muss. Das Potenzial eines Missverständnisses ist besonders hoch, daher sollte, wenn möglich, der Begriff bzw. das Konzept des Erbes und der kulturellen Vielfalt angewendet werden, um die ideologische und historische Belastung des Eigentums-Konzepts auszuschalten (Prott\&O'Keefe 1992:310).

\subsection{Eigentum als ökonomischer Begriff}

Gegenüber des mit einer großen Spannbreite von Emotionen und Werten behafteten Begriffes „Eigentum “ - von John Locke bis zum Kommunismus und darüber hinaus -, ist „kulturelles Erbe" eine relativ neue Begriffskonstellation mit wenig ideologischem Ballast (Prott\&O'Keefe 1992:309).

Der Verwendung des terminus technicus „kulturelles Eigentum“ ist im euroamerikanischen Kontext die Idee der Übertragbarkeit inhärent. Diese Möglichkeit einer Übertragung kann den Marktwert des Objektes bestimmen, d.h. kulturelle Artefakte und ähnlicher Objekte unterliegen einer "commodification“ und werden als Waren verkauft und gekauft. Mitbestimmend dabei ist das klassische Prinzip von Angebot und Nachfrage (vgl. ebd.). Prott\&O'Keefe konstatieren: "Yet this way of delineating an individual or group's relationship to a thing may be quit alien in other societies" (ebd. 1992:310). 


\subsection{Kulturelles Eigentum und die Kategorie des Immobilen/ Geisti- gen}

Eines der gewichtigsten Argumente gegen eine Verwendung des kulturellen Eigentums-Konzepts ist - wie oben erwähnt - die Beschränktheit der Konzeption, um alle kulturellen Elemente sinngerecht bzw. nicht als Ware abzudecken, u.a. Monumente, archäologische Fundstätten, Felsmalereien, Ethnographika, Heilige Orte und Ritualplätze, Landschaftselemente mit einer kulturellen Signifikanz für Menschen, Kleidung, Waffen, tägliche Gebrauchsgegenstände, rituelle Gebrauchsgegenstände, Musikinstrumente, verschiedenste Objekte mit einem historischen Charakter; z.B. Obsidian, Fossilien, Pollen und viele Elemente mehr. Neben den oben beschrieben physisch greifbaren Dingen (tangible) gibt es aber auch geistige, immaterielle (intangible) Elemente, die schützens- und erhaltenswert sind; z.B. Orale Traditionen, Wissen über den Bau von Schiffen oder Schmelzöfen, Lieder, zeremonielle und rituelle Weisheiten, der „way of life" einer Gesellschaft, Sprachen oder auch die Beziehung von Menschen zu ihrem Land, das sie bewohnen. Die letzteren Beispiele geistiger/ immaterieller Elemente machen die extreme Begrenztheit des kulturellen Eigentums-Konzepts besonders deutlich, wenn man die kulturellen Elemente betrachtet, die ausschließlich durch die Erhaltung von Vorstellungen, Glauben und oraler Traditionen vermittelbar sind (vgl. Blake 2000:66). Prott (1998:222) bemerkt zum Konzept eines kulturellen Eigentums: "that it is a purely Western legal category which is far too narrow and that it has been global influences that have allowed the broadening of the concept of cultural heritage" und das: "property' has some kind of fundamental importance in our culture [...]" (Prott\&O'Keefe 1992:309 - Hervorhebung durch mich).

Historiker, Soziologen und speziell Archäologen und Ethnologen weisen darauf hin, dass es essentiell sei, Informationen unterschiedlichster Art zu bewahren, nicht nur eine Kategorie des „Fassbaren“ (tangibles), mit "Immobilien“ (immovables) und „Mobilien“ (movables), sondern gerade die Kategorie des "Nicht-fassbaren, Geistigen, Spirituellen, Intellektuellen" (intangibles) sei wichtig. Zum Beispiel ist es für die euroamerikanische Wissenschaft von großer Evidenz zu wissen, wie ein Musikinstrument gespielt wird, bei welchen Anlässen und von wem. Denn eben diese Elemente tragen zu einem großen Teil zu unserem besseren Verständnis der Umstände bei, in denen sich das etwaige Element bewegt bzw. aus denen es kommt. Ein kulturelles Element, 
wie etwa ein Musikinstrument, dessen orale und textuale Information verloren ging, ist - laut Prott\&O'Keefe (1992:308) - für den kulturellen Kontext, aus dem es stammt, weniger von Nutzen, als wenn die Informationen darüber erhalten wären.

Kulturelles Eigentum umfasst weitestgehend die meisten Kategorien, aber eben nicht alle. Es beinhaltet die ersten zwei Kategorien des „Fassbaren“, d.h. die Immobilien (z.B. Monumente) und die Mobilien (z.B. Grabbeigaben, Gebeine), aber bereits bei der dritten Kategorie des „Nicht-fassbaren“ erweist sich das Konzept als mangelhaft. Rituale, Zeremonien, orale Traditionen und darstellende Künste werden nicht mehr berührt. Das Konzept versagt insbesondere dann, wenn es um den Schutz und die Bewahrung von textualen und oralen Informationen bezüglich der Gegenstände innerhalb der anderen Kategorien geht (vgl. Prott\&O’Keefe 1992:313).

\subsection{Die fundamentale Politik hinter kulturellem Eigentum und Erbe}

Die Mitgliedstaaten der UNESCO sind sich weitestgehend darüber einig, dass nicht alles bewahrt werden kann und sollte. Auf der anderen Seite besteht ein kulturelles Erbe nicht nur aus ein paar vereinzelten Objekten oder anderen Elementen, die durch eine nationale oder internationale Legislative dafür ausgewählt wurden (Prott\&O'Keefe 1992:309).

Die fundamentale Politik hinter kulturellem Eigentum ist, wie oben bereits angemerkt, der Rechtsschutz des Eigentümers, definiert als das Recht auf Verwertung, Übertragung und Exklusion, wie Prott\&O'Keefe anmerken: "If this policy is carried to its logical conclusion then the owner can buried with a painting that he purchased for millions of dollars but which represents a peak achievement of human culture" (1992:309).

Die fundamentale Politik hinter kulturellem Erbe hingegen ist der Schutz des "Erbes", damit gegenwärtige und zukünftige Generationen sich daran erfreuen können. Dies bedeutet nicht ausschließlich physischen Schutz, sondern auch die Möglichkeiten des Zugangs zum Gegenstand durch andere Personen als die des Eigentümers. Diese Konzeption beinhaltet also Einschränkungen der Rechte des Eigentümers, gleich, ob es eine Einzelperson, eine öffentliche Person, eine Gemeinschaft oder ein Staat ist (ebd. 310). 
Die genaue Beziehung zwischen kulturellem Eigentum und kulturellem Erbe bleibt jedoch unklar. In manchen Fällen sind die Konzepte austauschbar, während in anderen Fällen kulturelles Eigentum als eine Sub-Kategorie innerhalb des kulturellen Erbes verstanden wird. In der Preamble der "Recommendation concerning the Preservation of Cultural Property Endangered by Public or Private Works" (UNESCO 1968) ist hierzu ein sinngemäßer Passus:

"Considering that cultural property is the product and witness of the different traditions and of the spiritual achievements of the past and thus is an essential element in the personality of the peoples of the world [...]".

Die Bedeutung von kulturellem Eigentum geht demnach weit über die von Objekten hinaus und gliedert sich in ein umfassenderes Ganzes ein. Kulturelles Erbe ist die gegenwärtig wesentliche Konzeption geworden, da sie fähig dazu ist, eine größere Spannbreite von möglichen Elementen abzudecken, einschließlich kulturellem Eigentum und des Immateriellen (Blake 2000:67).

Was genau ist dann kulturelles Erbe? Wie ist es definiert und wie stellt es sich in internationalen Konventionen dar? Welche Probleme existieren? Oder ist alles wie es sein soll? 
"Ebenso wie man einen Keim, damit er Kristalle bilde, in eine gesättigte Lösung einführen muß, ist es notwendig, dieser Gesamtheit uns fremder Zeugnisse so etwas wie den Samen eines Erinnerns zuzuführen, damit sie sich zu einer konstanten Masse von Erinnerungen festigt"

(Halbwachs 1991:5)

\section{Kulturelles Erbe}

Außergewöhnliche Stätten der Welt zu katalogisieren, zu schützen und zu bewahren ist keine neue Idee (s. Kapitel 2). Der rapide Fortschritt der Gefährdung und Vernichtung des natürlichen und kulturellen Erbes der Erde - laut Mayerhofer - seit dem Beginn der Industrialisierung ließ zunächst in den betroffenen Industrienationen das Bewusstsein wachsen, dass massive Bestrebungen nötig seien, um die kulturellen Manifestationen nationaler Identität zu bewahren. In der Folge reifte die Überzeugung, dass nationaler Schutz nicht immer ausreichend geboten werden kann. Eine Überzeugung, die durch die Verwüstungen und Zerstörungen des zweiten Weltkriegs bestärkt wurde und sich schließlich u.a. in der Verabschiedung diverser Konventionen und Abkommen innerhalb und außerhalb der UNESCO, auf nationaler, europäischer und internationaler Ebene niederschlug (s.o.; Mayerhofer 1996:54).

\subsection{Die Anfänge der Idee eines internationalen Kultur- und Naturer- bes}

Zu Beginn der 1960'er Jahre griffen die Mitgliedstaaten der UNESCO die Idee eines Fonds zum Schutz des kulturellen Erbes auf. Erste Überlegungen gingen bereits auf die 1940'er Jahre zurück, als international diskutiert wurde, ob es sinnvoll wäre, eine "Touristensteuer" bei Einreise in ein Land zu erheben, um damit einen internationalen Fond zu speisen. (Strasser 2005:58). Bis zu diesem Zeitpunkt befasste sich der Schutzgedanke in der internationalen Zusammenarbeit hauptsächlich mit Kulturgütern (s.o.).

Immer lauter wurden jedoch auch jene Stimmen, welche sich für den gemeinsamen Schutz von Kultur und Natur einsetzten. Eine wichtige Rolle spielte in diesem Zusammenhang der US-Amerikanische Umweltaktivist und Gründungsmitglied des 
World Wildlife Funds (WWF) Russel E. Train. Bereits 1965 forderte er als Mitglied des Komitees für Natürliche Ressourcen an einer Konferenz im Weißen Haus eine Welterbe-Stiftung (World Heritage Trust) zur Förderung der internationalen Zusammenarbeit. Diese Kooperation war hauptsächlich zur Identifizierung und Bewahrung der, wie es damals hieß: „world's superb natural and scenic areas and historic sites for the present and the future benefit of the entire world citizenery" gedacht. 1967 schilderte Russel Train sein Konzept am International Congress on Nature and Man in Amsterdam (Train 2002: 1f; vgl. Bolliger et al 2004:16f).

\subsubsection{Die Rolle der U.S.A.}

Zu Beginn der 1970er Jahre unter Präsident Nixon wurden globale Umweltanliegen in den USA vermehrt thematisiert. In der Zeitschrift „Environmental Message“, welche die Handschrift Russel Trains (Vorsitzender des neugegründeten Council of Environmental Quality) trug, äußerte Nixon sein Anliegen, die weltweite Kooperation in Umweltfragen anzukurbeln. Seine Idee war es, das hundertste Jubiläum des Yellowstone National Parks mit einer neuen Konvention symbolisch zu untermauern (Train 2002: 2; vgl. Bolliger et al 2004:17f).

Jedoch nicht nur die USA befasste sich mit dem Gedanken einer neuen Konvention. Sowohl die Internationale Naturschutzunion (IUCN), der Internationale Rat für Denkmalpflege (ICOMOS), wie auch die UNESCO unter der Leitung von Michel Batisse (UNESCO-Direktor für die Abteilung Wissenschaft und Organisator der Biosphere Conference 1986) setzten sich intensiv mit dem Gedanken einer gemeinsamen Konvention für Kultur- und Naturerbe auseinander. Die verschiedenen Expertengruppen kamen an der von den Vereinten Nationen organisierten Konferenz zur menschlichen Umwelt im Juni 1972 in Stockholm zusammen und debattierten über die Idee einer Welterbekonvention (Bolliger et al 2004:17f). 


\subsubsection{Die Welterbekonvention von 1972}

Im Jahre 1972 manifestierte sich in dem Übereinkommen zum Schutz des Kulturund Naturerbes der Welt (UNESCO 1972, Welterbekonvention) der Wille der Staatsgemeinschaft zu internationaler Zusammenarbeit. Anfänglich mit dem Bestreben, erstmals Kultur- und Naturgüter zusammen unter den Schutz eines globalen Menschheitserbes zu stellen. Die Welterbekonvention ist die bekannteste Konvention der UNESCO im Bereich des Kultur- und Naturerbeschutzes. Die 17. Generalkonferenz (17.10. - 21.11.1972) verabschiedete das Übereinkommen zum Schutz des Kultur- und Naturerbes, das 1975 in Kraft trat. Bis heute sind der Konvention 181 Staaten beigetreten (UNESCO 2005c; Mayerhofer 1996:54; vgl. Reuther 1996:179). Neben der Einrichtung eines Fonds wurde ebenfalls diskutiert, welche Art des kulturellen Erbes schützenswert sei. Das Konzept der Welterbekonvention basiert auf der Idee, dass die Vertragsstaaten gemeinsam für das von der Staatengemeinschaft als von „außergewöhnlichem, universellem Wert“ (outstanding universal value; UNESCO 1972: Präambel) bezeichnete Erbe der Welt zuständig sind (Mayerhofer 1996:54; vgl. Strasser 2005:58; vgl. Bolliger et al 2004).

Begründet wurde die Notwendigkeit einer finanziellen Förderung für den Schutz von Denkmälern mit weltweiter Bedeutung damit, dass: "jene Denkmäler fast ausschließlich aus weitläufigen und monumentalen Ensembles" (Strasser 2005:58.) bestehen würden. Auf Vorschlag der USA wurde die Kategorie des "Naturerbes“ mit in die Konvention integriert. Es galt der Umweltschutzbewegung der 1960'er und 1970'er Jahre zu verdanken, die die Sichtweise vertraten, dass sich Natur und Kultur gegenseitig bedingen und ergänzen (Strasser 2005:58; s.o.). Natur und Kultur wurden jedoch weitgehend stofflich gefasst. Erst auf der Weltkulturkonferenz in Mexiko im Jahre 1982 erweiterte man dieses materielle Verständnis von dem, was zu schützen gilt, um ein immaterielles Verständnis. Ebenso überarbeitete man das Kulturkonzept (s.o). Es wurde um das „einzigartige geistige, interkulturelle und emotionale Erbe der Menschheit [...] " erweitert (Fabrizio 1997:6; vgl. Albert 2002:31).

Bis heute hat die UNESCO insgesamt 812 Objekte in 137 Staaten unter den Schutz der Konvention gestellt. Davon fallen 628 auf den Sektor des kulturellen Erbes, 160 Objekte auf den Sektor des Naturerbes und 24 sind als ein „Mix“ aus Kultur- und Naturerbe anerkannt (UNESCO 2005a). 
Die Konvention funktioniert substantiell über drei Organe: einer Liste des Weltkulturund Naturerbes, einem Komitee und einem Welterbefond. Jeder Vertragsstaat zahlt ein Prozent seiner Mitgliedsbeiträge in den Fond ein. Die zur Verfügung stehenden Mittel werden dann dorthin transferiert, wo die eigenen Ressourcen der Vertragsstaaten zu gering sind. Der Sinn des Fonds besteht eher im multilateralen Handeln als darin, den restaurativen Herausforderungen standzuhalten (Bernecker 2002:23).

Das Welterbekomitee ist das Exekutivorgan der Konvention. Es entscheidet auf jährlichen Sitzungen über die Aufnahme neuer Stätte, Einschreibungen in die „Rote Liste ${ }^{\text {“41 }}$ sowie ggf. Streichungen aus der Liste. Das Komitee ist ein demokratisch gewähltes Vertretergremium, das die Unterzeichnerstaaten der Konvention mit der Erarbeitung der zu treffenden Entscheidungen beauftragt. Die alle zwei Jahre stattfindende Generalversammlung der Vertragsstaaten wählt jeweils sieben aus ihren Reihen für sechs Jahre in das aus 21 Staaten bestehende Komitee (ebd.24).

Die UNESCO kooperiert zur Wahrnehmung ihrer Aufgaben u.a. mit der ICOMOS und der IUCON. Diese politisch unabhängigen Weltfachverbände stellen die wissenschaftliche Expertise, wenn Staaten Anträge eingereicht haben. Als Richtschnur gelten die "Operational Guidelines for the Implementation of the World Heritage Convention” (UNESCO 2005d; Bernecker 2002:24).

\subsubsection{Die Generierung der Kategorie eines immateriellen Erbes}

In den dreißig Jahren des Bestehens des Abkommens entwickelte sich der Begriff des kulturellen Erbes und somit des Welterbes weiter. Blieb die Konvention in ihren Anfängen zunächst noch auf die Kategorie des materiellen Kulturerbes (tangible) begrenzt - sowohl die Immobilien, wie auch die Mobilien (bereits in den Konzeptionen eines kulturellen Eigentums waren dies die erwähnten Bestandteile, die es zu schützen und zu bewahren galt; s.o.) - so wurde im Laufe der Jahrzehnte die Konvention und das Konzept des immateriellen Kulturerbes (intangibles) erweitert (Strasser 2005:60). Bereits seit dem Ende der 1960'er Jahre begann man, Teile

\footnotetext{
${ }^{41}$ Mit der Eintragung in die "Rote Liste" will die UNESCO die Aufmerksamkeit der politisch Verantwortlichen und das öffentliche Interesse am Schutz der weltbedeutenden Kultur- und Naturerbestätten wecken, die durch menschliche Eingriffe oder Naturkatastrophen gefährdet sind. $n$ der Liste des Welterbes führt das Zwischenstaatliche Komitee der UNESCO für den Schutz des Natur- und Kulturerbes derzeit 33 Stätten als "besonders gefährdet" (UNESCO: http://www.unesco.de/c_arbeitsgebiete/welterbe_rote_liste.htm; 01.06.2006; 20 Uhr).
} 
eines immateriellen Erbes international durch das Urheberrecht zu schützen. Dieser Ansatz, der nur im Bereich des individuellen Schöpfens des Erbes bei gewerblicher Nutzung einen gewissen rechtlichen Schutzstandard in euro-amerikanischem Sinne gewährt, lässt sich nicht generell auf das immaterielle Erbe anwenden (Blake 2002:22ff; vgl. Strasser 2005:68).

Der Begriff des immateriellen Kulturerbes und das mit inm verknüpfte Potential sind weitestgehend aus einem internationalen Diskurs über eine globale kulturpolitische Neuorientierung entsprungen (vgl. Beier-de Haan 2005:58, abgesehen von der „Erfindung" eines kulturellen Erbes seit der späten Neuzeit mit Beginn der Französischen Revolution; s. Kapitel 2).

Betrachtet man die Welterbeliste näher, fällt auf, dass die „ärmeren“ Länder auf der Liste stark unterrepräsentiert sind. Munjeri (2004:16) bemerkt hierzu:

"[...] historic towns and religious buildings (cathedrals, etc.) were over-represented; the architecture was 'élitist' (castles, palaces etc.), in general terms all living cultures especially traditional ones with their depth, their wealth, their complexity and their diverse relationships figured very little on the list [...]".

Ein Grund dafür ist, dass sich auch hier die Ungleichheiten zwischen den so genannten "Ländern des Norden“ und des „Südens“ insofern auswirken, als insbesondere viele afrikanische Länder die aufwendigen Erhaltungsrichtlinien nicht erfüllen konnten und können. Ein anderes Faktum ist, laut Albert, dass innerhalb der Expertengruppen der UNESCO afrikanische, lateinamerikanische und asiatische Experten gegenüber den Euro-amerikanischen unterrepräsentiert sind. Albert hält fest, dass auch die UNESCO eine Organisation ist, die das, was es weltweit zu erhalten gilt, mit den Maßstäben der westlichen Industrieländer misst (2002:31f).

Regionen, in denen das euro-amerikanische Modell des im Abkommen festgeschriebenen Kulturerbegedankens nicht anzuwenden war, hatten somit auch weniger Möglichkeit, inr kulturelles Erbe auf die Liste zu setzen. Das Komitee gewann schließlich die Einsicht, von einer strikten Anwendung des Konzepts eines kulturellen Eigentums bzw. des materiellen Kulturerbes absehen zu müssen, da z.B. Bautraditionen in Afrika und Asien keine Aussichten auf Eintragung in die Liste hätten (viele Bauwerke werden in regelmäßigen Abständen vollständig erneuert, da sie zumeist aus Holz bestehen und somit starken Verwitterungserscheinungen ausgesetzt sind. Den Bauwerken fehlt somit das euro-amerikanische Kriterium der „Authentizität“; d.h. sie seien nicht mehr „original“). 
Eine Analyse des Problems durch eine Expertenkommission der UNESCO im Jahre 1994 ergab:

"From its inception the World Heritage List had been based on an exclusively, monumental' concept of the cultural heritage [...] the idea of cultural heritage had been embodied in and confined to architectural monuments [...] A static view of human cultures" (UNESCO 1994a:1ff).

Die entscheidende Aussage des Expertentreffens war:

„[...] that the history of art and architecture, archaeology, anthropology and ethnology was no longer concentrated on single monuments in isolation but rather on considering cultural groupings that were complex and multidimensional, which demonstrated in spatial terms the social structures, ways of life, beliefs, systems of knowledge, representations of different past and present cultures in the entire world. Each individual piece of evidence should therefore be considered not in isolation but within its whole context with an understanding of the multiple reciprocal relationship that had its physical (i.e. tangible) and nonphysical (i.e. intangible) environment" (ebd.).

Die UNESCO beschäftigt sich seit einigen Jahren mit der Frage nach dem über das materielle Erbe hinausgehende Immaterielle Kulturerbe, bzw. all das, was sich nicht materiell verfestigt hat (Beier-de Haan 2005:58; vgl. Strasser 2005: 60f). Anlässlich der Vorbereitungen der weltweiten Dekade für kulturelle Entwicklung der UN von 1988-1997 wurde bei der UNESCO der Vorschlag eingebracht, die Inventarisierung, Sammlung und Erhaltung der verschiedenen Formen des „nicht-körperlichen“ Erbes (Non-physical Heritage) einschließlich mündlicher Überlieferungen (Oral Traditions) unter den Mitgliedstaaten zu fördern (Strasser 2005:70). 1987 ging man schließlich von der Idee eines reinen Inventars ab und begann mit der Ausarbeitung eines Aktionsplanes zum Schutz und zur Darstellung des immateriellen Kulturerbes (Valderama 1995:154; vgl. Strasser 2005:70f).

Als Vorläufer der Konvention zum Schutz des Immateriellen Kulturerbes gilt die 1989 verabschiedete Empfehlung zum Schutz der traditionellen Kultur und Folklore (Recommendation on the Safeguarding of Traditional Culture and Folklore). Die Empfehlung wies jedoch Schwächen im Bereich der Begriffsbestimmungen, des Umfangs und der Schutzmechanismen auf (Blake 2002:32f).

Im Jahre 2001 hat die UNESCO das immaterielle Kulturerbe (Intangible Heritage) zum programmatischen Ziel ihrer Aufgaben und Aktivitäten gemacht, indem sie es 
neben dem materiellen Kulturerbe (Tangible heritage) zum Bestand des kulturellen Erbes (Cultural Heritage) erklärt hat (Strasser 2005:70f).

Innerhalb von drei Jahren der Verhandlung auf Expertenebene konnte im Jahre 2003 schließlich ein eigenes Abkommen zum Schutz des Immateriellen Kulturerbes verabschiedet werden (UNESCO 2003a).

Haben sich die Vorstellungen von kulturellem Erbe lange Zeit auf bauliche Überreste und materialisierte Elemente eines kulturellen Kontextes beschränkt, so ist das Verständnis von kulturellem Erbe mittlerweile um wesentliche Dimensionen erweitert und schließt auch das immaterielle Erbe ein. Die UNESCO äußert sich dazu wie folgt:

„Having at one time referred exclusively to the monument remains of cultures, heritage as a concept has gradually come to include new categories such as the intangible, ethnographic or industrial heritage“ (UNESCO 2002a).

Das dem kulturellen Erbekonzept zugrunde liegende Kulturkonzept ist somit ein offenes Kulturkonzept, das die lebendige Kultur (living culture) in gleichem Maße reflektiert, wie es mit der Kultur der Vergangenheit gemacht wird. Der immanente Kulturbegriff der Erbekonzeption ist um die Einsicht in das Gefälle zwischen Wissensgesellschaft sowie Industrie- und Schwellenländer auf der einen Seite und den „last developed countries“ insbesondere in Afrika auf der anderen Seite erweitert. (Albert 2002:31). Appadurei führt an:

"intangible heritage because of its very nature as a map through which humanity interprets, selects, reproduces and disseminates cultural heritage was an important partner of tangible heritage. More important it is a tool through which the tangible heritage could be defined and expressed [thus] transforming inert landscape of objects and monuments turning them into living archives of cultural values'” (Appadurai in Munjeri 2004:18).

In der deutschen und rechtsverbindlichen Fassung der Konvention zum Schutz des immateriellen Kulturerbes (Convention for the Safeguarding of the Intangible Cultural Heritage) wird immaterielles Kulturerbe definiert. Es ist als:

„[...] die Praktiken, Darbietungen, Ausdrucksformen, Kenntnisse und Fähigkeiten - sowie die damit verbundenen Instrumente, Objekte, Artefakte und Kulturräume - zu verstehen, die Gemeinschaften, Gruppen und gegebenenfalls Individuen als Bestandteil ihres Kulturerbes ansehen. Dieses immaterielle Kulturerbe, das von einer Generation an die nächste weitergegeben wird, wird von Gemeinschaften und Gruppen in Auseinandersetzung mit ihrer Umwelt, ihrer Interaktion mit der Natur und ihrer Geschichte ständig neu 
geschaffen und vermittelt innen ein Gefühl von Identität und Kontinuität. Auf diese Weise trägt es zur Förderung des Respekts vor der kulturellen Vielfalt und der menschlichen Kreativität bei. Im Sinne dieser Konvention findet nur dasjenige immaterielle Kulturerbe Berücksichtigung, das mit den bestehenden internationalen Rechtsinstrumenten im Bereich der Menschenrechte sowie der Forderung nach gegenseitiger Achtung zwischen den Gemeinschaften, Gruppen und Individuen und einer nachhaltigen Entwicklung im Einklang steht“42 (UNESCO 2003a:2).

Unter dem Begriff des immateriellen Kulturerbes (Intangible Heritage) fasst die UNESCO eine Fülle nicht-materialisierter Elemente, u.a. vielfältige kulturelle Äußerungen der Menschen von mündlichen Erzähl- und Überlieferungen und Bräuchen über Sprache, Musik, Tanz bis hin zu traditionellen Heilmethoden, Speisezubereitung oder Techniken und Fertigkeiten in der Herstellung etwa von Werkzeugen oder Behausungen (van Zanten 2004:37f; vgl. Blake 2000; vgl. Prott\&O’Keefe 1992). Bemerkenswerterweise werden auch „Instrumente, Objekte [und] Artefakte“ (UNESCO 2003a:2) als immaterielles Kulturerbe gekennzeichnet.

Indem die Konvention in ihrer Definition von immateriellem Kulturerbe die Menschenrechte mit einbezieht und „die gegenseitige Achtung zwischen Gemeinschaften, Gruppen und Individuen“ (UNESCO 2003a:2) hervorhebt, versucht sie Elemente wie Frauenbeschneidung, Apartheid, oder ähnliches eines immateriellen Erbes auszuschalten (van Zanten 2004:38).

Die neue Definition und Kategorisierung kulturellen Erbes ging einher mit der Ausrufung des Jahres 2002 als United Nations Year for Cultural Heritage, sowie der ersten Proklamation von Meisterwerken des mündlichen und nichtmateriellen Erbes der Menschheit (First Proclamation of Masterpieces of the Oral and Intangible Heritage of Humanity; Beier-de Haan 2005:59f).

Alle zwei Jahre können die Mitgliedstaaten der UNESCO besonders erhaltenswerte immaterielle Kulturgüter zur Aufnahme in die Liste der Meisterwerke vorschlagen. Über die Auswahl entscheidet eine internationale Jury (ebd.). Sie bewertet unter anderem folgende Kriterien: herausragender Wert, die Verwurzelung in der jeweiligen Kulturtradition und die Bedeutung für die kulturelle Identität der jeweiligen Gesellschaft oder Gemeinschaft (ebd.).

Weitere Gesichtspunkte sind die Bedeutung des immateriellen Kulturguts als Quelle von Inspiration und interkulturellem Austausch, die heutige kulturelle und soziale

\footnotetext{
${ }^{42}$ Was genau mit einer nachhaltigen Entwicklung verstanden wird, kann leider nicht nachvollzogen werden.
} 
Bedeutung sowie die Qualität in der Ausübung künstlerischer und handwerklicher Fertigkeiten. Auch wird überprüft, ob es sich um ein Zeugnis von gelebter Kultur handelt und inwieweit die betreffende Kulturform vom „Aussterben“ (was immer das bedeuten mag!) bedroht ist. Mit der Aufnahme in die Liste verpflichten sich die jeweiligen Staaten zur dauerhaften Erhaltung ihrer traditionellen Kulturgüter (UNESCO 2003b).

Bereits seit 1993 unterstützt die UNESCO mit ihrem Programm Living Human Treasures Staaten in denen „persons who possess to a very high degree the knowledge and skills required for performing or creating specific elements of the intangible cultural heritage that the Member States have selected as a testimony to their living cultural traditions and to the creative genius of groups, communities and individuals present in their territory" (vgl. UNESCO 2004; vgl. Strasser 2005:70).

Die Preisträger bekommen eine staatliche Auszeichnung verliehen und die Weitergabe deren Wissens, deren Erfahrung und Fertigkeiten an jüngere Traditionen wird durch diverse staatliche Hilfen, wie z.B. finanzielle Hilfen unterstützt (vgl. UNESCO 2004; vgl. Strasser 2005:70).

Der Ausgangspunkt für die internationale Liste des Immateriellen Kulturerbes - die inr Vorbild in der Welterbeliste hat - ist das Masterpieces of the Oral and Intangible Heritage of Humanity Programm. Diese werden von einem Komitee, das sich aus NGO's (z.B. ICTM, IUAES, PICL) zusammensetzt, vergeben. Im Gegensatz zum Welterbe braucht der Antragsteller nicht notwendigerweise ein Staat zu sein, der „Auserkorene“ muss jedoch sein Einverständnis geben (Strasser 2005:70).

Im Jahre 2005 sind 43 „Meisterwerke“ in die Liste hinzugekommen, z.B. Karnevalsbräuche aus Belgien, Märchenerzähler aus der Türkei oder auch Tänze aus aller Welt (UNESCO 2005e).

\subsubsection{Die Großen Ise-Schreine in Japan}

In Ise, einer Stadt im Süden der Insel Honshu (Japan), in der Präfektur Mie nahe der Isebucht gelegen, stehen die Großen Ise-Schreine. Sie sind die am meisten verehrten Schreine Japans und können als eine Facette des immateriellen Kulturerbes (Intangible Cultural Heritage) betrachtet werden (vgl. Munjeri 2004:18).

Ise besteht aus zwei Schreinanlagen mit architektonisch sehr ähnlichen Hauptgebäuden, dem Inneren und dem Äußeren Schrein. Der Innere Schrein von Ise ist 
Amaterasu, der Sonnengottheit und Ahnherrin des Tenno-Geschlechts (das japanische Kaiserhaus) geweiht. Im Inneren Schrein ist ein angeblich aus mythologischer Zeit stammender Spiegel aufbewahrt, der als Hauptheiligtum (shintal) dient und als eine der drei Throninsignien Japans gilt (Tokoro 2001:146f; vgl. Munjeri 2004:15ff). Im Äußeren Schrein, einige Kilometer vom Inneren entfernt, residiert Toyouke, eine Nahrungsgottheit, die wohl ursprünglich als Dienerin der Amaterasu angesehen wurde. Dieser Schrein hat zwar nicht die gleiche Bedeutung wie der Innere Schrein, aber was für den Inneren Schrein gilt, gilt auch für den Äußeren (Tokoro 2001: 147f; vgl. Munjeri 2004:15).

Die Schrein-Anlage von Ise stammt aus dem 5.-6.Jahrhundert. Diese Schreine sind Holzbauten und werden aus religiösen Gründen alle 20 Jahre abgebrannt und exakt gleich neu aufgebaut, der Brauch ist bekannt als shikinen zotai oder shikinen sengu Der jetzige Bau stammt von 1993, der nächste Neubau steht somit 2013 an. Das Hauptgebäude besteht aus Holz und Holzpfeilern, die in den Boden versenkt sind. Ein strohgedecktes Satteldach bedeckt den Bau. Die Gebäude zeichnen sich durch Einfachheit und schlichte Schönheit aus. Der Prozess des Wiederaufbaues benötigt acht Jahre. Die Tradition beinhaltet nicht nur den Wiederaufbau und die Konstruktion an sich, sondern auch das Event als Zeremonie und Übertragung von Wissen (Tokoro 2001:147f; Kirshenblatt-Gimblett 2004:3).

Der komplette Gebäudekomplex wird mit neuem Material wiederaufgebaut. Alle Möbel, Dekorationen und mehr als tausend sakrale Gegenstände und Kleider werden ersetzt. Die Aufbau- und Erneuerungsarbeiten werden durch mehr als hundert Handwerker ausgeführt, die Kenntnis der traditionellen Techniken haben. Diese Techniken werden von Generation zu Generation in einer Art "Lehre“ in zwei bis vier Jahren weiter gereicht.

Die Schreine sind quasi vollkommen „authentisch“ in Design und Technik. Das Holz kommt immer aus dem gleichen Gebiet und der Arbeitsplan für die Niederbrennung und Erneuerung der Schreinanlage ist auf Jahrhunderte vorgefertigt, auch werden für die Konstruktion keine Nägel verwendet, so dass die Handwerker über das Wissen verfügen müssen, wie die einzelnen Teile zusammengesetzt werden (Munjeri 2004:15, Kirshenblatt-Gimblett 2004:3).

Die Unterscheidung zwischen materiellem, immateriellem und einem Naturerbe ist nach Ansicht Kirshenblatt-Gimbletts willkürlich, obwohl ihre Konstruktion systematischem Denken und Historie folgt (ebd. 2004:2). Die prinzipielle Unterscheidung zwi- 
schen materiellem und immateriellem Kulturerbe bei der UNESCO liegt in der Trennung von "things from events (and from knowledge, skills and values)" (2004:3). Nach Ansicht der Autorin sind aber „even things are events“(ebd.). Zur Begründung ihrer Behauptung führt Sie den Philosophen Eveling an, der bemerkt: „A thing is a slow event", das auch wahrnehmbar sei, ,,the perception of change is a function of the relationship between the actual rate of change and the window of our awareness'”(Kirshenblatt-Gimblett 2004:3). Die großen Ise-Schreine sind ein solches Beispiel für einen "slow event" (ebd.).

Nach Ansicht Kirshenblatt-Gimblett gibt es kein materielles Erbe ohne immaterielles Erbe. Letzteres ist nicht nur im materiellen verkörpert, sondern untrennbar mit der materiellen und der sozialen Welt der Menschen verknüpft (2005:18).

Tokoro argumentiert ähnlich. Der Ise-Schrein ist kein „tangible property but a unique example of a living tradition of building whose value is not defined by criteria of the material" (Tokoro 2001:147f).

Die „Symbolhaftigkeit“ und der „Wert“ liegen jenseits des Materiellen im steten Erschaffen des Neuen bzw. im Verständnis von dem, was Buchler (1955:30) umschreibt mit: "The communicative powers of products may far surpass the communicative intent of their producers".

\subsubsection{Weitere Projekte zur Erweiterung des Schutzhorizonts der UNESCO}

Die UNESCO hat seit ihrem sechzigjährigen Bestehen als internationale Organisation eine Vielzahl an Programmen, Konventionen und Empfehlungen verwirklicht. Der Erfolg der Welterbekonvention inspirierte die UNESCO, weitere Programme ins Leben zu rufen.

Unter anderem war dies 1992 ein Programm, das den Schutz des „dokumentarischen Erbes" zum Gegenstand hat. Jene Kulturgüter, die in Archiven, Bibliotheken und in Museen aufbewahrt werden. Mit Hilfe einer Inventarliste wird auf wertvolle Buchbestände, Handschriften, Partituren und seltene Bild-, Ton- und Filmdokumente hingewiesen. Durch die Aufnahme in die Inventarliste wird lediglich eine internationale Auszeichnung ohne finanzielle Unterstützung vorgenommen. Das Register enthält z.B. den Film „Metropolis, den Kodex der Azteken in Mexiko und die neunte Sinfonie 
von Ludwig van Beethoven ${ }^{43}$ (UNESCO 2002b; vgl. Strasser 2005:62; vgl. Hüfner 2005).

Im Herbst 2001 beschloss die Generalkonferenz der UNESCO durch die Konvention zum Schutz des kulturellen Unterwassererbes ${ }^{44}$ (Convention on the Protection of the Underwater Cultural Heritage) eine räumliche Erweiterung des Schutzhorizontes über das Festland hinaus. Die Konvention hat den Schutz und die Erhaltung von historisch bedeutsamen Stätten in Küstennähe zum Gegenstand, da gerade diese leicht von Hobbytauchern und Tauchunternehmungen erreicht werden können.

Gegenwärtig sind der Konvention erst sechs Staate ${ }^{45}$ beigetreten. Das Abkommen bedarf aber mindestens 20 Vertragsstaaten (so in der Konvention festgehalten), um in Kraft zu treten (vgl. Garabello 2003; vgl. Strasser 2005; vgl. Hüfner 2005).

Im Zuge der rasanten Weiterentwicklung von elektronischen und digitalen Medien hat die UNESCO in Zusammenarbeit mit der EU im Jahre 2003 die Charta zur Bewahrung des digitalen Kulturerbes ${ }^{46}$ (Charta on the Preservation of Digital Heritage) realisiert. Der Begriff des Digitalen Erbes umfasst Ton- und Filmaufnahmen sowie alle in der EDV abgespeicherten Daten.

Viele Daten aus den 50'er und 60'er Jahren des letzten Jahrhunderts sind mittlerweile nicht mehr zugänglich. Daher trägt die Charta den Staaten auf, Strategien und Grundsätze zu entwickeln, um den Erhalt des digitalen Erbes zu sichern und den Zugang zu selbigem durch die Öffentlichkeit zu gewährleisten. In diesem Zusammenhang wurde auch der Anspruch nach einer "Computer-,, oder "Digitalarchäologie“ erhoben (vgl. Hüfner 2005, vgl. Strasser 2005:66; vgl. Ross 2000).

Im Zeitalter der Globalisierung und als Reaktion auf die Befürchtung einer Homogenisierung der Weltkulturen (Strasser 2005:66) verabschiedete die UNESCO 2005 das Übereinkommen zum Schutz und zur Förderung der Vielfalt kultureller Ausdrucksformen (Convention on the Protection and Promotion of the Diversity of Cultural Expressions).

\footnotetext{
${ }^{43}$ Die vollständige Liste findet sich unter: http://portal.unesco.org/ci/en/ev.phpURL_ID=1678\&URL_DO $=$ DO_TOPIC\&URL_SECTION=201.html (24.02.2006; 21 Uhr).

${ }^{44}$ Die Konventionstext ist einzusehen unter: http://unesdoc.unesco.org/images/0012/001260/126065e. pdf (24.02.2006; 22 Uhr).

${ }^{45}$ Die Liste der Vertragsstaaten ist einzusehen unter: http://erc.unesco.org/cp/convention_p.asp? language $=\mathrm{E} \& \mathrm{KO}=13520$ (24.02.2006; 22 Uhr).

${ }^{46}$ Die deutsche Fassung der Charta ist einzusehen unter: http://portal.unesco.org/ci/en/file_ download.php/c79eeb450d23f4b30468f2d2afea76f4Charter_ge.pdf (24.02.2006; 22 Uhr).
} 
Das Abkommen stellt Regeln für den Kulturaustausch und den kulturellen Dialog im Zeitalter der Globalisierung auf (UNESCO 2005f: Präambel). Es sieht sowohl die Anerkennung des Menschenrechts des Einzelnen auf kulturelle Selbstbestimmung als auch des Rechts aller Staaten auf eine eigenständige Kulturpolitik vor. Das Abkommen steht in einem engen Zusammenhang mit dem Allgemeinen Übereinkommen über den Handel mit Dienstleistungen (General Agreement on Trade in Services - GATS). Es soll einen ausgewogeneren und umfassenderen Kulturaustausch zwischen den einzelnen Nationen unterstützen, was durch die Sicherung von tragfähigen, lokalen und regionalen Märkten gewährleistet werden soll (UNESCO 2005f; Merkel 2004:44f; vgl. Hüfner 2005; vgl. Strasser 2005:66ff).

\subsection{Erbe, Erben und die Tradition. Phänomenologische Skizzen aus ethnologischer Perspektive}

Im weiteren Kontext dieser Arbeit werden ethnologisch-kulturwissenschaftliche Abstraktionen des kulturellen Erbes im Vordergrund meiner Betrachtungen stehen. Dieser gezielte Fokus ergibt sich aus der Begebenheit, dass die neueren Konventionen der UNESCO explizit auf ein kulturelles Erbe und dessen Diversität, als auf Eigentum abzielen. Ich betrachte dabei unter anderem die Transformation eines kulturellen Erbes, die darauf bedacht ist, den Betrachtungsgegenstand in der Funktion eines kulturelles Gedächtnisses von der lokalen Ebene auf die internationale Ebene zu erheben und als "kulturelles Erbe der Menschheit“ zu klassifizieren. Ich werde dabei ebenso auf den damit inhärenten Wertewandel, die beteiligten Akteure und auf relevante kulturpolitische Ziele der UNESCO eingehen.

Vorerst werde ich, wie bereits in den vorangegangenen Kapiteln einführend die Begriffsgeschichte der hierbei relevanten Termini darstellen. Dies ist zum einen der Begriff des „Erbe“ und „Ererben“ und im weiteren Zusammenhang auch der Begriff der „Tradition“. Die Darstellung des Traditionsbegriffs liegt in der Begebenheit begründet, dass laut Weigel\&Jussen (2005:13) die UNESCO die Begriffe kulturelles Erbe und Tradition nahezu synonym verwendet (vgl. Thum 1985:LVI).

Im Anschluss daran erörtere ich die für das Thema dieser Arbeit relevanten theoretischen Ansätze der kulturwissenschaftlich-ethnologischen Erbeforschung, um das Phänomen eines kulturellen Erbes aus analytischen Zwecken besser zu fassen und 
somit seine Eigentümlichkeiten hervorzuheben. Abschließend stelle ich die Konzeption der UNESCO den vorangegangenen Erkenntnissen gegenüber.

\subsubsection{Eine kurze Etymologie der Begriffe Erbe und Tradition}

„Das Erbe“ als Substantiv im allgemeinen Sinne bedeutet „Hinterlassenschaft eines Verstorbenen" und ist als Rechtsbegriff bereits bei den Kelten bezeugt. Als Ausgangsbedeutung ist hierbei „das einer Waise Gehörige“, also „einer Waise rechtlich zufallendes Besitztum" anzusetzen. Das gemeingermanische Substantiv ist urverwandt mit lat. orbus („,beraubt“) und griech. orb („Waise“). Die Wurzeln des Begriffs liegen in der indogermanischen Sprache und gehen auf orbho- („verwaist; Waise“) zurück (Wermke et al 2001:184; Pfeifer et al 1989:369) ${ }^{47}$.

Im Umgang mit dem Erbe regeln die Lebenden sowohl ihren Austausch mit den Verstorbenen als auch inr Verhältnis zu den noch nicht Geborenen. Obwohl „Erbe“ und "Ererben“ zu den grundlegenden Formen der meisten Gesellschaften zählt, da es Formen der Überlieferung und Weitergabe im historischen und sozialen Kontext organisiert, gehört es nach Ansicht Weigels und Jussens (2005:3; 5; 6) zu den eher vernachlässigten Themen in der Wissenschaft, auch wenn es gegenwärtig - vielleicht gerade deshalb - zum Gegenstand vieler Disziplinen geworden ist ${ }^{48}$.

Das Konzept des „Erbes“ unterscheidet sich von dem der „Tradition“ am deutlichsten durch die Dimension der genealogischen Übertragung zwischen den Generationen bzw. in der Verankerung von Überlieferung mittels der Reproduktion und der familiären Dimension, wohingegen das der Tradition sich durch die Bestände der Überlieferung, durch Schrift, Architektur und Kunst formiert (ebd. 8).

Der Begriff der Tradition ist ein Lehnwort aus dem Lateinischen. Das Fremdwort wurde im 16. Jahrhundert aus lat. traditio („Übergabe; Überlieferung“) entlehnt, welches sich wiederum aus dem lat. dare (,geben“) bildet, aus dem sich auch das heutig verwendete Substantiv „Datum“ („Zeitangabe; Zeitpunkt") bildet (Pfeifer et al 1989:408; Wermke et al 2001:858). Dem Wortsinn nach bezeichnet Tradition „das

\footnotetext{
${ }^{47}$ Das Erbrecht in unserem heutigen allgemeinrechtlichen Verständnis ist im fünften Buch des BGB kodifiziert.

${ }^{48}$ Eine detaillierte Begriffsgeschichte zu Erbe (franz. hérédité) aus kulturwissenschaftlicher Sicht findet sich bei Souriau 1952.
} 
Ensemble des Hergebrachten, Überkommenen und gewohnheitsmäßig Gegebenen“ (Nünning 2004:667).

Erben und Ererben haben eine lange Geschichte und weisen viele Facetten auf Kulturelles Erbe ist, wie bereits in Kapitel 2 dieser Arbeit angemerkt, ein neueres Phänomen. Man stößt bei der Betrachtung von kulturellem Erbe daher zwangsläufig auf die Gestaltung der Beziehung zwischen den Generationen und den Geschlechtern und dem Bemühen, über den eigenen Tod hinaus zu wirken (Lüscher 2002:50).

\subsubsection{Kulturanalytische Ansätze}

Der Ausgangspunkt für ein einführendes Verständnis von Erbe bietet die „Vorbegriffliche" Basissemantik, die, wie oben beschrieben von der innerfamiliären Bewahrung und Tradierung von Vermögen, Grund und Boden und von den damit verbundenen Rechten und Pflichten zeugt (Bodner\&Sohm 2005:12). Ein weiteres Konzept, das vorwiegend aus der Biologie stammt, stellt Erbe auf der Basis eines evolutionistischen Grundgedankens als legitime Vererbbarkeit und Erblichkeit einer "reinen“, vorherrschenden und privilegierten, politischen und symbolisch institutionalisierten Erbschaft dar. Zugleich wird diesem Reinheitsgedanken dann auch ein als „unrein“ bzw. hybrides Erbe gegenübergestellt, das unterdrückt und marginalisiert wird (Bodner\&Sohm 2005:12).

Der Begriff des Erbes zeichnet sich, laut Weigel und Jussen grade dadurch aus, dass er im Unterschied zu Konzepten wie Geschichte, Tradition oder Evolution, die verschiedenen Dimensionen der Überlieferung, vor allem kulturelle, soziale, rechtliche, ökonomische und biologische Aspekte miteinander in einem Zusammenhang verbindet. Dies geschieht, indem Erbe deren Rolle und Zusammenspiel im Verhältnis von Kontinuität und Veränderung und am Übergang von Natur und Kultur organisiert (2005:13f).

Das Thema Erbe spannt einen großen Bogen, das von der Gabe ${ }^{49}$ der Vorfahren an die Nachgeborenen bis zur Vorstellung der Begabung reicht, mit der künftige Geschlechter für eine bessere Welt ausgestattet sein sollen. Indem das erarbeitete Eigentum als eine materialisierte Form zu Lebzeiten verstanden wird, bedeutet Erer-

\footnotetext{
${ }^{49}$ Zum ethnologischen Begriff der Gabe siehe Mauss 2001. Ich werde im weiteren Zusammenhang noch auf die Bedeutung der Gabe im Kontext des Erbes eingehen.
} 
ben in diesem Zusammenhang immer auch, auf Kosten der Vorfahren bzw. Toten zu leben, was im Totenkult und in der Sorge um die Toten seinen Ausdruck findet. Das Erbe als Austausch zwischen Toten und Lebenden verknüpft deshalb auch religionsgeschichtliche und ökonomische Bedeutungen des Tausches (Weigel\&Jussen 2005:5; vgl. Finch\&Wallis 1993: 50ff; vgl. Langbein 2002:15). Egli (1999:436) definiert Erbe daher als „kulturell geprägter, nicht-reziproker diachroner Tausch“50.

Wie die basissemantischen Inhalte des Erbebegriffs zeigen, wird dieser hauptsächlich geprägt durch naturalisierte ${ }^{51}$, verrechtlichte, ökonomisierte und individualisierte Praktiken $^{52}$ (vgl. Weigel\&Jussen 200513; vgl. Langbein 2002:15). Einige Autoren belassen es dabei eher bei einem „reduktionistischen Erbebegriff“ (Langbein 2002:16), der auf ökonomische Ressourcen beschränkt bleibt und das Erben als soziale Praxis erstarren lässt, d.h. die symbolische Bedeutung des Erbens, die für einen kulturanalytischen Ansatz von Interesse ist, wird nicht eruiert (ebd.).

Zudem hat sich eine Politik des kulturellen Erbes etabliert. Im Kontext der Genese der Nationalstaaten wird eine symbolische Politik des kulturellen Erbes entwickelt, für die symbolische Inszenierungen, museale und literarische Formen der Erinnerungspolitik und der Kanonisierung der Traditionsbestände eine zentrale Bedeutung erhalten. In ihr werden die Überlieferungskonzepte von Tradition und kulturellem Erbe nahezu synonym, woraus sich die Hypothese ergibt, dass die Variante des kulturellen Erbes als Traditionskonzept der Moderne betrachtet werden kann (Weigel \&Jussen 2005:13; Mayer 1982:40ff; Csáky\&Sommer 2005:1; s. Kapitel 2).

Gegenüber der Bedeutung der Tradition als Übergabe von Amt, Geschäft und Autorität, wird Tradition - nun verstanden als Modulierung ererbter kultureller Bestände und Gehalte (wie z.B. Lebensform, Weltbildern, Gefüge sozialer Organisation) - zu einer

\footnotetext{
${ }^{50}$ Wie Weigel und Jussen festhalten ist in der deutschsprachigen Erbeforschung das Problem gegeben, dass diese stark fachspezifisch und thematisch ausdifferenziert ist. Die Forschungen zum Erbrecht, zu Vererbungsund Evolutionstheorien, zur Geschichte der Familie, zur Tradition und zum kulturellen Erbe gehen nach Ansicht der Autoren weitestgehend getrennte Wege. Die Autoren heben unter anderem ein „Desinteresse der modernen Ethnologie" hervor (Weigel\&Jussen 2005:6f).

Von einem gänzlichen Desinteresse kann hingegen nicht gesprochen werden, wie diese Arbeit zeigt. Auf Grund des Kausalitätsprinzips von Ursache und Wirkung muss ich jedoch im Umfang dieser Arbeit das Erbekonzept nach seinen thematischen Rahmenbedingungen im Sinne einer causa finalis ausdifferenzieren, um nicht „exorbitanten“ Ausmaßen zu erliegen.

${ }^{51}$ Hierbei sind die Reproduktion der Organismen und die Übertragung von genetischen Merkmalen von Eltern auf ihre Kinder gemeint (vgl. Weigel\&Jussen 2005:11).

${ }^{52}$ Eine detailliertere Darstellung der einzelnen Facetten findet sich bei Weigel und Jussen 2005.
} 
identitätsstiftenden und -stabilisierenden Politik für die Gesellschaft (vgl. Bourdieu 1989). Die unterschiedlichen kulturellen Formen (Texte, Gebäude, Feste, Riten, Sprache etc.) werden durch diese Perspektive zu Monumenten des nationalen und/ oder internationalen Gedächtnisses erhoben (Weigel\&Jussen 2005:13f; vgl. Noras 1994; vgl. Francois\&Schulze 2001). Die Stilisierung als kulturelles Erbe hebt Traditionen auf eine andere Ebene, sie nimmt innen in gewisser Weise ihre Selbstverständlichkeit. Sie ist wie alles, was im Rahmen einer Konjunktur des kulturellen Erbes abläuft, als Prozess der Konstruktion oder Produktion von Kultur zu bewerten (Schneider 2005:2; vgl. Kirshenblatt-Gimblett 1995:367ff).

Bevor ich jedoch weiter auf kulturelles Erbe eingehe, wird es nötig sein die Konzeption von Erbe näher zu betrachten und von seiner bisherigen materiellen Basis zu lösen. Abrams definiert Erbe wie folgt:

"On a general and analytical level the concept of heritage strikes [...] as an ideologically charged textualizing practice intended, with varying degrees of selfconsciousness, to mediate and reimpose order on the destabilizing forces of cultural change. [...] The invention of heritage discourse thus acts as a barometer for social and cultural transformation rather than for stability" (1994:24ff).

Der Autor betont mit seiner Definition die ideologisch strukturale Bedeutung des Erbens und des Erbes in der Praxis, um ungewünschte Folgen und Auswirkungen kulturellen Wandels zu mildern bzw., um einem kulturellen Wandel generell als Ausgleich entgegenzuwirken. Erbe (Heritage) sei als eine Erfindung (Invention) zu betrachten, deren Diskurs als ein „Barometer" für soziale und kulturelle Transformationen deutbar ist. Abrams wendet sich von einer „reinen“ Betrachtung des Erbephänomens als Eigentumsübertragung der Toten auf die Lebenden im Familiärenkontext $a b$ und eröffnet, meiner Perspektive nach, ein erweitertes Spektrum möglicher Interpretationsgehalte, die der Funktion eines Erbes oder des Ererbens gerechter werden, besonders, wenn es um die Funktion eines Erbes geht, dessen Eigenschaft als „kulturell“ vorausgesetzt wird und nicht nur ein erweiterter Rahmen für Eigentumsübertragungen darstellt (s.u.). In diesem Sinne charakterisiert die Autorin Langbein Erben als einen Prozess kultureller Praxis:

„[...], als Prozess also, der hinsichtlich seiner ästhetischen Formen, sozialen Funktionen und symbolischen Bedeutungen untersucht wird. Daraus ergibt sich eine besondere theoretische Herausforderung, die darin besteht, den ökonomistisch [sic.] verengten Erbe- 
Begriff aufzubrechen und den Versuch zu unternehmen, inn kulturwissenschaftlich zu konzeptualisieren“ (2002:13).

Einen weiteren Ansatz lieferte bereits Bourdieu. Der Autor erforscht in der Unterscheidung von ökonomischem, sozialem und kulturellem Kapital die Mechanismen der Reproduktion sozialer Klassen. Die Übertragung materieller Ressourcen ist bei inm nur ein Faktor der Vererbung sozialer Positionen, denn es gibt „kein materielles Erbe, das nicht auch gleichzeitig kulturelles Erbe ist: die Funktion des Familienbesitzes beschränkt sich nicht auf die bloße sachliche Bestätigung der Anciennität [sic.] und Kontinuität des Familiengeschlechtes und darin auf die von der zeitlichen Fortdauer nicht zu trennende Anerkennung ihrer gesellschaftlichen Identität; vielmehr trägt es praktisch zu deren moralisch-geistigen Reproduktion bei, d.h. zur Weitergabe von Werten, Tugenden und Kompetenzen“ (Bourdieu 1989:136f; vgl. Langbein 2002:19).

Bourdieus Erbebegriff bezieht von Anfang an die kulturelle Dimension mit ein. Der Autor reduziert den Erbeprozess nicht auf eine bloße Übertragung von Gütern im Todesfall, sondern konstruiert inn als lebenslangen Prozess der Inkorporation von Wertvorstellungen und kulturellen Mustern (Langbein 2002:19).

Die Wertvorstellungen und Muster werden aber nicht automatisch oder in einem evolutionistisch/genetischen Sinne vererbt, sondern sie werden selektiert, d.h. infolge des kulturellen oder biologischen Todes einer Person steht deren gegenständliche Welt zur Disposition. Diese Welt stellt ein symbolisches Reservoir dar, innerhalb dessen die „Spreu“ vom „Weizen“ getrennt wird: Unbrauchbares oder Ungewolltes wird von Begehrtem oder Nützlichem getrennt, Veräußerbares von Unverkäuflichem geschieden. Die neuen Besitzer bzw. die Erben müssen sich mit dem symbolischen Gehalt bzw. dem Wert, der den Dingen beigemessen wird oder den sie den Dingen beimessen, identifizieren können, um sie als erhaltenswert anzunehmen. In einem „Deklarationsakt“ wird diese Auswahl von seinem neuen Besitzer zum Erbe „geadelt“ (Langbein 2002:220f). Nach dem Ableben Herrn Müllers zum Beispiel hat seine Frau Brunhilde die Aufgabe, aus allem, was vorher ihrem Mann gehörte, jene Elemente auszuwählen, die ihr als erhaltenswert gelten. Sie beschließt, nur den Lieblingsbierkrug ihres verstorbenen Gatten zu erhalten, der Rest wird verschenkt.

Die Dinge des Anderen werden zu den eigenen. Sie werden inkorporiert, d.h. die Dinge aus der Lebenswelt einer anderen Person werden zu Elementen des eigenen Alltags und zum persönlichen Ausdruck und tragen somit zur Identitätsbildung bei. 
Dabei kann es auch zu Modifizierungen des ehemaligen symbolischen Gehaltes kommen, z.B. kann sich ein ehemals in Gebrauch befindlicher Bierkrug nach dem Akt der Vererbung in eine Blumenvase wandeln (ebd.).

War die Kategorie „Erbe(n)“ bisweilen hauptsächlich auf den Versorgungsaspekt und die Übertragung ökonomischer Ressourcen von einer Generation auf die andere ausgerichtet, so lässt sich nun feststellen, dass es sich eher um eine Übernahme als um eine Übertragung handelt. Denn entscheidend sind die Selektionen der Personen oder bestimmter Gruppe, die eine klassifikatorische Ordnung von Dingen, auf Grund des symbolischen Gehaltes, der den Objekten zugewiesen wird, als „Erbe“ benennen. Die Kategorie Erbe umfasst weiterhin das, was aus der Vergangenheit kommt und in die Gegenwart übernommen wird, das Überdauernde, Kontinuierliche. Des weiteren ist Erbe das, was in irgendeiner Weise für wertvoll befunden und daher bewahrt wird. Dadurch verweist diese Kategorie auf Werte, die konstruiert und übertragen bzw. übernommen werden. (Langbein 2002:220ff).

Im folgenden Unterkapitel werde ich in Abgrenzung zur Erbekonzeption theoretische Ansätze zu kulturellem Erbe darstellen. Unter anderem stelle ich dabei die Bedeutung des kulturellen Erbes als Gedächtnisform eines kulturellen Kontextes bzw. einer Gesellschaft dar, deren Funktion nicht nur in der Bildung und Festigung von Identitäten und Legitimitäten zu suchen ist, sondern auch auf Hinsicht einer ökonomisch verwertbaren Ressource. Ich komme dabei noch mal auf die oben dargestellte Konzeptionalisierung von Erbe zurück, da es zwischen beiden Erbformen Schnittmengen gibt, insofern sie dem selben Paradigma folgen. Des weiteren werde ich in diesem Zusammenhang die UNESCO-Konzeptionen den ausgearbeiteten Darlegungen gegenüberstellen und diskutieren.

\subsubsection{Kulturelles Erbe als soziale Konstruktion der Vergangenheit}

Der Begriff „kulturelles Erbe“ signalisiert trotz seiner unterschiedlichsten Konnotationen stets die Verbindung von Wissenschaft, kultureller Identität und kultureller bzw. kulturpolitischer Praxis. Der Begriff, um den es sich hier handeln wird, steht nicht für eine oft restriktiv konservative Auslegung einer bloßen Objektschau und Erhaltung. Vielmehr wird kulturelles Erbe als Prozess verstanden und nicht als Katalog toter und erhaltenswerter Kulturschätze (vgl. Thum 1985:XVII). Der Begriff beinhaltet trotz 
allem weiterhin die materiellen Zeugnisse, aber es sind - wie ich noch darstelle und oben bereits angedeutet habe - Zeugnisse mentaler Prozesse.

Kirschenblatt-Gimblett definiert kulturelles Erbes bzw. „World Heritage [...] as a mode of cultural production that has recourse to the past and produces something new" (2004:1). Als zentrales Argument führt die Autorin die Bemerkung an, dass: „heritage is created through metacultural operations that extend museological values and methods (collection, documentation, preservation, presentation, evaluation, and interpretation) to living persons, their knowledge, practise, artifacts, social worlds, and life space"(2005:1).

Was genau "metacultural" bedeutet und wie es zu verstehen ist, erklärt die Autorin hingegen nicht. Sie erörtert diesbezüglich, dass kulturelle Phänomene und "Träger“ kultureller Elemente durch eine bestimmte Gruppe von Akteuren auf eine Ebene gehoben werden, in der sie zu „metacultural artefact[s]“ transformieren. Durch diesen Prozess entsteht eine neue Beziehung zu jenem "Nachlass“ (ebd. 1f).

Die Intention der Autorin ist, an sich veraltete, nicht mehr lebensfähige, disfunktionale Elemente eines kulturellen Kontextes mit einem neuen Wert auszustatten, mit einem „second life as heritage“ (Kirshenblatt-Gimblett 1998:146f; vgl. Turtinen 2000:4f), indem diese Elemente zu einer Repräsentation ihrer selbst gemacht werden (ebd.; vgl. Schneider 2005:42).

Kulturelles Erbe ist nach Ansich Kirshenblatt-Gimblett etwas Neues, das durch eine sogenannte "heritage industry" erschaffen wird. Vorhandenen Ressourcen, die bisweilen kaum Beachtung fanden oder als obsolet galten, weil sie unter anderem keine ökonomische Verwertbarkeit implizierten, werden durch Interaktionen zwischen Akteuren einer elitären Gruppe neue Werte und symbolische Bedeutungen beigemessen (vgl. Kirschenblatt-Gimblett 1995:369;; vgl. ebd. 2004; 2005; vgl. Langbein 2002; vgl. Turtinen 2000:4f).

Die vielfältigen Bemühungen um das kulturelle Erbe, um dessen Bewahrung, Erhaltung, Wiederbelebung, Unterschutzstellung und Archivierung verändern die betroffenen kulturellen Phänomene in ihrer symbolischen Konsistenz radikal (Schneider 2005:42). Dieser Wertewandel (transvaluation) ist nicht notwendigerweise als negativ zu betrachten, es sollte aber gefragt werden, ob eine derartige Produktion kulturellen Erbes in allen Sphären des Kulturellen, wie in allen Schutzkategorien gleiche Erfolgschancen hat bzw. gleichermaßen Sinn macht und in welcher Art und Weise der Wertewandel sich vollzieht bzw. mit welchen normativen Mustern und Orientierungen 
dieses vermeintliche "Neue" ausgestattet wird. Wie bereits in einem vorhergegangenen Kapitel erwähnt, ist es ein beachtlicher Unterschied, ob man eine Tempelanlage oder eine Andenpopulation unter Schutz stelle. (vgl. Schneider 2005:42; s. Kapitel 4). Kirshenblatt-Gimblett bemerkt hierzu treffend: ",if it is truly vital, it does not need safeguarding; if it is almost dead, safeguarding will not help" (2004:56).

$\mathrm{Ob}$ und inwieweit sich kulturelles Erbe alleinig durch die Neubewertung obsoleter Elemente konstruiert bleibt ebenfalls fragwürdig, wenn man bedenkt, dass die „Erfindung“ bzw. „Erzeugung“ eines kulturellen Erbes in Form einer institutionalisierten Mnemotechnik als Manifestationen eines kulturellen Gedächtnisses interpretierbar ist (vgl. Assmann 1997:52).

Das Erklärungsmuster Assmanns konstatiert im Gegensatz zu Kirshenblatt-Gimbletts Ansatz, dass sich Vergangenheit in symbolischen Figuren manifestiert, die Erinnerung beinhalten. Der Holocaust, die Atombombe auf Hiroshima ${ }^{53}$, Mythen, Tänze, Riten sind solche Erinnerungsfiguren. Für das kulturelle Gedächtnis zählt nach Ansicht Assmanns nicht faktische, sondern nur erinnerte Geschichte. Durch Erinnerung wird Geschichte zum Mythos und dadurch wird sie Wirklichkeit im Sinne einer fortdauernden normativen und formativen Kraft. In der Erinnerung an ihre Geschichte und in der Vergegenwärtigung der fundierten Erinnerungsfiguren vergewissert sich eine Gruppe ihrer Identität (ebd. 52f) und im Falle eines kulturellen Erbes der Menschheit wäre dies - der Logik folgend - die Identität des Menschen als Mensch perse.

Die Gedächtnis- und Erinnerungskunst arbeitet mit imaginären Räumen und mit Zeichensetzungen im natürlichen Raum. Ganze Landschaften können als Medium des kulturellen Gedächtnisses dienen. Sie werden dann weniger durch Zeichen und Symboliken bzw. Denkmäler betont, sondern als Ganzes auf die Stufe eines Zeichens bzw. Symbols gehoben. Als Beispiel wären die totemic landscapes der australischen Aborigines zu nennen. Indem die einzelnen Gruppen der Aborigines an besonderen Festen zu bestimmten Plätzen pilgern, an denen die Erinnerung an die Ahnengeister haftet, von denen sie abstammen, vergewissert sie sich ihrer Gruppenidentität (Assmann 1997:60; vgl. Strehlow 1970).

Sowohl für den Ansatz Kirshenblatt-Gimblett, wie für den Ansatz Assmanns lässt sich, wie ich meine, mit den Worten Schneiders feststellen, „dass das kulturelle Erbe

\footnotetext{
${ }^{53}$ Dadurch, dass die UNESCO den Holocaust und die Atombombe auf Hiroshima als Weltkulturerbe ernannte, wurden sie auf die Ebene der kollektiven Erinnerung in Form von Monumenten transformiert.
} 
eine kulturelle Konstruktion ist, die eine Tendenz zur Virtualisierung aufweist“ (2005:43) oder wie Kirshenblatt-Gimblett betont: „A key to heritage production is their virtuality [...]“(1995:375).

Abschließend stelle ich Schnittmengen zwischen den Erbformen Erbe und kulturelles Erbe auf Grundlage der vorangegangenen Erörterungen dar. Die Kategorien Erbe und kulturelles Erbe bezeichnen Werte, die für die Existenzsicherung und Identitätsbildung für wichtig befunden werden. Diese wiederum können sich entweder ökonomisch, ethisch und/oder lebensweltlich ausformulieren. Kulturelle Muster und normative Orientierungen sind von existentieller Bedeutung (vgl. Langbein 2002:240; vgl. Assmann 1997).

Aufgrund des Übergabeprozesses, der im Falle der Übertragung von ökonomischen Ressourcen eindeutig ist, wird das Erbe in der Literatur auch mit dem Mechanismus der Gabe in Verbindung gebracht. Als inhaltlicher Kern der Dinge erweisen sich die normativen Orientierungen, die im Sozialisationsprozess durch Identifikationsfiguren vermittelt und fest in der Identität der Menschen verankert sind. Um ein Erbe als Gabe zu verstehen, ist die Bewusstwerdung eines geistigen Interaktionsprozesses zwischen den Lebenden (die Erben) und den Toten Voraussetzung (Langbein 2002:240). Dies kann im Falle eines kulturellen Erbes durch institutionalisierte Mnemotechniken geschehen; z.B. Hiroshima, der Ort des Ersteinsatzes USamerikanischer Atombomben (06.08.1945 und Nagasaki 09.08.1945), symbolisiert den Schrecken einer möglichen Vernichtung der globalen Umwelt und Menschheit. Ein Ort moderner „Barbarei“ der durch seine Erhebung zum Weltkulturerbe als Gedächtnisraum global normative Orientierung vermittelt (Prigge 2002:55).

Neben den normativen Orientierungen selbst wird der Mechanismus von Wertevermittlung und -befolgung, im Sinne von Gabe und Gegengabe in die Dinge eingelagert. Der Unterschied zum direkt übereigneten Erbe ist, dass sich der Tausch bei den Dingen im Reich des Imaginären abspielt. Die geerbten Dinge, ob im Kleinen wie im Großen, können als Gabe, als „eine Gabe im Kopf“ verstanden werden (Langbein 2002:241).

Ein weiterer Schnittpunkt betrifft den Vorgang des „Erbens“, in dem all jene Prozesse erfasst werden, die auf Bewahrung zielen und im Dienste der gesellschaftlichen Reproduktion stehen. Es werden kulturelle Präferenzsysteme tradiert, soziale Beziehungen und Familienräume kontinuiert, normative Orientierungen reproduziert. Es handelt sich somit um die Produktion des Dauerhaften (Langbein 2002:241), oder 
wie Kirshenblatt-Gimblett sich im Falle eines kulturellen Erbes äußert, um „something $n e w “(2004: 1)$.

\subsubsection{Kulturelles Erbe und die UNESCO-Konzeptionen}

Im Kontext der Genese der Nationalstaaten wurde eine symbolische Politik des kulturellen Erbes entwickelt, für die symbolische Inszenierungen, museale und literarische Formen der Erinnerungspolitik und der Kanonisierung der Traditionsbestände eine zentrale Bedeutung erhalten (Weigel\&Jussen 2005:13). Der Begriff war laut Thum als Sigel für jene kulturellen Programme gedacht, die unter Umständen vor langer Zeit entstanden sind und sich im Prozess schöpferischer „Kulturarbeit“ immer wieder wandelten und das Denken, Verhalten und Handeln in der Gegenwart und Zukunft prägen (Thum 1985:XVII).

Grundlegend muss beim Kernkonzept des kulturellen Erbes (Cultural Heritage) ebenso wie beim Konzept des kulturellen Eigentums (Cultural Property) festgehalten werden, dass keine allgemein gültige Definition existiert (Blake 2000:62). Die wachsende globale Bedeutung und zunehmende Reichweite des Konzeptes eines kulturellen Erbes erfordern jedoch - laut Blake (2000:63) - eine brauchbare Arbeitsdefinition von dem, was kulturelles Erbe im Kern sei, denn es bestehe Gefahr „of creating future international instruments which extend the range of the term without having settled on a clear understanding of its meaning as employed in existing texts" (ebd.; vgl. Prott\&O’Keefe 1992).

Das Konzept von kulturellem Erbe wurde auf internationaler Ebene aus verschiedenen Wissenschaften, wie z.B. Anthropologie, Ethnologie und Archäologie, importiert, ohne die theoretischen Hintergründe zu beachten (ebd.). Prott folgert daraus für die völkerrechtliche Situation: „While cultural experts of various disciplines have a fairly clear conception of the subject-matter of their study, the legal definition of the cultural heritage is one of the most difficult confronting scholars today“(1989:224).

Es geht dabei nicht um die allgemein verständliche Intention des Konzepts, sondern viel mehr um die Spannbreite der Bedeutungen, die die Begriffskonstellation des kulturellen Erbes exakt umfasst (Blake 2000:64). Im selben Kontext konstatiert Prott: „[...] globalist concepts of the cultural heritage have now been adopted into legal discourse and UNESCO's universalist task in setting international standards is in parallel with such developments as well as globalization of the economy"(1998:222f). 
Betrachtet man einzelne Aspekte, die durch die UNESCO Konzeption des kulturellen Erbes unter Schutz gestellt und revitalisiert werden, so bleibt anzumerken, dass ein Altstadtensemble als ein materielles Kulturerbe auf der einen und eine Ethnie, wie z.B. die Zàpara People aus Ecuador ${ }^{54}$, als immaterielles Kulturerbe auf der anderen Seite, sehr verschieden gelagert sind, zumal letztere Ernennung sehr eigentümlich ist und hier offensichtlich eine Andenpopulation in gewisser Weise zur Schau gestellt wird (vgl. Schneider 2005:42f).

Der Begriff des kulturellen Erbes betrifft auf internationaler Ebene laut Thum die universale Kulturarbeit aller Völker, zielt aber im Endeffekt auf das kulturspezifische Erbe jeder einzelnen Gesellschaft. Die Schlussresolution der UNESCO Konferenz von Mexiko bezieht das kulturelle Erbe deutlich auf die Kulturarbeit der jeweiligen Gesellschaften und anderer spezifischer Gruppen (Thum 1985:XVIII; vgl. UNESCO 1982). Kulturelles Erbe wird in der UNESCO Resolution von Mexiko aus dem Jahre 1982 mit der kulturpolitischen Bedeutung hervorgehoben, die Zusammenarbeit der Völker, Rechte auf Grundfreiheiten, Recht auf Selbstbestimmung, Frieden, Entwicklung durch Selbsterkenntnis, gegenseitiges Verständnis in veränderten, weltweiten sozialen Beziehungen und das Leitbild einer Anti-elitären Demokratie zu verknüpfen bzw. in einen Zusammenhang zu bringen (vgl. UNESCO 1982; Thum 1985:XIX).

Die Ziele der UNESCO sind klar herausgestellt (s. Kapitel 3) und gerade Erbe, wie auch kulturelles Erbe dienen als normative Orientierung, identitätsstiftende Symbolik, formative Kraft und Präferenzsystem.

Das Ideologiepotenzial des Kulturerbe-Begriffs soll und kann nicht geleugnet werden (vgl. Thum 1982:XIX), wenn man allein auf der Ebene der UNESCO bedenkt, welchen historischen und kulturellen Hintergrund die Institution hat, die das Konzept eines kulturellen Erbes als kulturpolitisches Mittel zur Erreichung ihrer Ziele formuliert.

Seit dem Zweiten Weltkrieg hat die UNESCO eine Serie von Erbe-Initiativen ins Leben gerufen. Bereits seit der Hager-Konvention wurde der Begriff des kulturellen Erbes genutzt. Der Fokus selbst lag jedoch auf der Konzeption eines kulturellen Eigentums als materielles unbewegliches Gut (Tangible Immovable Cultural

\footnotetext{
${ }^{54}$ Die Zàpara People sind eine etwa 300 Menschen umfassende Ethnie in Ecuador und Peru, deren Kosmologie, Mythologie, Bräuche, künstlerische Ausdrucksformen und Sprache durch das Programm der „Meisterwerke“ (s.o.) als Intangible Heritage deklariert wurden (vgl. Schneider 2005:42; vgl. UNESCO Liste der Meisterwerke 2001 unter http://www.unesco.org/culture/intangible-heritage/masterpiece.php?lg=en; 26.02.2006; 20 Uhr).
} 
Property; s. Kapitel 4\&6). Erst im weiteren Verlauf wurde dieses Konzept um die Kategorie des materiellen beweglichen Gutes erweitert (Tangible Movable Cultural Property). Mit der Welterbekonvention wandelte sich der konzeptionelle Fokus der UNESCO, die seit dieser Konvention kulturelles Erbe als Leitkonzeption verwendet, unter der sich kulturelles Eigentum eingliedert. Zusätzlich wurde die Konzeption um ein Naturerbe erweitert (vgl. Kirshenblatt-Gimblett 2005:3) ${ }^{55}$.

Als letzte größere Ergänzung der Erbe-Konzeptionen ist die Erweiterung um das geistige/ immaterielle kulturelle Erbe zu sehen (vgl. Kirshenblatt-Gimblett 2005:3; vgl. Turtinen 2000).

Einen Mangel, den alle Konventionen beinhalten ist, dass sie die Hauptelemente inrer Konzeptionen und ausdifferenzierten Bestandteile nicht im Kanon eines bewussten und reflexiven Subjektes definieren (Kirshenblatt-Gimblett 2005:16). Wenn z.B. in den Proklamationen zu Meisterwerken des mündlichen und nichtmateriellen Erbes der Menschheit (Proclamation of Masterpieces of the Oral and Intangible Heritage of Humanity) von einer "collective creation“ oder "[p]erformers, transmitters, and bearers of tradition" (vgl. UNESCO 2001c; 2003c; 2005g; vgl. Kirshenblatt-Gimblett 2005:16) geschrieben steht, werden diese Figuren als ein passives Medium dargestellt, ohne Wille, Intention oder Subjektivität bzw. die Fähigkeit zum Wandel (Kirshenblatt-Gimblett 2005:16).

"Living archive" und „library" sind ähnliche Metaphern, sie stehen nicht für die Rechte einer Person ein oder für das was diese macht, sondern heben deren Rolle als passiver Kulturträger hervor (ebd.).

Alle „heritage interventions“ verändern und beeinflussen die Beziehungen der Menschen in dem Sinne, den sie als symbolischen Gehalt und normative Orientierung beinhalten. Sie verändern das Verständnis und die Perspektive der Menschen, wie diese ihre Lebenswelt ${ }^{56}$ und sich selbst identifizieren und wahrnehmen. Wandel und Veränderung ist ein immanenter Bestandteil jedes kulturellen Kontextes bzw. von

\footnotetext{
${ }^{55}$ In wie weit es sich bei einem Naturerbe nicht eher um ein Kulturerbe handelt, mag hier nicht weiter verfolgt werden. Es sei aber darauf verwiesen, dass die Bedeutung eines Naturraumes für eine bestimmte Gruppe von Menschen sich durch die dem Raum zugewiesene Symbolik, Zeichensetzung und normative Orientierung als Bedeutsam und Erhaltenswert erweist und als identitätsstiftende Ressource gelte (vgl. Assmann 1997). In der Welterbekonvention heißt es hierzu, dass sich Naturräume, die sich u.a. durch "[...] outstanding universal value from the point of view of science, conservation or natural beauty" (UNESCO 1972) auszeichnen, als Naturerbe klassifizierbar seien und somit - wie ich meine - im Prinzip die konzeptionellen Voraussetzungen eines kulturellen Erbes erfüllen.

${ }^{56}$ Zum Lebensweltbegriff s. Husserl 1992 und Habermas 1981.
} 
Kultur, aber jede Maßnahme zur Bewahrung, Konservierung, Schutzmaßnahme und Erhaltung einzelner kultureller Praktiken kann diesen Wandel „erstarren“ lassen bzw. „attempt to slow the rate of change“ (Kirshenblatt-Gimblett 2005: 16; vgl. Sahlins 1999).

Ein weiterer Punkt, der als Schlüsselbegriff in den Erbekonzeptionen der UNESCO immer wiederkehrt ist „humanity“. Als fundamentale Voraussetzungen und Grundlage gelten hierbei Menschen- und kulturelle Rechte, die durch internationale Gerichte im supranationalen Bereich der Verfassungen, Regierungen und bei Ansprüchen in die Urteilsfindung mit einfließen (vgl. Kirshenblatt-Gimblett 2005).

Wird ein kultureller Nachlass bzw. ein Element zum Kulturerbe transformiert, verändert sich dadurch auch die rechtliche Matrix, in der sich das Element vorher befand. Als weitere Grundlage gilt dann „humanity" und somit die Menschen- und kulturellen Rechte, die wiederum auf den Menschenrechten basieren. Das kulturelle Erbe einer einzelnen Gruppe wird zum kulturellen Erbe der Menschheit transformiert. Paradoxerweise wird das Ziel, kulturelle Vielfalt zu bewahren (UNESCO 2005f), durch die Transformation eines einzelnen kulturellen Phänomens in den Stand eines kulturellen Erbes der Menschheit in gewissem Sinne konterkariert (Kirshenblatt-Gimblett 2005:20f). In anderen Worten würde ich sagen: „Von der Vielheit zur Einheit“.

Die Menschheit ist aber - wie Kirshenblatt-Gimblett konstatiert - an sich nicht im gleichen Maßstab zu betrachten, wie jene Gesellschaften, die ein mögliches kulturelles Erbe der Menschheit produzieren. Alle Rechte, die im Prinzip bei einem kulturellen Erbe der Menschheit zum Tragen kommen, sind das Recht auf Zugriff, Verwertung und Konsum und das auch nur durch die Menschen, für die das kulturelle Erbe der Menschheit von Bedeutung und Zugänglich ist (ebd.).

In den Worten und im Sinne Kirshenblatt-Gimbletts, die kulturelles Erbe als Produktion einer Erbe-Industrie versteht, werden kulturelle Elemente von einem Rechtskontext in einen anderen verschoben:

"The enterprise effects a series of shifts, from local to national to world heritage. Or, more recently, from local to world heritage, that is, from a privileged relationship to a cultural good deriving from notions of ancestry, descent, and inheritance to a relationship based on interest, choice, freedom, democratic notions of inclusion, participation, consent, and investment" (2005:21).

Wenn kulturelle Elemente zu einem kulturellen Erbe der Menschheit im Sinne der UNESCO werden, dann wird es im Prinzip für alle Menschen frei zugänglich, d.h. 
aber für jene Gesellschaften, aus deren kulturellen Kontext z.B. etwas als masterpiece of world heritage ausgezeichnet wurde, dass sie keine Eigentums- und Besitzansprüche mehr erheben können (ebd.). In anderen Worten könnte man - wie ich meine - den Prozess der Auszeichnung eines kulturellen Elements zum kulturellen Erbe auch als „Enteignung“ benennen.

Grade der freie Zugang zu derartigen Ressourcen ist ein kulturpolitisches Ziel der UNESCO, wenn die Organisation ökonomische und kulturelle Entwicklung stärken will. In der deutschen Fassung der Allgemeinen Erklärung zur kulturellen Vielfalt heißt es hierzu als Beispiel:

„Angesichts des gegenwärtigen Ungleichgewichts im Transfer und im Austausch von kulturellen Gütern und Dienstleistungen auf globaler Ebene ist es notwendig, die internationale Zusammenarbeit und Solidarität zu verstärken. Dadurch sollen alle Länder, insbesondere die Entwicklungsländer und Schwellenländer, die Möglichkeit erhalten, Kulturindustrien zu entwickeln, die auf nationaler und internationaler Ebene lebens- und wettbewerbsfähig sind“ (UNESCO 2002c).

Der Auftrag der UNESCO besteht unter anderem in der Erhaltung und Schaffung von Frieden, Wohlstand und Entwicklung. Zur Erreichung ihrer Ziele gilt der UNESCO das Konzept des kulturellen Erbes als Mittel, um diese Ziele zu erreichen. Der Faktor einer kulturellen Ökonomie ist in diesem Zusammenhang ein Bestandteil, um diese Ziele zu erreichen ${ }^{57}$. Wird ein kulturelles Element zum Erbe ernannt, folgen dieser Ernennung Investitionen und diese basieren auf ökonomischen Kalkulationen.

Kirshenblatt-Gimblett konstatiert wie folgt:

„While persistence in old life ways may not be economically viable and may well be inconsistent with economic development and with national ideologies, the valorization of those lifeways as heritage (and the integration of heritage into economies of cultural tourism) is economically viable, consistent with economic development theory, and can be brought into line with national ideologies of cultural uniqueness and modernity"(ebd. 2004:4).

Seit dem Pérez de Cuéllar-Bericht von 1996 ist klar, dass die UNESCO Wirtschaft als Teil eines kulturellen Kontextes versteht (Schöfthaler\&Dyroff1997:21ff; vgl. Albert 2002:33f; vgl. Pérez de Cuéllar 1998:7ff; s. Kapitel 4).

\footnotetext{
${ }^{57}$ Die Welt Bank bezieht bei der Vergabe von Krediten mittlerweile „Kultur“ als mögliche ökonomische Ressource mit ein, quasi als „investment opportunity“ (Kirshenblatt-Gimblett 2005:29; vgl. Cernea 2001).
} 
In der Moderne wird die Ökonomie oft als „Maß aller Dinge“"58 interpretiert und gesehen (Lipp 2005:24). In den Kulturwissenschaften, wie auch der Ethnologie sind Formen der Produktion, Distribution und Zirkulation von Gütern nicht unabhängig vom kulturellen Kontext zu betrachten, sondern als konstitutiv für selbigen. Im Gegensatz dazu betrachten Ökonomen Kultur als eine Externalität zum Marktgeschehen, d.h. der Markt ist unabhängig von Kultur zu betrachten: „Externalities are benefits, or costs, of an economic good that are not account for by some kind of market transaction" (Klamer\&Zuihof 1998:29; Kirshenblatt-Gimblett 2005:29). Kultur kann zwar ökonomische Transaktionen beeinflussen, bleibt aber extern zur Funktion des Marktes und wie Kirshenblatt-Gimblett ergänzend bemerkt: „While culture may be an externality in economic theories of markets, economics is not an externality in theories of culture" (2005:31).

In ökonomischen Denksystemen und Theorien wird Kultur als System ideeller und symbolischer Werte generell wenig beachtet. Chancen für Positionierung und Entfaltung bestehen dann, wenn es gelingt, die ideellen und symbolischen Werte kultureller Kontexte auf die Ebene monetären ökonomischen Denkens zu erheben. Dies ist z.B. für den Bereich des mobilen Kulturgutes bis zu einem gewissen Grad gelungen - das Schlagwort „Aktie an der Wand“ wäre ein Beispiel. Authentizität und Originalität des Werks sind dabei erstrangige Marktkriterien (Lipp 2005:25).

Auf dem Gebiet der Ökonomie des kulturellen Erbes spielt der Tourismus eine entscheidende Rolle. Lipp ist der Ansicht, das erhalten wird, was dem Tourismus gefällt und wofür zu zahlen die Menschen bereit sind ${ }^{59}$ bzw., was für die Touristenaufmerk-

\footnotetext{
${ }^{58}$ Die Weltsystemtheorie von Wallerstein wäre ein prägnantes Beispiel dafür, wie theoretisch die euroamerikanische Art und Weise der Ökonomie zum geschichtlichen Bestandteil aller Menschen konstruiert wird (vgl. Wallerstein 1986).

59 Da der ökonomische Wert eines solchen kulturellen "Gutes" aus diversen Gründen nicht einfach durch eine klassische Angebots-Nachrage Kalkulation bestimmt werden kann, haben Frey und Pommerehne (1989) in ihrer Arbeit versucht ökonomische und kulturwissenschaftliche Perspektiven aneinander anzunähern. Die Autoren haben fünf alternative Wertetypen in Bezug auf kulturelles Erbe erarbeitet: „option value“, „existence value“, "bequest value", „prestige value" und „education value“. In ihrem Werk erläutern die Autoren was unter den einzelnen Typen zu verstehen ist und wie sie sich konstituieren. Kirshenblatt-Gimblett zitiert z.B.: "Prestige value - the primary value of being proclaimed a masterpiece of world heritage - refers to the benefits that follow from being endowed with elevated status. This is the logic of award, designations, proclamations, registers, and lists. $[\ldots]$ [P]restige value, [...] ha[s] economic benefits in terms of tourism, for example, [...]. Education value refers to the value of the asset as an educational resource, understood in context of UNESCO as contributing to positive identity, pluralism, dialogue, culture of peace, and economic development' (Kirshenblatt-Gimblett 2005:30; vgl. Frey und Pommerehne 1989, Hervorhebungen im Original).
} 
samkeit entbehrlich erscheint, darauf kann verzichtet werden (2005:25). Eine ökonomisch verordnete Verengung auf Kulturtourismus mündet nach Meinung Lipps in der Begebenheit, das kulturelles Erbe als Massenphänomen am Sightseeing, am Bild, am Klischee, am bloßen Äußerlichen, an der Inszenierung, der Show, am Surrogat, an Fälschung, Übertreibung und Erfindung Genügen findet (ebd. 26).

Es stellt sich also die Frage, was als kulturelles Erbe klassifiziert wird und wer welche Interessen an einem bestimmten kulturellen Erbe hat? Ob es sich dabei ausschließlich um eine Mnemotechnik im Sinne Assmanns handelt, also als Form eines kulturellen Gedächtnisses oder ob es sich dabei um ökonomische Interessen oder kulturpolitische Anliegen im Sinne der UNESCO handelt bleibt offen. Klamer und Zuihof bemerken dazu: "The way in which heritage is founded may not only affect the appraisal of the heritage but may even contribute to the 'creation' of the heritage" (1998:46) während "valorization (awards and plaques) tends to increase valuation, while valuation (discovering that an old table is worth real money) can lead to valorization by calling attention to values other than economic ones. All heritage is created, and economic arrangements are but one factor in shaping it" (Kirshenblatt-Gimblett 2005:30).

Ein weiterer wichtiger Punkt in den UNESCO-Konzeptionen ist die Stärkung der Identität. Mit anderen Worten die Stärkung des Dasein-Selbstverständnisses (Lipp 2005:25). Identität gilt Lipp als Kontinuitätsbegriff mit einer relativen Statik lebenszeitbezogener Identitätshorizonte. Sitten, Bräuche und Gewohnheiten sind ebenso identitätsprägend wie Räume und Zeitbezüge. Gedächtnis und Bild sind dabei wesentliche Identitätsspeicher. Identität als Kontinuitätsbegriff hat nach Ansicht des Autors grade in Zeiten der Kontinuitätsbrüche, der Verwandlungsschübe und Beschleunigung von Veränderungsprozessen Konjunktur (Lipp 2005:26; vgl. Viehoff \&Segers 1999, vgl. Willems\&Hahn 1999).

Lipp interpretiert die Moderne als Epoche der Identitätskrisen, akzentuiert von den beständigen Verlusten an identifikativen Herkunftsbeständen auf der einen und identifikativen Gegenwarts- und Zukunftsoptionen auf der anderen Seite. Identitätsverlust und Identitätsfindung, die Dialektik von Identitätsgewinn und Identitätsverlust, die Aussicht auf Identitätswachstum durch lebensweltliche Entgrenzung werden in Identitätskonzepten thematisiert. Kulturelle Identität zählt, laut Lipp, hierbei zu den übergreifenden und bevorzugten Bezugfeldern. Ein wesentliches Segment kultureller Identität besetzt dabei das kulturelle Erbe. In der Verbindung von Gedächtnis, Bild 
und Kontinuität ist es in einer außerordentlichen Bandbreite vom klassischen Monument bis zum Masterpiece im immateriellen Kontext besonders „identitätsprädestiniert“" (Lipp 2005:26).

Kulturelles Erbe im Sinne der UNESCO ist identitätskonzeptiv wesentlich auf die Konstitution kollektiver Identität ausgerichtet. Die Betonung gemeinsamen Erbes und gemeinsamer Verantwortung sollen das kollektive Bewusstsein im Sinne einer „Kulturgemeinschaft" stärken. Voraussetzung für diese Identitätskonzeption sind Gedächtnisbereitschaft und relative Bildkonstanz (ebd.), d.h. indem bestimmte Traditionen zum kulturellen Erbe stilisiert werden, sollen sie regionale Identität stiften (Schneider 2005:18).

Beide Erfordernisse stehen, nach Ansicht des Autors (s.o.), der Realität postmoderner Lebenswelt diametral gegenüber. Identitätsrelevant sind diese gekennzeichnet durch Auflösung der herkunftsbestimmten Mikro-Lebenswelten, durch „Disembedding“ (Giddens 2004:33ff) bzw. durch die Transformation eines einzelnen kulturellen Phänomens, einer Tradition, in den Stand eines kulturellen Erbes der Menschheit (Kirshenblatt-Gimblett 2005:20f), durch Überschichtung und Vermengung mit den Makro-Lebenswelten der Globalisierung, der "Multinationalen“, des Welthandels und der Weltöffentlichkeit der Medien (Lipp 2005:26f).

Orte, Räume und Zeiten sind in Bewegung (Harvey 1994:48ff), einer „überstrapazierten“ Wahrnehmung entspricht eine „Ästhetik des Verschwindens“ (vgl. Virilio 1986), den überfüllten Gedächtnisspeichern antworten "Strategien des Vergessens“ (Lipp 2005:29; vgl. Schmidt 1986). Identitäten, die sich aus solchen Realitäten zusammensetzen, sind nach Ansicht Lipps, offen, unstet, schweifend und ganz unterschiedlich, gar ambivalent. Zum einen seien die grobkörnigen Raster der Makrostrukturen bestimmend, d.h. dass ein auf mikrostruktureller Differenzierung der Bestände, auf authentischer, am Idealtypus der weitgehenden Unveränderlichkeit des Überlieferten orientierter Umgang mit Kulturerbe unter der identifikativen Reizschwelle fluktuierender transnationaler Befindlichkeiten liegt, woraus sich auch die Unempfindlichkeit respektive Akzeptanz gegenüber den alltäglichen, im Zuge der Zeit liegenden Veränderungen erklärt. In anderen Worten bedeutet dies: solange Angkor-Wat existiert, bleibt Kambodscha identifikativ Kambodscha. Derartiger Anpreisungen bedient sich auch ein Identitätsmarkt (Souvenirs) und eine Identitätsindustrie (heritage industrie). Andererseits werden immer stärker auch das Veränderliche und Fraktale, die Dauerdekonstruktion des Überlieferten und die beständige Überschichtung des Neuen 
identifikativ und kollektiv dominant. Die Etikettierung des Neuen dafür heißen weltoffen, multikulturell, modern, zukunftsorientiert und prosperierend. Demgegenüber befindet sich nach Meinung Lipps, das Identitätsangebot des kulturellen Erbes in der Defensive (Lipp 2005:29f).

Das Identitätskonzept als Teilkonzeption des kulturellen Erbes im Sinne der UNESCO wird von der Politik als "national trademark" auf dem weltweiten Antiquitäten- und Kuriositätenmarkt der Kulturen vereinnahmt. Das kollektive „Wir“, das sich mit den nationalen Welterbestätten verbindet, wird nach Meinung Lipps (2005:29f) politisch nur insoweit gefördert, als es nicht im Widerspruch zu den - weitestgehend ökonomisch dominierten - realpolitischen Identifikationsmustern steht, in den Worten Schneiders (2005:20) sollen „in der gegenwärtigen Aufwertung des kulturellen Erbes [...] bestimmte Traditionen zu einem Wert an sich gemacht werden“. 


\section{Kulturelles Eigentum und kulturelles Erbe. Eine kom- mentierte Zusammenfassung.}

Bereits seit der europäischen Antike ist das Interesse an besonderen kulturellen Elementen aufgrund ihrer Symbolik und ihres innen beigemessenen Wertes belegt. Dieses Interesse und der Gedanke an Erhaltung und Schutz jener Elemente ziehen sich durch alle Epochen Europas. Mit der Etablierung der UNESCO verfestigte sich dieser Gedanke auf institutioneller Ebene mit einem international ausgerichteten Auftrag und Perspektive.

Als eine Institution, die in der Tradition der Aufklärung und des Humanismus steht, versucht sie seit Anbeginn ihres Wirkens auf Grundlage eben dieses ideologischen Paradigmas jene kulturellen Elemente zu schützen, die von herausragender Bedeutung für Nationen und/ oder die gesamte Menschheit sind. Am Anfang galt es der UNESCO vor allem monumentale Immobilien zu schützen, um dann mit der Zeit weitere Kategorien in ihre Konventionen einzubinden. Der Umfang ihrer Schutzkategorien und Interessen im Bezug auf kulturelle Elemente, die als Eigentum oder Erbe eines kulturellen Kontextes oder der ganzen Menschheit klassifizierbar waren und sind, erweiterte sich zunächst auf mobile Elemente bzw. mobiles Kulturgut (im juristischen Sinne). Menschliche Überreste oder archäologische, wie auch ethnographische Gegenstände konnten von nun an unter den Schutz der UNESCO gestellt werden.

Mit dem Aufbrechen der kolonialen Strukturen und den Emanzipationsbestrebungen der ehemalig kolonialisierten Staaten kam es gleichzeitig zu einem generellen Umbruch im Denken der UNESCO. Die alten Kategorien, vom „Stein und Ziegel“Monument oder vom reinen Gegenstand an sich, waren im Angesicht der sich emanzipierenden Staaten obsolet geworden, da sich vermehrt zeigte, dass es mehr kulturelle Identifikationsmuster, Eigentumsformen und Erinnerungstechniken gibt, als die Vorstellungen einer euro-amerikanisch geprägten Organisation bis dahin erlaubten.

In diesem Umbruch und der damit verbundenen Diskussion innerhalb und außerhalb der UNESCO wurde es notwendig, neue Konzepte zu entwerfen. Die Konzeptionen eines kulturellen Eigentums, die sich bis dahin auf der Grundlage eines statischen Kulturbegriffs begründeten, mussten überdacht werden, um anderen kulturellen Anliegen und Mustern gerecht zu werden, und um mutmaßlich nicht offensichtlich als 
ein Hegemon mit imperialistischen Zügen auf dem Gebiet des Wissens und der Kultur zu gelten.

Gerade der Eigentumsbegriff war und ist es, der einer stark historischen euroamerikanischen Prägung unterliegend und verändert werden sollte. Kulturelle Elemente sollten einer vordergründig ökonomischen Verwertbarkeit entzogen und Zugangsrechte zu den jeweiligen Elementen erweitert werden. Ein weiteres Problem beim kulturellen Eigentumsbegriff ist, dass sich nicht alle Kategorien, die man schützen wollte und will, erfassen lassen. Immaterielle Elemente eines kulturellen Kontextes waren schwer zu integrieren. Erst der Erbebegriff war in der Lage, diese und weitere Kategorien aufzunehmen.

Während der in den Konzeptionen der UNESCO hervorgehobene Schwerpunkt bereits seit der Welterbekonvention von 1972 von einem kulturellen Eigentum zu einem kulturellen Erbe gewandelt wurde, so ließ sich der Wandel des bis dahin noch immer vorherrschenden statischen Kulturbegriffs weiter Zeit. Erst Mitte der 80'er Jahre des letzten Jahrhunderts veränderte die UNESCO ihren statischen in einen offendynamischen Kulturbegriff, der es ermöglichte, weitere schützenswerte Kategorien zu erfassen. Im Zuge der Diskussionen, die, bildlich gesprochen, vor allem zwischen der Nord- und Südhemisphäre der Erde auf Ebene der UNESCO geführt wurden, konnte schließlich auf der Grundlage des neuen Kulturbegriffs, der mit der Weltkulturkonferenz von Mexiko Stadt 1982 offiziell Einzug in die UNESCO nahm, die Kategorie des immateriellen Kulturerbes als Bestandteil neuerer Konventionen etabliert werden. Von nun an war es möglich, anstelle alter auf vorwiegend ästhetische Elemente abzielender Konventionen Elemente zu integrieren, die eher durch ihre Prozesshaftigkeit und Wandelbarkeit erfassbar sind und/ oder gänzlich immaterielle Elemente darstellen.

Bis heute gibt es bei der UNESCO jedoch mehr als nur einen Kulturbegriff. Dies bedingt sich durch die Tatsache, dass die älteren Konventionen noch Gültigkeit haben und somit auch die ihnen zugrundeliegende Auffassung von Kultur. Eine weitere Tatsache ist, dass die UNESCO eine politische Institution ist, die kulturpolitische Anliegen verfolgt. Viele Begrifflichkeiten, die in den Konventionen, Empfehlungen und Erklärungen Verwendung finden, werden nicht abstrahiert und voneinander abgegrenzt verwendet. Die unterschiedlichen Konventionen erzeugen zudem eine Vielzahl an unterschiedlichen Erbe- und Eigentumskonzeptionen, so dass man nicht 
von einer einheitlichen Auffassung bei der UNESCO über diese Phänomene ausgehen kann.

Die Begriffskonstellationen des kulturellen Eigentums (Cultural Property) und des kulturellen Erbes (Cultural Heritage) wurden bereits seit der Hager Konvention von 1954 verwendet, aber nicht deutlich in eine mögliche Beziehung gesetzt. Mittlerweile hat sich im Rahmen dieser Arbeit gezeigt, dass kulturelles Eigentum als Teil eines kulturellen Erbes interpretierbar, aber nicht kulturelles Erbe per se ist. In der Analyse der einzelnen Begriffe zeigt sich, dass selbst Eigentum nicht mit kulturellem Eigentum gleichzusetzen ist, auch nicht Erbe mit kulturellem Erbe oder gar kulturelles Eigentum mit kulturellem Erbe.

Woran liegt das? Was sind Unterscheidungsmerkmale und welche Abstraktionsfacetten haben daran ihren Anteil? Es musste auch gefragt werden, wie sich die Begrifflichkeiten der UNESCO auf dem Hintergrund ethnologisch-kulturwissenschaftliche Abstraktionen verhalten?

Die Begriffe des Eigentums und des Besitzes durchdringen unser alltägliches Denken in fast jeglicher Hinsicht. Gerade in Zeiten, in denen Neoliberalismus das liberale Paradigma des Eigentumsbegriffs verstärkt hervorhebt und die Tendenz besitzt, dieses Paradigma global durchzusetzen. Eigentums- und Besitzvorstellungen sind aber abhängig von ihren kulturellen Kontexten, in denen sie Verwendung finden. Eigentum ist im euro-amerikanischen Verständnis als die absolute Verfügungsgewalt über eine Sache definiert. Besitz hingegen nur als die tatsächliche Verfügungsgewalt. Beide Begriffe stehen in einem engen Verhältnis und sind stark system- und gesellschaftsabhängig. Dies war einer der Gründe, warum die UNESCO von ihren kulturellen Eigentumskonzepten abrückte. Eigentum wird im euro-amerikanischen Kontext meist auf Dinge und ökonomische verwertbare Facetten bezogen. Letztendlich bedeutet Eigentum Andere vom Zugang zu etwas auszuschließen.

Im ethnologisch-kulturwissenschaftlichen Sinne entsteht Eigentum durch soziale Beziehungen. Eigentum besteht nicht aus sich selbst heraus, sondern wird erst durch Beziehungen zwischen Menschen erschaffen. Die Formen dieser Beziehungen und auch die Art und Weise dessen, was dann als Eigentum konstruiert wird, sind vielfältig und nicht auf eine bloße Dichotomie von Individual- und Kollektiveigentum zu reduzieren. Eine weitere Eigentümlichkeit von Eigentum ist, dass es meist an der Schnittstelle von Recht und Wirtschaft hervortritt und eine Rolle bei der Konstruktion von sozialer Identität hat. 
Kulturelles Eigentum bedingt sich in Abgrenzung zu Eigentum per se zum einen durch seine identitätsstiftende Bedeutung für eine spezifische Gruppe von Menschen, die einer materiellen oder geistigen Tatsache durch die Zeit hindurch und durch soziale Beziehungen Bedeutung beimessen und dies im Sinne eines „Eigentums" fassen.

Was aber genau dieses Eigentum ist, und wie es sich in seinem kulturellen Kontext durch soziale Beziehungen formiert, ist nicht im selben Verständnis mit dem eines liberalen oder allgemeinen Eigentumsbegriffs zu sehen. Um etwas als bloßes Eigentum zu begreifen, muss keine diachrone Komponente oder eine größere Gruppe von Menschen bedacht werden, die sich als Einheit konstruieren oder konstruiert wird. Eigentum hat, wie ich meine, trotz seiner identitätskonstitutiven Bedeutung einen Charakterzug des flüchtigen, vergänglichen, des Konsums und der Verwertung. Die jeweiligen Schwerpunkte der Begriffe Eigentum und kulturelles Eigentum sind qualitativ verschoben. Dabei ändert sich auch die Akteursebene.

Für die UNESCO ist kulturelles Eigentum eine Addition von ästhetischen und somit materiellen Elementen im Interesse und mit besonderer Bedeutung für die gesamte Menschheit mit der qualitativen Grundlage eines statischen Kulturverständnisses oder als Angelegenheit eines Nationalstaates, der innerhalb gewisser Grenzen selbst definieren kann, was für inn kulturelles Eigentum darstellt. Die betreffenden Gegenstände wurden primär mit dem Schutz des Eigentums assoziiert, statt den Wert und die Symbolhaftigkeit und somit die Bedeutung, die diese Artefakte für eine Gruppe von Menschen oder Gesellschaften haben, hervorzuheben. Kulturelles Eigentum war gedacht als Ressource für die Kulturmärkte dieser Welt und als Konzeption, um diese Güter zu schützen und einen geregelten „Warenverkehr“ zu ermöglichen.

Ein weiteres Anliegen der UNESCO ist die Identitätsbildung von Gruppen aufgrund ihres kulturellen Eigentums und kulturellen Erbes. Diese Identität ist aber nur dann gewollt, wenn sie den humanistischen Idealen der UNESCO folgt. Es kann dabei gefragt werden, ob es sich bei der UNESCO nicht doch um eine Art internationalen Hegemon mit imperialistischen Zügen auf kulturpolitischem Gebiet handelt. Anderen Gesellschaften, die sich nicht mit der euro-amerikanischen Philosophie des Humanismus im Sinne der UNESCO identifizieren, wird Unterstützung und Schutz versagt. Gleichzeitig propagiert die Organisation aber mit ihrer neuesten Konvention den Schutz und die Förderung kultureller Diversität mit dem Anliegen, indigenen Ansprüchen gerecht zu werden. Dabei soll den multiplen Formen von Eigentums- und Erbe- 
konzeptionen, ob geistiger oder materieller Art, auf lokaler und regionaler Ebene internationale und nationale Rahmenbedingungen verschafft werden, aber eben nur, wenn die UNESCO es auf Grundlage ihrer Philosophie vertreten kann.

Im Kontext der Genese der Nationalstaaten wird eine symbolische Politik des kulturellen Erbes entwickelt, für die symbolische Inszenierungen, museale und literarische Formen der Erinnerungspolitik und der Kanonisierung der Traditionsbestände eine zentrale Bedeutung erhalten. In ihr werden die Überlieferungskonzepte von Tradition und kulturellem Erbe nahezu synonym verwendet. Kulturelles Erbe kann dadurch auch als Traditionskonzept der Moderne betrachtet werden. Mit der Welterbekonvention etablierte die UNESCO kulturelles Erbe als Leitkonzept ihrer kulturpolitischen Bestrebungen.

Der Erbebegriff sollte die symbolische Bedeutung der kulturellen Elemente hervorheben, anstatt Erbe als eine ökonomische Ressource zu verstehen. Die Organisation konnte mit den Konventionen zu einem kulturellen Erbe auch die Schwierigkeit umgehen, immaterielles/ geistiges Eigentum zu konzeptionalisieren. Denn gerade der Aspekt eines immateriellen Eigentums ist nicht nur in nationalen Rechtssystemen schwierig zu fassen, sondern generell, wie die aktuellen Diskussionen zeigen.

Das Konzept eines kulturellen Erbes hingegen, das die symbolische Bedeutung und die den kulturellen Elementen immanente normative Orientierung hervorhebt, umgeht diese Schwierigkeit. Zudem wird der Aspekt der Exklusion Anderer gemindert bzw. die Zugangsrechte geweitet.

Andererseits kann man bei der Ernennung eines kulturellen Elements zu einem kulturellen Erbe im Sinne der UNESCO auch, wie ich meine, von einer „Enteignung“ sprechen, da meist kulturelle Elemente, die lokal in ihren kulturellen Kontext eingebunden sind, durch eine Ernennung zum kulturellen Erbe einer Nation oder der ganzen Menschheit aus diesem lokalen Kontext in regionale, nationale oder internationale Kontexte transformiert werden. Dabei werden sie nicht nur der lokalen Kontrolle entzogen, sondern auch mit neuen Werten und normativer Orientierung belegt und entwickeln sich im Sinne Kirshenblatt-Gimbletts zu „something new“.

Der allgemeine Erbebegriff ist hauptsächlich geprägt durch naturalisierte, verrechtlichte, ökonomisierte und individualisierte Praktiken und findet eher als „reduktionistischer Erbebegriff" Verwendung, der auf ökonomische Ressourcen beschränkt bleibt und das Erben als soziale Praxis erstarren lässt. Die symbolische Bedeutung des Erbens wird nicht eruiert. Der Begriff eines kulturellen Erbes hingegen ist darauf 
ausgelegt, eben diese soziale Praxis und symbolische Bedeutung kultureller Elemente besonders hervorzuheben. Letzteres nutzt die UNESCO auch für eine identitätsstiftende und -stabilisierende Kulturpolitik auf internationaler Ebene. Ein weiteres Merkmal von kulturellem Erbe besteht darin, dass es als Manifestation eines kulturellen Gedächtnisses interpretierbar ist, das durch eine Vergegenwärtigung der inm innewohnenden normativen und formativen Kraft Identität erzeugt.

Durch die Transformation eines kulturellen Erbes auf eine andere Ebene bzw. einer "heritage interventions" verändern und beeinflusst dies die Beziehungen der Menschen zu anderen, wie zu sich selbst. Der symbolische Gehalt und die normative Orientierung ändern sich durch die Transformation. Diese verändern dann auch das Verständnis und die Perspektive der Menschen, wie diese ihre Lebenswelt und sich selbst identifizieren und wahrnehmen. Wandel und Veränderung ist ein immanenter Bestandteil jedes kulturellen Kontextes bzw. von Kultur, aber jede Maßnahme zur Bewahrung, Konservierung, Schutzmaßnahme und Erhaltung einzelner kultureller Praktiken kann diesen Wandel „erstarren“ lassen bzw. „attempt to slow the rate of change" (Kirshenblatt-Gimblett 2005:16; vgl. Sahlins 1999).

Obwohl kulturelles Erbe vordergründig durch seine symbolische Bedeutung für Menschen bzw. die gesamte Menschheit gedacht ist, ist es aber immer auch als eine ökonomische Ressource denkbar. Nicht nur die Weltbank hat diese Möglichkeit mittlerweile als ein Kriterium für Kreditvergaben und Schuldenerlass erkannt, auch die UNESCO versucht durch die Förderung von kulturellem Erbe und Tourismus Entwicklung durch monetäre Stärkung in ihrem Sinne zu ermöglichen und internationale Kulturmärkte zu schaffen. Es ist meiner Ansicht nach bedenkenswert, wenn die UNESCO einerseits versucht, durch ihre neuen Konventionen zur kulturellen Vielfalt kulturellen Homogenitätsbestrebungen innerhalb einer globalisierenden Welt entgegenzuwirken und andererseits kulturelles Erbe nutzt, um auf ökonomischem Wege Entwicklung durch monetäre Unterstützung und Tourismus zu fördern. Gerade die Aspekte der Wirtschaft und des Wirtschaften auf Grundlage liberaler Wirtschafttheorien sind doch jene Elemente, die, in mittlerweile vielfältiger Literatur, als Komponenten einer Globalisierung ausgemacht werden, die den Homogenitätscharakter dieses Phänomens bestärken.

Kulturelles Eigentum und kulturelles Erbe sind keine einfach zu fassenden Phänomene. Besondere Gewichtung erhält gerade der Eigentumsbegriff in einer sich immer stärker globalisierenden Welt, in der neoliberales Wirtschaften durch multinationale 
Organisationen auf allen Gebieten artikuliert wird, um ihre ökonomische Interessen durchzusetzen und neue Ressourcen zu erschließen. Insbesondere rücken dabei immer stärker kulturelle Elemente ins Visier einer ökonomischen Verwertbarkeit.

Der Eigentumsbegriff ist aber abhängig von seinem kulturellen Kontext, seiner lebensweltlichen Ausgestaltung und seiner rechtlichen bzw. juristischen Institutionalisierung, d.h. was der Staat oder eine Nation als Eigentum vorgibt, muss im lokalen Kontext keine gelebte Wirklichkeit sein. Dazu kommen dann alle weiteren Instanzen, wie z.B. die der regionalen und internationalen Vorgaben bzw. Ausgestaltungen eines Eigentumsverständnisses. Es ist auch fraglich inwieweit Eigentum letztendlich auf eine reine Subjekt-Objektbeziehung zu reduzieren ist, wenn geistige Elemente oder andere kulturelle Phänomene (z.B. Sklaven- oder Menschenhandel) in ihrem Kontext als Eigentum konstruiert werden. In vielen außereuropäischen Gesellschaften wird zudem keine eindeutige Unterscheidung zwischen "Menschen" und "Dingen“ getroffen. Wie das Beispiel in Kapitel 6.2 zeigt, können „Dinglichkeiten“ nach westlichem Verständnis, in anderen kulturellen Kontexten durchaus als „Persönlichkeiten“ konzeptionalisiert werden.

Die westliche Terminologie des Eigentums und Erbes, mit inrem spezifisch philosophischen Hintergrund wird durch eine zunehmende "time-space compression" (vgl. Harvey 1989) global verbreitet. Ein besseres Verständnis dieser Terminologien, wird für indigene Gesellschaften zur unabdingbaren Voraussetzung, um sich und ihre Erzeugnisse gegen fremde Aneignung, Ausbeutung, Übervorteilung und Vermarktung zu schützen. Dies wiederum setzt einen kulturellen Bewusstwerdungsprozess sowie soziopolitische Organisations- und Handlungsformen voraus, die zum Teil von außen angestoßen, zum Teil auch endogen entstehen können.

Ein interdisziplinär ausgerichtetes Forschungsprojekt könnte einen sinnvollen Beitrag leisten, um die facettenreiche Problematik unterschiedlicher Eigentumskonzeptionen, ob kulturell oder allgemein, vertiefend zu thematisieren.

Im ganzen lässt sich für die kulturpolitische Ebene der UNESCO abschließend sagen, dass das Konzept eines kulturellen Erbes dem eines kulturellen Eigentums vorzuziehen ist, da es anderen Gesellschaften ermöglicht, kulturelle Elemente als symbolische und normative Ressource zu deklarieren, ohne direkt mit liberalen Eigentumsvorstellungen konfrontiert zu werden. 


\section{Bibliographie}

Abrams, J.F. (1994): Lost Frames of Reference: Sightings of History and Memory in Pennsylvania's Documentary Landscape. In: Hufford, M. (ed.): Conserving Culture. A New Discourse on Heritage. Urbana: Uni. of Illinois Press: 24-38

Abramson, A.; D. Theodanopoulos (2000): Land, Law and Environment: Mythical Land, Legal Boundaries. London: Pluto Press

Appadurai, A. (ed.)(1986): The Social Life of Things: Commodities in Cultural Perspective. Cambridge: Cambridge Uni. Press

(1996): Modernity at Large: Cultural Dimensions of Globalization. Minneapolis, London: Uni. Of Minnesota Press

(1998): Globale ethnische Räume. Bemerkungen und Fragen zur Entwicklung einer transnationalen Anthropologie. In: Beck, U. (Hg.): Perspektiven der Weltgesellschaft. Frankfurt/ Main: Suhrkamp: 11-40

Albert, M.-T. (2002): Globalisierung und kulturelle Entwicklung. Das Erbe der Menschheit und seine zeitgemäße Interpretation. In: Ästhetik \& Kommunikation. Heft 119, Jahrgang 33, Berlin: 29-36

Alexander, B. (1990): Archaeology and Looting Make a Volatile Mix. In: Science 250 (4984): 1074-75

Alexander, G.S. (1997): Commodity and Property: Competing Visions of Property in American Legal Thougth 1776-1970. Chicago: Uni. Press

American Society of Appraisers (1989): A Handbook on the Appraisal of Personal Property. Washington D.C.

Anderson, P. (1979): Lineages of the absolutist state. London: NLB

Antons, C. (2004): Intellectual Property Harmonisation within ASEAN and APEC. The Hague:

Kluwer Law International

Asch, M. (1997): Cultural Property and the Question of Underlying Title. In: Nicholas, G.P.; T.D. Andrew (eds.): At a Crossroad: Archaeologists and first Peoples in Canada. Burnaby, B.C.: Archaeology Press: 266-271 
Assmann, J. (1997): Das kulturelle Gedächtnis. Schrift, Erinnerung und politische Identität in frühen Hochkulturen. München: Beck

Assmann, A. (1999a): Erinnerungsräume. Formen und Wandlungen des kulturellen Gedächtnisses. München: Beck

(1999b): Zeit und Tradition. Kulturelle Strategien der Dauer. Köln [u.a.]: Böhlau Verlag

Assmann, A.; D. Harth (Hg.)(1991): Kultur als Lebenswelt und Monument. Frankfurt/ Main: Fischer

Assmann, A; H. Friese (Hg.)( 1999): Identitäten. Erinnerung, Geschichte, Identität 3. Frankfurt/ Main: Suhrkamp

Ballistrazzi, M. (1992): Protecting the National Heritage - The Role of the Police with Regard to Insurance Companies. In: Etudes et dossiers 172: 41-46, Geneva: Geneva Association

Balter, M. (2000): Archaeologists and Rabbits Clash over Human Remains. In: Science 287: 34-35

Bannister, K.P.; G.P. Nicholas (2004): Copyrighting the Past? Emerging Intellectual Property Rights Issues in Archaeology. In: Current anthropology 45 (3): 327-350; Chicago: Uni. Of Chicago Press

Barringer, T.; T. Flynn (eds.)(1998): Colonialism and the Object. Empire, Material Cultures and the Museum. London, New York

Bator, P. (1983): The International Trade in Art. Chicago: Uni. Of Chicago Press

Battaglia, D. (1994): Retaining Reality: some Practical Problems with Objects as Property. In: Man 29: $631-44$

Battiste, M.; J.Y. Henderson (2000): Protecting Indigenous Knowledge and Heritage: A Global Challenge. Saskatoan: Purich Pub.

Bauer,M. (2006): Immaterielle Monopolrechte/ Geistiges Eigentum. In: Uni Protokolle; http://www.uniprotokolle.de/Lexikon/Immaterialg\%FCterrecht.html (29.03.2006; $20 \mathrm{Uhr})$

Baur, F. (1983): Lehrbuch des Sachenrechts. 12 Aufl. München: Beck

Beck, U. (1998): Was ist Globalisierung? Frankfurt/ Main: Suhrkamp 
Beer, B. (2003): Ethnos, Ethnie, Kultur. In: Beer, B.; H. Fischer (Hg.): Ethnoloie. Einführung und Überblick. Berlin: Dietrich Reimer Verlag; 60-72

Beier-de Haan, R. (2005): Jenseits der Dinge. Die Generierung des Intangible Heritage in den ,Gedächtnisorten' Museum und Ausstellung. In: Csáky, M.; M. Sommer (Hg.): Kulturerbe als soziokulturelle Praxis. Innsbruck, Wien, Bozen: Studien Verlag: 57-77

Bell-Krannhals, I. (1990): Haben um zu geben. Eigentum und Besitz auf den Trobriand-Inseln, Papua New Guinea. In: Basler Beiträge zur Ethnologie Bd.31; Basel: Wepf.

Benda-Beckmann, F.; K. Benda-Beckmann; M. Wiber (eds.)(?): Changing Properties of Property. Oxford: Berghahn Books; im Erscheinen

Bengo, R.P. (1987): Protecting Intellectual Property Rights: Issues and Controversies. Washington D.C.: American Enterprise Institute for Public Policy Research

Berger, B.M. (1995): An essay on culture: symbolic structure and social structure. Berkeley: Uni. of California Press

Berger,P.L.; T. Luckmann (1996): Die gesellschaftliche Konstruktion der Wirklichkeit: eine Theorie der Wissenssoziologie. 6. Aufl.; Frankfurt/ Main: Fischer Taschenbuch Verlag.

Bernecker, R. (2002): Anmerkungen zu dreißig Jahren UNESCO-Welterbekonvention. In: Ästhetik \& Kommunikation. Heft 119, Jahrgang 33, Berlin; 23-36

Blake, J. (1996): The protection of the underwater cultural heritage. In: International and Comparative Law Quartely 45: 819

--- (2000): On Defining the Cultural Heritage. In: International and Comparative Law Quarterly 49: $61-85$

(2002): Developing a New Standart-Setting Instrument for the Safeguarding of Intangible Cultural Heritage. Elements for Consideration. Paris

Bodner, R.; K. Sohm (2005): Vorüberlegungen. In: Bricolage: Innsbrucker Zeitschrift für europäische Ethnologie, Bd. 3: 8-34

Bohannen, P. (1967): Law and Warfare: Studies in the Anthropology of Conflict. Garden City, N.Y.: Natural History Press 
Bolliger et al (2004): Geschichte der Welterbe-Idee. In: Hurni, H.; U. Wiesmann; E. Ludi, B. Portner (Hg.): Welterbegebiete im Vergleich. Bern: Center of Developement and Environment

Bostick, W.A. (1977): The Guarding of Cultural Property. Paris: UNESCO

Bourdieu, P. (1989): Die feinen Unterschiede. Kritik der gesellschaftlichen Urteilskraft. Frankfurt/ Main: Suhrkamp

(1994): Zur Soziologie der symbolischen Formen. Frankfurt/ Main: Suhrkamp

(1998): Praktische Vernunft. Zur Theorie des Handelns. Frankfurt/ Main: Suhrkamp

Bourdieu, P. et al (1997): Das Elend der Welt. Zeugnisse und Diagnosen alltäglichen Leidens an der Gesellschaft. Konstanz: UVK Verlag Ges.

(1998): Der Einzige und sein Eigenheim. In: Steinrücke, M. (Hg.): Schriften zu Politik und soziale Wirklichkeit. Bd. 3; Hamburg: VSA Verlag

Boylan, P.J. (1981): Towards a Code of Ethics in Museums. In: Museum Professional Group Transactions 16: 9-32

Breitler, C. et al (1997): La Convention d'UNIDROIT du 24 juin 1995 sur les biens culturels volés ou illicitement exportés. Zürich: Schulthness

Brodersen, K. (1997): Die Sieben Weltwunder: Legendäre Kunst- und Bauwerke der Antike. München: Beck

Brown, M. F. (1998): Can Culture be Copyrighted? In: Current Anthropology 39: 193-222; Chicago: Uni. Of Chicago Press

(2003): Who Owns Native Culture? Cambridge: Harvard Press

(2004): Heritage as Property. In: Humphrey, C.; K. Verdery (eds.): Property in Question: Value Transformation in the Global Economy. New York: Berg: 49-68

Brumann, C. (1999): Writing for Culture. Why a Successful Concept Should Not be Discarded. In: Current Anthropology 40 Supplement: 1-27

Brunner, O. et al (Hg.)(1975): Geschichtliche Grundbegriffe. Historisches Lexikon zur politischsozialen Sprache in Deutschland. Stuttgart: Klett Verlag 
Brush, S. B. (1993): Indigenous Knowledge of Biological Resources and Intellectual Property Rights: the Role of Anthropology. In: American Anthropologist 95: 653-686

(ed.)(1996a): Valuing Local Knowledge: Indigenous People and Intellectual Property Rights. Washington D.C.: Island Press

Burnham, B. (1974): The Protection of Cultural Property: Handbook for National Legislation. Paris: ICOM

(1978): Art Theft, Its Scope, Its Impact, Its Control. New York: International Foundation for Art Research

Cernea, M.M. (2001): Cultural Heritage and Development: A Framework for Action in the Middel East and North Africa. Washington D.C.: World Bank

Cicereo, M.T. (1957): Ciceronis Tusculanarum disputations libri V. [Nachdruck der 5. Aufl.] Suttgart: Teubner

Clément, E. (1993): Illicit Traffic. In: Museum International 45 (4): 61-62

Cohen, M.R. (1927): Property and Sovereignty. In: Cornell Law Quarterly 13: 8-30

Cook, B.F. (1991): The Archaeologist ant the Art Market: Policies and Practice. In: Antiquity 65 (251):533-537

Coombe, R.J. (1993): Cultural and Intellectual Properties: Occupying the Colonial Imagination. In: Political and Legal Anthropology Review 16: 8-15

(1998): The Cultural Life of Intellectual Properties: Authorship, Appropriation, and the Law. Durham, N.C.: Duke Uni. Press

Corsame, G. (ed.)(2005): Heritage, Museum and Galleries: an Introducing Reader. London: Roughledge

Council of Europe (1988): The Art Trade. Report of the Committee on Culture and Education, Parliamentary Assembly. Document 5834

(1993): Architectural Heritage: Inventory and Documentation Methods in Europe. Proceedings of European colloquy organized by the Council of Europe and the French Ministry for Education and Culture-Direction du patrimoine, Nantes, October 28-31, 1992, Strasbourg: Council of Europe 
Crewdsan, R. (1984): Cultural Property - A Fourth Estate. In: Law Society Gazette 18: 126

Csáky, M.; M. Sommer (Hg.)(2005): Kulturerbe als soziokulturelle Praxis. Innsbruck, Wien, Bozen: Studien Verlag,

Cultural Survival Quarterly (1991): The Politics of Ownership. Vol. 15 (3); Summer

Daes, E.J. (1991): Discrimination against Indigenous Peoples: Working Paper on the Question of the Ownership and Control of the Cultural Property of Indigenous Peoples. Sub-commission on Prevention of Discrimination and Protection of Minorities, Commission on Human Rights, United Nations Economic and Social Council

--- (1993): Study on the Protection of the Cultural and Intellectual Property Rights of Indigenous Peoples. E/CN.4/Sub. 2/1993/28 Sub-commission on Prevention and Protection of Minorities, Commission on Human Rights, United Nations Economic and Social Council (1997): Protection of the heritage of indigenous people. In: Human Rights Study Series 10, New York: UN High Commission for Human Rights

Davis, J. (1973): Land and family in Pisticci. In: London School of Economics. Monographs on social anthropology 48; London: Athlon Press

de Araúz, R.T. (1982): Museums and the Containment of Illicit Traffic. In: Museum 28 (4): 134-136

Demsetz, H. (1967): Toward a Theory of Property Rights. In: American Economic Review 57: 347-359

Demsey, M.A. (1995): Protecting the Past: An Interview with Walter Alva. In: Hemispheres 29

Derrida, J. (1972): Die Schrift und die Differenz. 1. Aufl.; Frankfurt/ Main: Suhrkamp

Duff, P.W. (1929): The Personality of an Idol. In: The Cambridge Law Journal 3: 42-48

Dukeminier, J. (2002): Property. Chicago: The BarBri Group

Dukeminier, J.; J.E. Krier (2002): Property. Fifth edition. New York: Aspen Law\&Business

DuMarsais, C.C. (1972): Essay über die Vorurteile : oder Vom Einfluß der Meinungen auf die Sitten und das Glück der Menschen; eine Schrift, die die Verteidigung der Philosophie enthält. 1. Aufl.; Leibzig: Reclam 
Dundes, A. (ed.)(1968): Every Man His Way: Reading in Cultural Anthropology. Englewood Cliffs: Prentice Hall

Durkheim, E. (1908): Die Methode der Soziologie. 4. Aufl. Leipzig: Klinkhardt

Earle, T. (2000): Archaeology, property, and prehistory. In: Annual Review of Anthropology 29: 39-60; Palo Alto, Calif.: Annual Reviews Inc.

Elvert, G. (1999): “Volk”. In: Hirschberger, W. (Hg.): Wörterbuch der Völkerkunde. Berlin: Reimer: 400

Eriksen, T,H. (1997): Do Cultural Island Exist? In: Social Anthropology 1b:1

(2001): A critique of the UNESCO concept of culture. In: Cowan, J.K. et al (eds.): Culture and Rights. Anthropological Perspectives. Cambridge: Uni Press: 127-148

European Union (1994): The Return of Cultural Objects Regulations. Statutory Instruments, No. 501

Eyo, E. (1986): A Threat to National Art Treasures: The Illicit Traffic in Stolen Art. In: Isar, Y.R. (ed.): The Challenge to Our Cultural Heritage: Why Preserve the Past? Paris: UNESCO

Fabrizio, C. (1997): Der unverzichtbare Unterschied. In: UNESCO Kurier 9. Paris: UNESCO

Federal Court of Australia (1989): TERRY YUMBULUL And: RESERVE BANK OF AUSTRALIA; ABORIGINAL ARTISTS AGENCY LIMITED and ANTHONY WALLIS No. D G26 of 1989 FED No. 448 Trade Practices - Aborigines - Copyright 21 IPR 481 http://www.austlii.edu.au/cgibin/disp.pl/au/cases/cth/federal\%5fct/unrep4955.html? query=\%7e+yumbulul (15.02.2006; $22 \mathrm{Uhr})$

Fickentscher, W. (2004): Global intellectual property, competition and cultural traditions. In: Antons, C. (ed.): Intellectual property harmonisation within ASEAN and APEC. 3-9; The Haguo: Kluwer Law International

Finkielkraut, A. (1989): Die Niederlage des Denkens. Reinbeck bei Hamburg: Rowohlt

Fisch, J. (1992): „Zivilisation, Kultur“. In: Brunner, O. (Hg.): Geschichtliche Grundbegriffe. Historisches Lexikon zur politisch-sozialen Sprache in Deutschland. Bd. 7 Stuttgart: Klett-Cotta: 679-774

Fischer, E. (1999): Copyright oder Copywrong?: Geistiges Eigentum, kulturelles Erbe und wirtschaftliche Ausbeutung. In: Zeitschrift für Bibliothekswesen und Bibliographie 46 (6): 515-518 
Fontane, T. (1963): Effi Briest. In: Keitel, W. (Hg.): Theodor Fontane: Sämtliche Werke. 4. Bd. München: Carl Hanser Verlag

Francois, E.; H. Schulze (Hg.)(2001): Deutsche Erinnerungsorte. 3. Bd. München: Beck

Franklin, J.A. (1994): Respecting Ancestors, Archaeologists, and the World Art Community: Enacting a Post-Excavation Cultural Preservation Policy. In: International Journal of Cultural Property 3 (1): 37-60

Fraoua, R. (1986): Convention concernant les mesures à prendre pour interdire et empêcher l'importance, l'exportation, et le transfert de priorité illicites des biens culturels. UNESCO Doc. CC-86/WS/40

Frey, B.S.; W.W. Pommerehne (1989): Muses and Markets: Explorations in the Economics of the Arts. Oxford: Blackwell Press

Friedman, J. (1995): Global System, Globalization and the Parameters of Modernity. In: Featherstone, M. et al (eds.): Global Modernities. London: Sage: 69-90

Frigo, M. (2004): Cultural Property versus Cultural Heritage. A battle of concepts in international law?. In: International Committee of the Red Cross 86: 367-378

Gabig, J.S. (1986): Federal Research Grants: Who owns the Intellectual Property? In: Public Contract Law Journal 6 (1): 187-212

Garabello, R.; T. Scovazzi (eds.)(2001): The protection of the underwater cultural heritage : before and after the 2001 UNESCO. Leiden [u.a.]: Nijhoff

Garducci, G. (1997): La restitution internationale des biens culturels et des objectes d'art. Paris: L.G.D.J.

Geertz, C. (1975): The Interpretation of Cultures. Selected Essays. London: Hutchinson

---- (1969):Religion as a Cultural System. In: Banton, M. (ed.): Anthropological Approaches to the Study of Religion. London: Tavistock:1-46

Giddens, A. (2002): Entfesselte Welt: wie die Globalisierung unser Leben verändert. dt. 1. Ausg.; Frankfurt/ Main: Suhrkamp (2003): Modernity and Self-identity: Self and Society in the Late Modern Age. Cambridge: Polity Press 
Giddens, A. (2004): The Consequences of Modernity. Cambridge: Polity Press

Godelier, M. (1986a): The Mental and the Material: Thought, Economy and Society. London: Verso (1986b): Territory and property in some pre-capitalist societies. In: Godelier, M.: The Mental and the Material: Thought, Economy and Society. London: Verso

Goodeman, N. (1976): Languages of Art. Indianapolis: Hockell Pub. Co.

(1997): Attaching to Things: Property and the Making of an Anti-Cancer Drug. Paper presented to ANT and after workshop, convenor J. Law, Uni. of Keele

Goodenough, W.H. (1957): Cultural Anthropology and Linguistics. In: Garvin, P.L. (ed.): Report of the seventh annual round table meeting on linguistics and language study. Washington: 167-173

(1981): Culture, language, and society. Menlo Park [u.a.]: Benjamin/ Cummings

Graham, I. (1986): Looters Rob Graves and History. In: National Geographic 169 (4): 452-460

Graves, T. (ed.)(1994): Intellectual Property Rights for Indigenous People: a Sourcebook; Oklahoma City: Society for Applied Anthropology

Gray, K. (1991): Property in Thin Air. In: Cambridge Law Journal 50(2): 252-307

Greenfield, J. (1986): The Return of Cultural Treasures. In: Antiquity 60: 29-35

(1989): The Return of Cultural Treasures. Cambridge: Cambridge Uni. Press

Haberland, E. (1981): Überlegungen zum Problem der Restitution von Kulturgütern an die Dritte Welt aus Sicht der Ethnologie. In: Auer, H. (Hg.): Das Museum und die Dritte Welt, 144-153; München

Habermas, J. (1981): Theorie des kommunikativen Handelns. Frankfurt/ Main: Suhrkamp

Hafiz, S.a.D.M. (1877): Der Sänger von Schiras: Hafisische Lieder. Berlin: Hofmann

Halbwachs, M. (1991): Das kollektive Gedächtnis. 4. Aufl. Frankfurt/ Main: Fischer Taschenbuch Verlag

Haller, D. (2005): dtv Atlas. Ethnologie. München: Deutscher Taschenbuch Verlag 
Hann, C.M. (1998a): Property Relations: Renewing the Anthropological Tradition. New York:

Cambridge Uni. Press

(1998b): Property relation: Sharing, Exclusion, Legitimacy. Cambridge: Cambridge Uni. Press

Hann, C. (2004): Besitz und Eigentum. In: Jahrbuch 2004. Max-Planck-Gesellschaft 2004:

Tätigkeitsberichte, Zahlen, Fakten. Halle/Saale: dmv

---- (2005): Property Realtions: the Halle Focus Group 2000-2005. Halle/ Saale: dmv

Hannerz, U. (1987): The World in Creolization. In: Africa 57: 546-559

Hansen, K.P. (1995): Kultur und Kulturwissenschaft: eine Einführung. Tübingen [u.a.]: Vranke

Harrison, S. (1995): Anthropological Perspectives on the Management of Knowledge. In:

Anthropology Today 11 (5): 10-14

Harry, P. (1997): Hybrids of Modernity: Anthropology, the Nation State and the Universal Exhibition. London: Roughledge

Harvey, D. (1989): The Condition of Postmodernity: an Enquiry into the Origins of Cultural Change. Oxford [u.a.]: Blackwell

Harvey, D. (1994): Die Postmoderne und die Verdichtung von Raum und Zeit. In: Kuhlmann, A. (Hg.): Philosophische Ansichten der Kultur der Moderne. Frankfurt/Main: Suhrkamp: 48-78

Haskin, K. (1996): The "awful" idea of accountability: inscribing people into measurement of objects. In: Mursro, R.; J. Mouristsen (eds.): Accountability: Power, Ethos and the Technologies of Managing. London: International Thompson Business Press

Hauser-Schäublin, B. (2001): Von der Kultur in der Natur und der Natur in der Kultur. Eine kritische Reflexion dieses Begriffspaares. In: Brednich, R.W. et al (Hg.): Natur - Kultur : volkskundliche Perspektiven auf Mensch und Umwelt. 32. Kongreß der Deutschen Gesellschaft für Volkskunde in Halle vom 27.9. bis 1.10.1999; Münster [u.a.]: Waxmann: $11-20$

Hays, K.A. (1993): When is a Symbol Archaeologically Meaningful? Meaning, Function, and Prehistoric Visual Arts. In: Yoffee, N.; A. Sherratt (eds.): Archaeological Theory: Who Sets the Agenda? 81-92, Cambridge: Cambridge Uni. Press 
Heidrich, J. (ed.)(1994): Changing Identities. The Transformation of Asian and African Societies under Colonialism. Berlin: Verlag Das arabische Buch

Henare, A. (2003): Artefacts in Theory: Anthropology and Material Culture. In: Cambridge Anthropology 23 (2): 54-66

Herder, J.G. (1965): Ideen zur Philosophie der Menschheit. Berlin: Aufbau Verlag

Hetzel; A. (2001): Interpretation. Jürgen Habermas: Theorie des kommunikativen Handelns. In: Gamm, G. et al (Hg.): Hauptwerke der Sozialphilosophie. Stuttgart Reclam: 249-266

Hirsch, E.; M. O'Hanton (eds.)(1995): The Anthropology of Landscape: Perspectives on Place and Space. Oxford: Clarendon

Hirschberger, W. (Begr.)(1999): Wörterbuch der Völkerkunde. Berlin: Reimer Verlag

Hladig, J. (2003): The Second Protocol to the 1954 Hague Convention and Progress in International Humanitarian Law. In: Museum International 55 (3-4):44-48

Hobsbowm, E.; T. Ranger (eds.)(2003): The Invention of Tradition. 10. Aufl. Cambridge [u.a.]: Cambridge Uni. Press

Hoebel, E.A.; J.D. Jennings (eds.)(1966): Readings in Anthropology. 2. Aufl. New York [u.a.]: McGraw-Hill

Hoebel, E.A. (1958): Man in the primitive world: an introduction to anthropology. New York: McGraw-Hill

Hüfner, K.; W. Reuther (Hg.)(1996): UNESCO Handbuch. Neuwied, Kriftel, Berlin: Luchterhand (2005): UNESCO Handbuch. 2. Aufl. Neuwied, Kriftel; Berlin: Luchterhand

Humphrey, C.; K. Verdery (2004): Property in question: value transformation in the global economy; Oxford: Bery

Huntington, S.P. (1996): Kampf der Kulturen. Die Neugestaltung der Weltpolitik im 21. Jahrhundert. Frankfurt/ Main: Büchergilde Gutenberg

Hurni, H.; U. Wiesmann; E. Ludi, B. Portner (Hg.)(2004): Welterbegebiete im Vergleich. Bern: Center of Developement and Environment 
Husserl, E. (1992): Phänomenologie der Lebenswelt. Ausgewählte Texte Band II. Stuttgart: Reclam.

ICOM (1973): Protection of Cultural Property in Southeast Asia. ICOM meeting of experts; Malacca: ICOM Regional Agency

ICOM (1979): Study of the Principles, Conditions and Means for the Restitution or Return of Cultural Property in View of Reconstituting Dispersed Heritage. In: Museum 31(1): 62-67

---- (1994): Minimum Categories for Museum Object: Proposed Guidelines for an International Standard. Paris

ICOMOS (1985): Ad Hoc Committee of Experts on the Underwater Cultural Heritage. Meeting. 6th. Strasbourg, 1985. Final activity report. Strasbourg, Council of Europe: 34

International Journal of Cultural Property, Berlin und New York, de Gruyter

Isar, Y.R. (1986): The Challenge to our Cultural Heritage: Why Preserve the Past? Paris: UNESCO

Johansen, U. (1992): Materielle oder materialisierte Kultur? In: Zeitschrift für Ethnologie 117: 1-15

Kahn, J.S. (1990): Culture: Demise or Resurrection? In: Critique of Anthropology 9: 5-25

Kant, I. (1968): Anthropologie in pragmatischer Hinsicht. In: Weischedel, W. (Hg.): Werke in 10 Bänden. Bd. 9, Darmstadt: Wiss. Buchgesellschaft

Kasarhérou, E. (1993): Sharing Cultural Heritage. In: Zeitschrift für Ethnologie 118: $23-29$

Kasten, E. (2002): Cultural Heritage: property of individuals, collectivities or humankind? In: Max Planck Institute for Social Anthropology Working Papers 39. Halle/ Saale: MPI (ed.)(2004): Properties of Culture - Culture as Property: Pathways to Reform in postsoviet Siberia; Berlin: Reimer

Kessing, R.M. (1994): Theories of culture revisited. In: Borofsky, R. (Hg): Assessing cultural anthropology. New York [u.a.]: McGraw-Hill :301-310

Keßler, M. (Hg.)(2005): Johann Gottfried Herder: Aspekte seines Lebenswerks. Berlin [u.a.]: Gruyter

King, J.L. (1989): Cultural Property and National Sovereignty. In: Messenger, P. (ed.): The Ethics of Collecting Cultural Property: Whose Culture? Whose Property? Albuquerque: Uni. Of New Mexico Press: 199-208 
Kinder; H.; W. Hilgemann (Hg.)(1998): dtv-Atlas Weltgeschichte. Bd. 1, Aufl. 32: $212 f$

Kirschenblatt-Gimblett, B. (1995): Theorizing Heritage. In: Ethnomusicology 39 (3): 367-380

---- $\quad$ (1998): Destination Culture. Tourism, Museum, and Heritage. Berkeley (u.a.): Uni. of California Press

(2004a): From Ethnology to Heritage: The Role of the Museum. Marseilles:

SIEF Keynot http://www.nyu.edu/classes/bkg/web/SIEF.pdf (27.02.2006; $19 \mathrm{Uhr}$ )

(2004b): Intangible Heritage as metacultural production. In: Museum 221-222 (56): 1-2

(2005): World Heritage and Cultural Economics. In: Karp, I. T al (eds.): Museum Frictions:

Public Cultures/ Global Transformations. http://www.csps.emory.edu/mftoc.htm

(01.05.2006; $16 \mathrm{Uhr})$

Klemm, G.F. (1843): Allgemeine Culturgeschichte der Menschheit nach den beßten Quellen bearbeitet und mit xylographischen Abbildungen der verschiedenen

Nationalphysiognomien, Geräthe, Waffen, Trachten, Kunstproducte u.s.w. versehen.

Leibzig: Teubner

Köster, Thomas (2005): Anthologie. In: Microsoft Encarta 2006 DVD; Microsoft Corporation

Kroeber, A.L.; C. Kluckhorn (1952): Culture: a critical review of concepts and definitions. Papers of the Peabody Museum of American Anthropology and Ethnology. Havard: Cambridge Press

Lafargue, P. (1970): Der Ursprung der Idee des Gerechten und Ungerechten. In: Scheinfuß, K. (Hg.): Paul Lafargue: Vom Ursprung der Ideen - eine Auswahl seiner Schriften 1886-1900. Dresden: Verlag der Kunst

Landeszentrale für politische Bildung (Hg.)(1997): Grundgesetz für die Bundesrepublik Deutschland. 39 Aufl. Mainz: Progress Druck

Langbein, U. (2002): Geerbte Dinge. Soziale Praxis und symbolische Bedeutung des Erbens. Köln [u.a.]: Böhlau Verlag

Latour, B. (2004): Scientific Objects and Legal Objectivity. In: Pottage, A.; M. Mundy (eds.): Law, Anthropology, and the Constitution of the Social: Making Persons and Things. 73-114. Cambridge: Uni. Press 
Lévi-Strauss, C. (1949): Les structures élémentaires de la parenté. Paris: Presses universitaires de France

(1967): Die Mathematik vom Menschen. In: Kursbuch 8: 176-188

(1975): Race and History. In: Kuper, L. (ed.): Race, Science and Society. Paris [u.a.]: UNESCO Press

(1981): Die elementaren Strukturen der Verwandtschaft. Frankfurt/ Main: Suhrkamp

Lévi-Strauss, C.; D. Eribon (1989): Das Nahe und das Ferne: eine Autobiographie in Gesprächen. Frankfurt/ Main: Fischer

Levitt, L. (1987): On Property, Intellectual Property, the Culture of Property and Software Pirating. In: Anthropology of Work Review 8 (1): 7-9

Lewinski, S. v. (ed.)(2004): Indigenous Heritage and Intellectual Property: Genetic Resources, Traditional Knowledge and Folklore; The Hague: Kluwer Law International

Leyton, H. (ed.)(1995): Illicit Traffic in Cultural Property: Museums against Pillage. Amsterdam: Royal Tropical Institute

Lichtenberg, G.C. (1994): „Veränderung“. In: Promies, W. (Hg.): G.C. Lichtenberg. Schriften und Briefe. Bd.1, Frankfurt/ Main: Zweitausendeins

Lipp, Wilfried (2005): Welt-Kultur-Erbe. Im Konflikt der Interessen. In: Csáky, M.; M. Sommer (Hg.): Kulturerbe als soziokulturelle Praxis. Innsbruck, Wien, Bozen: Studien Verlag: 19-30

Liston, D. (ed.)(1993): Museum Security and Protection: A Handbook for Cultural Heritage Institutions. London: ICOM

Losche, D.; S. Walston (1982): Conserving Ethnographic Collections: Problems of Documentation. In: Museum 34 (1):34-36

Lowenthal, C. (1991): Stolen Art: A Positive Move Toward International Harmony. In: Museum News 70: $22-23$

Lutz, C.; L. Abu-Lughod (eds.)(1990): Language and the politics of emotion. Cambridge: Cambridge Uni. Press 
Lüscher, K. (2002): Facetten des Erbes - eine sozilogische Annäherung. In: ebd et al (Hg.) „Was du ererbt von deinen Vätern..." Erben und Vererben. Ethnische, rechtliche, soziologische, politische und psychologische Aspekte eines aktuellen Themas. Tagung vom 08.10.02.2002 in der Evangelischen Akademie Bad Boll . Frankfurt/ Main: epd

Mackensen, L. (1985): Ursprung der Wörter. Etymologisches Wörterbuch der deutschen Sprache. München: Südwest Verlag

MacLean, M.G.H. (ed.)(1993): Cultural Heritage in Asia and the Pacific: Conservation and Policy. Marina del Ray: Getty Conservation Institute

Maurer, B. (2003): Comment: got language? Law, Property, and the Anthropological Imagination. In: American Anthropologist 105: 77-781

Mauss, M. (2001): Die Gabe. Form und Funktion des Austausches in archaischen Gesellschaften. Frankfurt/ Main: Suhrkamp

Mayer, H. (1982): Das "kulturelle Erbe". Vom Sinn und Unsinn eines Klischees. In: Ruppert, W. (Hg.): Erinnerungsarbeit. Geschichte und demokratische Identität in Deutschland. Opladen: 39-54

Mayerhofer, B. (1996): Die Welterbekonvention von 1972. In: Hüfner, K.; W. Reuther (Hg.): UNESCO-Handbuch. Neuwied/ Kriftel/ Berlin: Luchterhand Verlag

McAdams, R. (1971): Illicit International Traffic in Antiquities. In: American Antiquity: ii-iii

McIntosh, R.J., S.K. McIntosh; T. Togola (1989): People Without History. In: Archaeology 42 (1): 74-80, 107

Menzel, E. (1957): UNESCO. Organisation der Vereinten Nationen für Erziehung, Wissenschaft und Kultur (United Nation Educational, Scientific and Cultural Organization). In: Forschungsstelle für Völkerrecht und ausländisches öffentliches Recht der Universität Hamburg (Hg.): Dokumente. Frankfurt/ Main: Wolfgang Metzner Verlag

Merkel, C.M. (2004): UNESCO-Konvention zum Schutz der kulturellen Vielfalt. In: UNESCO heute 51(2): 44-48 
Merryman, J.H. (1986): Two Ways of Thinking about Cultural Property. In: The American Journal of International Law 80: 831-853

(1994): The Nation and the Object. In: International Journal of Cultural Property 3 $(1): 61-76$

(1998): Cultural Property Ethics. In: International Journal of Cultural Property 7(1): $10-20$

(2005): Cultural Property Internationalism. In: International Journal of Cultural Property 12: 11-39

Messenger, P. (1989): The Ethics of Collecting Cultural Property: Whose Culture? Whose Property? Albuquerque: Uni. Of New Mexico Press

Metze-Mangold, V. (2005): Internationale Kulturpolitik in Zeiten der Globalisierung. In: UNESCO heute online 12; http://www.unesco-heute.de/1205/lissabon-rede.htm (22.03.2006; $20 \mathrm{Uhr}$ )

Meyer, K.E. (1973): The Plundered Past. New York: Altheneum Press

Microsoft Corporation (2005): Microsoft Encarta 2006 DVD

Miller, D. (1987): Material Culture and Mass Consumption. Oxford: Basil Blackwell

Monreal, L. (1979): Problems and Possibilities in Recovering Dispersed Cultural Heritage. In: Museum 31 (1): 49-57

Mturi, A.A. (1983): The Return of Cultural Property. In: Antiquity 57: 137-139

Müller, E.W. (1980): Der Begriff "Volk" in der Ethnologie. In: Saeculum 40

Müller, H.P. (Hg.)(1996): Weltsystem und kulturelles Erbe. Gliederung und Dynamik der Entwicklungsländer aus ethnologischer und soziologischer Sicht. Berlin: Reimer

Müller, K.E. (Hg.)(2003): Phänomen Kultur. Perspektiven und Aufgaben der Kulturwissenschaft. Bielefeld: transcript Verlag

Munjeri, D. (2004): Tangible and Intangible Heritage: from difference to concergence, In: Museum International 56 (1-2): 12-19 
Murphy, J.D. (1994): The People's Republic of China and the Illicit Trade in Cultural Property. In: International Journal of Cultural Property 3 (2): 227-242

(1995): Cultural Property Law and Practice in the People's Republic of China. Oxford: Oxford Uni. Press

Nietzsche, F. (1937): Unzeitgemäße Betrachtungen. Zweites Stück: Vom Nutzen und Nachteil der Historie für das Leben. Leibzig: Insel Verlag

Nippold, W. (1954): Die Anfänge des Eigentums bei den Naturvölkern und die Entstehung des Privateigentums. `s-Gravenhage: Mouton\&Co.

Nora, P. (1994): Les lieux e mémoire. 3. Bd. Paris: Gallimard

Nünning, A. (Hg.)(2004): Metzler Lexikon Literatur- und Kulturtheorie. Ansätze - Personen Grundbegriffe. 3. Aufl. Stuttgart, Weimar: Verlag Metzler

Nünning, A; V. Nünning (Hg.)(2003): Konzepte der Kulturwissenschaften. Theoretische Grundlagen - Ansätze - Perspektiven. Stuttgart/ Weimar: Verlag Metzler

O’Keefe, P., L. Prott, L. Law (1984): Law and the Cultural Heritage. London: Butterworths (1993): National Legal Control of Illicit Traffic in Cultural Property. Paris: UNESCO

O'Keefe, P. (1993): Intellectual Property, Cultural Property, Cultural Heritage: Do These Further Indigenous Interests? A paper presented at the First International Conference on the Cultural and Intellectual Property Rights of Indigenous People. Whakatane, Aotearoa, New Zealand

(1994): Feasibility of an International Code of Ethics for dealers in Cultural Property for the Purpose of More Effective Control of Illicit Traffic in Cultural Property. Paris: UNESCO

(1997): Trade in Antiquities. Reducing Destruction and Theft. Paris, London: UNESCO

(2000): Commentary on the UNESCO 1970 Convention on Illicit Traffic. Leicester: Institute of Art and Law

Organisation der Vereinten Nationen für Erziehung, Wissenschaft und Kultur (1970): Übereinkommen über Maßnahmen zum Verbot und zur Verhütung der rechtswidrigen Einfuhr, Ausfuhr und Übereignung von Kulturgut. Paris 
Pannell, S. (1994): 'Mabo and museums: "The indigenous (re-) appropriation of indigenous things"'. In: Oceania 65:18-39

Patent Office (1995): What is Intellectual Property? Newport: Department of Trade and Industry

Pérez de Cuéllar, J. (1998): Our creative diversity: report of the World Commission on Culture and Development UNESCO: Paris

Peya, A. (2002): Die Ausfuhr von Kulturgütern im nationalen und Gemeinschaftsrecht. Frankfurt/ Main: Lang

Pfeifer, W. et al (1989): Etymologisches Wörterbuch des Deutschen. A-G. Berlin: Akademie-Verlag

Pflaum, M. (1967): Die Kultur-Zivilisations-Antithese im Deutschen. In: Knobloch, J. et al (Hg.): Europäische Schlüsselwörter. Wortvergleichende und wortgeschichtliche Studien. Bd. 3 München: Max Huber: 288-427

Platon (1991): Erkenntnistheorie, Dialektik. In: Kunzmann et al (Hg.): dtv-Atlas Philosophie. München: Deutscher Taschenbuch Verlag

Pokorney, D. (2002): Property, Culture and Cultural Property. In: Constellations: an international journal of critical and democratic theory 9 (3): 356-374. Oxford: Blackwell

Portes, E. (1996): ICOM and the Battle Against Illicit Traffic of Cultural Property. In: Museum International 48 (3)

Posey, D.A. (1990): Intellectual Property Rights and fast Compensation for Indigenous Knowledge. In: Anthropology Today 6: 13-16

(1996a): Traditional Resource rights: International Instruments for Protection and Compensation for Indigenous Peoples and Local Communities. Cambridge: IUCN (International Union for the Conservation of Nature)

Posey, D.A.; G. Dutfield (1996b): Beyond Intellectual Property: Toward Traditional Resource Rights for Indigenous People and Local Communities. Ottawa: International Development Research Centre 
Pottage, A. (2004a): Who owns academic knowledge? In: Cambridge Anthropology: a journal of the Department of Social Anthropology 24 (1): 1-20; Cambridge

(2004b): Law, Anthropology and the Constitution of the Social: Making Persons and Things. Cambridge: Cambridge Uni. Press

Prigge, W. (2002): Weltkultur zwischen nationalen Gedächtnissen und kosmopolitischen Erinnerungen. Zum Gedächtnisraum des 20. Jahrhunderts. In: Ästhetik \& Kommunikation. Heft 119, Jahrgang 33, Berlin: 55-64

Prott, L.V.; P.J. O'Keefe (1983): National Control of Illicit Traffic in Cultural Property. UNESCO Doc. CLT-83/WS/16 (1984): Law and the Cultural Heritage. Vol. I, Abingdon: Professional Books Limited (1988): Handbook of National Regulations Concerning the Export of Cultural Property. Paris: UNESCO
(1989): Law and the Cultural Heritage. In: Movement 3, London: Butterworths
(1992): 'Cultural Heritage or Cultural Property'?. In: International Journal of Cultural Property 1: 307-320

Prott, L.V. (1988): Cultural Rights as Peoples' Rights in International Law. In: Crawford, J. (ed.): The Rights of Peoples. Oxford: Clarendon Press: 93-106

\footnotetext{
---- (1989): Problems of Private International Law for the Protection of the Cultural Heritage. In Recueil des cours de l'académiques de droit international de La Haye, 217

(1992): Protecting Art - The Role of UNESCO. In: Etudes et dossiers 172; Geneva: Geneva Association
}

(1996a): Saving the Heritage. UNESCO Action against illicit traffic in Africa. In: Schmidt, P.R.; R.J. Mclntosh (eds.): Plundering Africa's Past.; London: Indiana Uni. Press: 29-44 (1996b): UNESCO and UNIDROIT. A Partnership against Trafficking in Cultural Objects. In: Uniform Law Review 1: 38-45

---- (1998): International Standards for Cultural Heritage. In: UNESCO (Hg.): World Culture Report. Paris: 12-23 
Prott, L.V. (2000a): Commentary on the UNIDROIT Convention. Leicester: Institute of Art and Law.

(2000b): Background Materials on The Protection of the Underwater Cultural Heritage. 2.

Aufl. Paris

Radin, M.J. (1996): Contested Commodities: The Trouble with Trade in Sex, Children, Body Parts and Other Things. Cambridge: Harvard Uni. Press

Ramsauer, T. (2004): Geistiges Eigentum und kulturelle Identität. Eine Untersuchung zum immaterialgüterrechtlichen Schutz autochtoner Schöpfung. München: Verlag C.H. Beck

Raschèr, A.F.G. (2000): Kulturgütertransfer und Globalisierung: UNESCO Konvention 1970 UNIDROIT-Konvention 1995 - EG-Verordnung 3911/92 - EG-Richtlinie 93/8Schweizerisches Recht. Zürich: Schulthess Juristische Medien AG

Reckwitz, A. (2000): Die Transformation der Kulturtheorien: zur Entwicklung eines Theorienprogramms. Weilerswist: Velbrück Wiss.

Renfrew, C. (1993): Collectors Are the Real Looters. In: Archaeology 46 (3): 16-17

---- (1995): Art Fraud: Raiders of the Lost Past. In: Journal of Financial Crime 3 (1): 7-9

Reuther, W. (1996): Die Geschichte der UNESCO: Eine Chronik. In: Hüfner, K.; W. Reuther (Hg.): UNESCO-Handbuch. Neuwied/ Kriftel/ Berlin: Luchterhand Verlag

Reuther; W. (2005): Die Geschichte der UNESCO: Eine Chronik. In: Hüfner, K.; W. Reuther (Hg.): UNESCO-Handbuch. 2. Aufl. Bonn: Uno-Verlag

Riles, A. (2004): Property as legal knowledge: means and ends. In: The Journal of the Royal Anthropological Institute 10 (4): 755-795; London

Richter, G. (1999): Ethics and the rhetoric of culture. In: Adler, H.; J. Hermand (Hg.): Concepts of culture. New York [u.a.]: Lang :163-192

Ritter, J. (1971): Historisches Wörterbuch der Philosophie. Darmstadt: Wiss. Buchges.

Rose, C.M. (1994): Property and Persuasion: Essay on the History, Theory and Rhetoric of Ownership. Boulder: Westview Press

Ross, S. (2000): Changing Trains at Wigan: Digital Preservation and the Future of Scholarship. London: National Preservation Office 
Rowlands, M. (2004): Cultural Rights and Wrongs: Uses of the Concept of Property. In: Humphrey, C.; K. Verdery (eds.): Property in Question: Value Transformation in the Global Economy. New York: Berg: 207-226

Ruppert, W. (Hg.)(1982): Erinnerungsarbeit. Geschichte und demokratische Identität in Deutschland. Opladen: Leske\&Budrich

Sahlins, M. (1994): "A Brief Cultural History of ,Culture". Paper prepared for the World Commission on Culture and Development. Paris: UNESCO

(1999): What is Anthropological Enlightenment? Some Lessons of the Twentieth Century. In: Annual Review of Anthropology 28: i-xxiii

Schiffauer, W. (1997): Kulturalismus vs. Universalismus. Ethnologische Anmerkungen zu einer Debatte. In: ders. (Hg.): Fremde in der Stadt. Zehn Essays über Kultur und Differenz. Frankfurt/ Main: Suhrkamp: 144-156

Schmidt, B. (1986): Postmoderne-Strategien des Vergessens. Darmstadt [u.a.]: Luchterhand

Schmidt, P.R. (1996): The Human Rights to a Cultural Heritage. African Application. In: Schmidt, P.R.; R.J. Mclntosh (eds.): Plundering Africa's Past. London: Indiana Uni. Press

Schneider, I. (2005a): Zur Semantik des kulturellen Erbes. In: Bricolage. Innsbrucker Zeitschrift für europäische Ethnologie, Bd. 3

(2005b): Wiederkehr der Tradition? Zu einigen Aspekten der Gegenwärtigen Konjunktur des kulturellen Erbes. In: Österreichische Zeitschrift für Volkskunde Bd. 108: 1-20

Schoenfelder, M. (1999): „Idol“. In: Hirschberger, W. (Begr.): Wörterbuch der Völkerkunde. Berlin: Reimer Verlag; 183

Schöfthaler, T.; H.D. Dyroff (1997): Unsere kreative Vielfalt: Bericht der Weltkommission „Kultur und Entwicklung"; 2. Ausg. Bonn: Deutsche UNESCO-Kommission e.V.

Scholz, P.O. (1994): Abu Simbel : in Stein verewigte Herrschaftsidee. Köln: DuMont

(2006): Nubien: geheimnisvolles Goldland der Ägypter. Stuttgart: Theiss

Schott, R. (1970): Die Funktion des Rechts in primitiven Gesellschaften. In: Jahrbuch für Rechtssoziologie und Rechtstheorie. Bielefeld: Bertelsmanns Universitätsverlag: 107-174 
Schwab, D. (1975): „Eigentum“. In: Brunner, O. et al (Hg.): Geschichtliche Grundbegriffe. Historisches Lexikon zur politisch-sozialen Sprache in Deutschland. Bd.2; Stuttgart: Klett-Cotta: $65-115$

Serageldin, I; E. Shluger et al (eds.)(2001): Historical Cities and Sacred Sites: Cultural roots for Urban Futures. Washington D.C.: International Bank for reconstruction/ World Bank

Shennon, S. (ed.)(1989): Archaeological Approaches to Cultural Identity. London: Unwin Hyman

Shyllon, F. (1998): International Standards: an African perspective. In: UNESCO (Hg.): World Culture Report. Paris

Siehr, K. (1992): Preliminary Draft UNIDROIT Convention on Stolen or Illegally Exported Cultural Object. In: International Journal of Cultural Property 1 (1): 252-255

Sledge, J. (1993): UNESCO-ICOM Museum Information Centre. In: ICOM News 46 (4): 14-15

Sorlien, P.C. (1989): Valuing Research and Analysis. A Handbook on the Appraisal of Personal Property. Washington D.C.: American Society of Appraisers

Soucy, C.; J.N. Smyth (eds.)(1994): The Appraisal of Personal Property: Principles, Theories, and Practice Methods for the Professional Appraiser. Washington D.C.: American Society of Appraisers

Souriau, E. (1952): Réflexions sur la notion d'hérédité. In: Revue philosophique de la France et de l'étranger: 165-186

Staunton, I.; M. McCartney (eds.)(1981): Lost Heritage. Report of the Symposium on the Return of Cultural Property. London: Commonwealth Arts Association

Steinbacher, F. (1976): Kultur: Begriff - Theorie - Funktion. Stuttgart: Kohlhammer

Stolcke, V. (1995): Talking culture: new boundaries, new rhetoric's of exclusion in Europe. In: Current Anthropology 36: 1-24

Strasser, P. (2005): Das kulturelle Erbe auf internationalem Parkett. In: Bricolage: Innsbrucker Zeitschrift für europäische Ethnologie, Bd. 3: 52-77 
Strathern, M. (1990): Artefacts of History: Events and the Interpretation of Images. In: J. Siikaka (ed.): Culture and History in the Pacific. Helsinki: Suomen Antropologinen Seura, Transaction No. 27

---- (1996): Potential Property. Intellectual Rights and Property in Person. In: Social Anthropology 4 (1): 17-32

(1999a): What is Intellectual Property after? In: The Sociological Review 9: 156-180

(1999b): Property, Substance and Effect. Anthropological Essays on Persons and Things. London, New Brunswick: The Athlone Press

(2000): Audit Cultures: Anthropological Studies in Accountability, Ethics, and the Academy. London: Routledge

(2004a): Social Property: an Interdisciplinary Experiment. In: Political and Legal Anthropology Review 27(1): 23-50

(2004b): The Whole Person and its Artefacts. In: Annual Review of Anthropology 33 (2): 1-20, Palo Alto Calif.: Annual Reviews Inc.

Strehlow, T.G.H. (1970): Geography and Totemic Landscape in Central Australia: A Functional Study. In: Berndt, R. (ed.): Australian Aboriginal Anthropology, A.I.A.S., Canberra: 92-140.

Taboroff, J. (1992): Bringing Cultural Heritage into the Development Agenda: Summary Findings of a Report of Cultural Heritage in Environment Assessment in Sub-Sahara Africa. In: Seragelin, I; J. Taboroff (eds.): Culture and Development in Africa 2: 322-339; Washington D.C.: World Bank

Thomas, N. (1991): Entangled Objects: Exchange, Material Culture and Colonialism in the Pacific. Cambridge: Harvard Uni. Press

Thompson, M. (1979): Rubbish Theory: The Creation and Destruction of Value. Oxford [u.a.]: Oxford Uni. Press

Thornes, R. (1995): Protecting Cultural Objects Through International Documentation Standards: A Preliminary Survey. Santa Monica: Getty Art History Information Program

Thum, B. (1985): Gegenwart als kulturelles Erbe: ein Beitrag der Germanistik zur Kulturwissenschaft deutschsprachiger Länder. München ludicum Verlag 
Tokoro, I. (2001): The Shrine of Ise: the Preservation by Removal and Renewal. In: Serageldin, I; E. Shluger et al (eds.): Historical Cities and Sacred Sites: Cultural roots for Urban Futures. Washington D.C.: International Bank for reconstruction/ World Bank; 147-152

Train, Russel (2002): The World Heritage - A Vision for the Future. Remarks of the Honourable Russel E. Train on the WHC 30. Anniversary. 16.11.2002 Venice, Italy. http://whc.unesco.org/venice2002/speeches/pdf/train.pdf (14.02.2006; $20 \mathrm{Uhr}$ )

Tubb, K.W. (ed.)(1995): Antiquities Trade or Betrayed: Legal, Ethical and Conservation. London: Archetyp

Turtinen, J. (2000): Globalising Heritage: On UNESCO and the Transnational Construction of a World Heritage. In: UNESCO, Score Rapportserie 12: 8-19

Tylor, E.B. (1871a): Primitive Culture : researches into the development of mythology, philosophy, religion, language, art and custom. London: Murray

---- (1871b): Die Anfänge der Cultur : Untersuchungen über die Entwicklung der Mythologie, Philosophie, Religion, Kunst und Sitte. Leipzig: Winter

(1958): The Origins of Culture. [Zuerst: 1871] London: Murray

Uhl, H. (2002): Zwischen Pathosformel und Baustelle: Kultur und europäische Identität. In: Ästhetik \& Kommunikation. Heft 119, Jahrgang 33, Berlin: 129-147

UN (1945): Charta der UN. http://www.runic-europe.org/german/charta/charta.htm (27.01.2006; $15 \mathrm{Uhr})$

---- (2006): Mitgliederliste. http://www.un.org/Overview/unmember.html (27.01.2006; 16 Uhr)

UNESCO (1946): Constitution of the UNESCO, Publ. Nr. 367: 13

(1954): Convention on the Protection of Cultural Property in the Event of Armed Conflict with Regulations for the Execution of the Convention. http://unesdoc.unesco.org/images/0008/000824/082464mb.pdf (04.02.2006; 20 Uhr)

(1956): Recommendation on the conduct of archaeological excavations. Paris: UNESCO

---- (1960): Recommendation concerning the most Effective Means of Rendering Museums Accessible to Everyone. Paris: UNESCO 14. Dez. 1960 
UNESCO (1962): Recommendation concerning the Safeguarding of the Beauty and Character of Landscape and Sites. Paris: UNESCO 11. Dez. 1962

(1968): Recommendation concerning the Preservation of Cultural Property Endangered by Public or Private Works. Paris: UNESCO 19. Nov. 1968

(1970a): Convention on the Means of Prohibiting and Preventing the Illicit Import, Export, and Transfer of Ownership of Cultural Property; Paris: UNESCO

(1970b): Übereinkommen über Maßnahmen zum Verbot und zur Verhütung der rechtswidrigen Einfuhr, Ausfuhr und Übereignung von Kulturgut. Paris: UNESCO

(1970c): Cultural Rights as Human Rights. Studies and Documents on Cultural Policies. No. 3; Paris: UNESCO

(1972): Welterbekonvention. Paris http://www.unesco.de/c_bibliothek/welterbe konvention.htm (01.05.2006; $16 \mathrm{Uhr})$

(1976): Recommendation Concerning the International Exchange of Cultural Property. http://www.unesco.org/culture/laws/exchange/html_eng/page1.shtml (19.04.2006; 15 Uhr)

(1978a): Recommendation for the Protection of Movable Cultural Property. UNESCO: Paris 28. Nov. 1978

(1978b): Statutes of the [UNESCO] Intergovernmental Committee for Promoting the Return of Cultural Property to its Countries of Origin or its Restitution in Case of Illicit Appropriation. http://www.unesco.org/culture/laws/committee/html_eng/statuts.shtml (11.02.2006; $21 \mathrm{Uhr})$

(1979): A plea for the Return of an Irreplaceable Cultural heritage to those who Created It. In: UNESCO Doc.SHC-76/CONF.615.5. UNESCO: Paris

(1982): Mexico City Declaration on Cultural Policies, World Conference on Cultural Policies Mexico City, 26 July - 6 August 1982; http://www.unesco.org/culture/laws/ mexico/html_eng/page1.shtml (20.03.2006; 22 Uhr)

(1989): Recommondation on the Safeguarding of Traditional Culture and Folklore. http://unesdoc.unesco.org/images/0008/000846/084696e.pdf\#page $=242$ (23.02.2006;16 Uhr) 
UNESCO (1993): Report by the Intergovernmental Committee for Promoting the Return of Cultural Property to its Countries of Origin or its Restitution in Case of Illicit Appropriation on its Activities; Paris: UNESCO

---- (1994a): Expert meeting on the 'Global Strategy' and thematic studies for a representive World Heritage List. WHC-94/CONF.003/INF UNESCO

(1994b): Nara Document on Authenticity, Report of the Experts Meeting. WHC94/CONF.003/INF.008 Phuket/ Thailand: UNESCO

(1996): First Proclamation of Masterpieces of the Oral and Intangible Heritage of Humanity. http://portal.unesco.org/culture/en/ev.php-URL_ID=2226\&URL_DO=DO_TOPIC\& URL_SECTION=201.html (01.05.2006; 20 Uhr)

(1997a): Preventing the Illicit Traffic in Cultural Property. A Resource Handbook for the Implementation of the 1970 UNESCO Convention. Paris: UNESCO

(1997b): Unsere Kreative Vielfalt. Bonn: UNESCO

(1998): World Culture Report. Paris: UNESCO

(1999): International Code of Ethics for Dealers in Cultural Property. http://portal.unesco.org/culture/en/file_download.php/cd6248b583cb3a9376ea41c9a635 a03bethics.doc (11.02.2006; $22 \mathrm{Uhr})$

(2001a): Verfassung der UNESCO. http://www.unsco.de (26.01.2006; 15 Uhr)

(2001b): Universal Declaration on Cultural Diversity. Paris: UNESCO; http://unesdoc.unesco.org/images/0012/001271/127160m.pdf (21.03.2006; $19 \mathrm{Uhr}$ )

(2001c): First Proclamation of Masterpieces of the Oral and Intangible Heritage of Humanity. UNESCO http://portal.unesco.org/culture/en/ev.php-URL_ID=2226\&URL_DO= DO_TOPIC\&URL_SECTION=201.html (01.05.2006; 20 Uhr)

---- (2002a): Diversity, Dialogue and Responsibility. UNESCO

http://portal.unesco.org/culture/en/ev.php@URL_ID=1473\&URL_DO=DO_TOPIC\& URL_SECTION=201.html (18.02.2006; 20 Uhr)

(2002b): Memory of the World: General Guidelines. Doc. CII-95/WS-11rev. Paris 
UNESCO (2002c): Allgemeine Erklärung zur kulturellen Vielfalt. In: UNESCO heute. Zeitschrift der Deutschen UNESCO-Kommission 1-2; http://www.unesco.de/pdf/deklaration_kulturelle _vielfalt.pdf (21.03.2006; 19 Uhr)

---- (2003a): Abkommen zum Schutz des immateriellen Kulturerbes. UNESCO http://www.unesco.ch/pdf/konvention_immaterielles_kulturerbe.pdf (18.02.2006; 21 Uhr)

(2003b): Meisterwerke traditioneller Weltkultur. UNESCO heute 11-12; http://www.unesco-heute.de/1103/meisterwerke.htm

(2003c): Seconde Proclamation of Masterpieces of the Oral and Intangible Heritage of Humanity. UNESCO http://portal.unesco.org/culture/en/ev.phpURL_ID=2226\&URL_DO=DO_TOPIC\&URL_SECTION=201.html

(2004): Guidelines Living Human Treasures. http://portal.unesco.org/culture/ en/file_download.php/1422690320114549c199903cf8ba93f9 Guidelines_Iht.pdf (23.02.2006; 19 Uhr)

(2005a): Welterbeliste. http://whc.unesco.org/pg.cfm?cid=31 (24.01.2006; 21 Uhr)

(2005b): Liste der Vertragsstaaten der Haag Konvention von 1954. UNESCO http://erc.unesco.org/cp/convention.asp?KO=13637\&language=E (11.02.2006; $18 \mathrm{Uhr})$

(2005c): Liste der Mitgliedsstaaten der Welterbekonvention. UNESCO http://whc.unesco.org/pg.cfm?cid=246 (14.02.2006; 19 Uhr)

(2005d): Operational Guidelines for the Implementation of the World Heritage Convention. UNESCO http://whc.unesco.org/archive/opguide05-en.pdf (18.02.2006; $21 \mathrm{Uhr}$ )

(2005e): Proclaimed Masterpieces 2005. http://www.unesco.org/culture/intangible-heritage/ (23.02.2006; $20 \mathrm{Uhr})$

(2005f): Convention on the Protection and Promotion of the Diversity of Cultural Expressions. http://unesdoc.unesco.org/images/0014/001429/142919e.pdf (24.02.2006; 22 Uhr)

---- (2005g): Third Proclamation of Masterpieces of the Oral and Intangible Heritage of Humanity. UNESCO http://portal.unesco.org/culture/en/ev.phpURL_ID=2226\&URL_DO=DO_TOPIC\&URL_SECTION=201.html 
UNESCO (2006): Liste der Mitgliedsstaaten der UNESCO. http://erc.unesco.org/cp/ MSList_alpha.asp?lg=E (27.01.2006; 16 Uhr)

UNIDROIT (1995): Convention on Stolen or Illegally Exported Cultural Objects. Rome

U.S. Information Agency (1992): Curbing Illicit Trade in Cultural Property: U.S. Assistance under the Convention on Cultural Property Implementation Act. Washington D.C.

Valderama, F. (1995): A History of UNESCO. Paris: UNESCO

Van Hasselt, J. (1996): Die UNESCO-Strategie für Kultur und Entwicklung bis zum Pérez-de-Cuéllar Bericht. In: Hüfner, K.; W. Reuther (Hg.): UNESCO-Handbuch. Neuwied [u.a.]: Luchterhand

Van Velzen, D.T. (1996): The World of Tuscan Tomb Robbers: Living with the Local Community and the Ancestors. In: International Journal of Cultural Property 5 (1): 111-126

Van Zanten, W. (2004): Constructing New Terminology for Intangible Cultural Heritage. In: Museum International 56(1-2):36-43

Viehoff, R.; R.T. Segers (Hg.)(1999): Kultur, Identität, Europa. Über die Schwierigkeiten und Möglichkeiten einer Konstruktion. Frankfurt/ Main: Suhrkamp

Virilio, P. (1986): Ästhetik des Verschwindens. Berlin: Merve-Verlag

Wald, P. (1969): Die Vereinigte Arabische Republik. Hannover: Verlag für Literatur und Zeitgeschehen

Wallerstein, I. (1986): Das moderne Weltsystem. Kapitalistische Landwirtschaft und die Entstehung der europäischen Weltwirtschaft im 16. Jahrhundert. Frankfurt/ Main: Syndikat

Ward, R.G. (ed.)(1995): Land, custom and practice in the South Pacific. Cambridge: Cambridge Uni. Press

Watson, P. (1997): Sotheby's Inside Story. London: Bloomsbury

Watson, S. (2003): Valuing world heritage. North Mankato: Smart Apple Media

Weiner, A.B. (1992): Inalienable Possession: The paradox of keeping-while-giving. Berkeley: Uni. of California Press 
Weigel, S.; B. Jussen (2005): Erbe, Erbschaft, Vererbung. Überlieferungskonzepte zwischen Natur und Kultur im historischen Wandel. Bielefeld: Volkswagen Stiftung; http://www.erbschaftsforschung.de/projekt_enter.html; (25.04.2006; 18 Uhr)

Welsh, P.H. (1997): The Power of Possession: The Case against Property. In: Museum Anthropology 21:12-18

Wermke, M. et al (Hg.)(2001): Herkunftswörterbuch. Etymologie der deutschen Sprache. Der Duden in zwölf Bänden. 3. Aufl. Mannheim [u.a.]: Dudenverlag

Willems, H.; A. Hahn (Hg.)(1999): Identität und Moderne. Frankfurt/Main: Suhrkamp

Wilson, H.T.; N.J. Parezo (1992): The Role of Museum in Preserving the Anthropological Record. In: Silverman, S.; N.J. Parezo (eds.): Preserving the Anthropological Record. 62-72; New York: Wenner-Gren Foundation

Yeingst, J. (1987): INTERPOL's Stolen Art Program. In: Journal of Field Archaeology 14: 222-223 


\section{Abkürzungsverzeichnis}

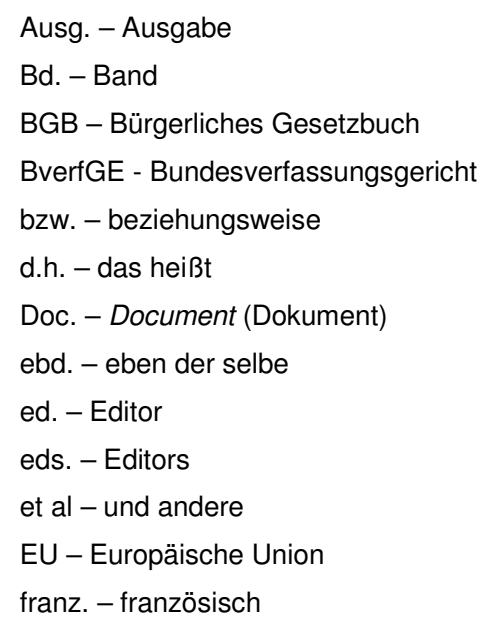




\section{Eidesstattliche Versicherung}

„Ich versichere hiermit eidesstattlich, dass ich die Arbeit selbständig und nur mit den angegebenen Quellen und Hilfsmittel angefertigt habe.“

Göttingen der 03.07.2006

Unterschrift des Verfassers:

Frank-André Weigelt 